\title{
A Wind-tunnel Investigation of an Ultra-light Wing and Ultra-light Aircraft
}

\author{
by \\ Wael Khaddage \\ A thesis submitted to
the Faculty of Graduate and Postdoctoral Affairs \\ in partial fulfilment of \\ the requirements for the degree of \\ Master of Applied Science \\ in \\ Aerospace Engineering
}

Ottawa-Carleton Institute for Mechanical and Aerospace Engineering

Department of Mechanical and Aerospace Engineering

Carleton University

Ottawa, Ontario, Canada

April 2017

Copyright (C)

2017 - Wael Khaddage 


\section{Abstract}

A wind-tunnel investigation was undertaken on a scaled down rigid model of both an ultra-light wing and an ultra-light aircraft. The results of the ultra-light wing were compared to an existing computation fluid dynamics (CFD) simulation for validation purposes. The comparisons indicated that the results of the simulation did not agree with those from experimentation; it is most notably observed in the drag coefficient results as they are clearly erroneous and further work is required on developing a validated simulation. The basic performance characteristics along with a longitudinal and lateral static stability analysis were completed. It was observed that the aircraft's drag polar does not conform to a classic parabolic shape commonly used to describe conventional fixed-wing aircraft; the suspended fuselage was found to have a dominant effect on the shape of the drag polar when compared to the wing-only experiments. Furthermore, the aircraft was found to be statically stable in pitch and statically unstable in yaw. The pitch stiffness, $c_{M_{\alpha}}$, was determined to be $-8.33 \mathrm{rad}^{-1}$. The weathercock stability derivative, $c_{N_{\beta}}$, was determined to be $-0.0274 \mathrm{rad}^{-1}$ implying directional instability. 


\section{Acknowledgments}

I would like to express my sincerest gratitude and appreciation to my supervisor Professor Jeremy Laliberté for his support, guidance, and patience throughout my graduate studies. I would also like to thank Professor Laliberté for giving me an opportunity to undertake research in a field of interest while gaining valuable experience.

I would like to acknowledge my colleague Darren Penley for encouraging me to pursue a Master's Degree and for all of his help and encouragement over the past two years - thank you, your support was greatly appreciated.

I am grateful to Romaeris Corporation for their financial support which allowed me to pursue my research without financial burden.

I would like to recognize Alan Redmond for his advice and his professionalism from the very beginning of my research. I was able to get a real taste of industry experience under your supervision.

Lastly, I would like to thank my family for their care, understanding, and unconditional support throughout my studies. I could not have done this without you. 


\section{Table of Contents}

Abstract

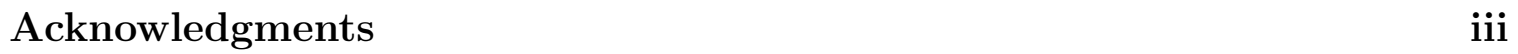

Table of Contents iv

List of Tables vii

List of Figures viii

List of Acronyms $\quad$ xi

List of Symbols $\quad$ xii

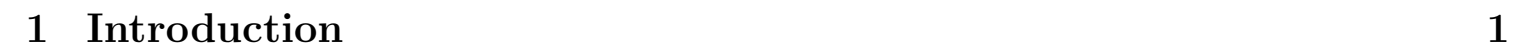

1.1 Motivation . . . . . . . . . . . . . . . . . . . . . 1

1.2 Objectives . . . . . . . . . . . . . . . . . . . 2

1.3 Scope . . . . . . . . . . . . . . . . . . . . 2

1.4 Contributions . . . . . . . . . . . . . . . . . 3

\begin{tabular}{|lll}
\hline 2 & Literature Review & 4 \\
\hline
\end{tabular}

2.1 Ultra-light Aircraft . . . . . . . . . . . . . . . . . . 4

$2.1 .1 \quad$ Ultra-light Trike . . . . . . . . . . . . . . . 6

$2.1 .2 \quad$ Ultra-light Trike Aerodynamics . . . . . . . . . . . . . . . . . 8 
2.1 .3 Modeling an Ultra-light Trike . . . . . . . . . . . . . . . . 12

2.2 Static Stability $\ldots \ldots \ldots \ldots \ldots$

$2.2 .1 \quad$ Longitudinal Static Stability Derivatives . . . . . . . . . . . . 24

2.2 .2 Lateral Static Stability Derivatives . . . . . . . . . . . . . 25

2.3 Similarity Parameters . . . . . . . . . . . . . . . . . . . . . 25

2.3 .1 Geometric Similarity . . . . . . . . . . . . . 26

2.3 .2 Kinematic Similarity . . . . . . . . . . . . . 26

2.3 .3 Dynamic Similarity . . . . . . . . . . . . . . . . . . . 27

2.4 Parameter Estimation $\ldots \ldots \ldots$. . . . . . . . . . . . . . . . . 28

$\begin{array}{|ll|}3 & \text { Experimental Setup and Procedures }\end{array}$

3.1 Overview . . . . . . . . . . . . . . . . . . . . . . . . 30

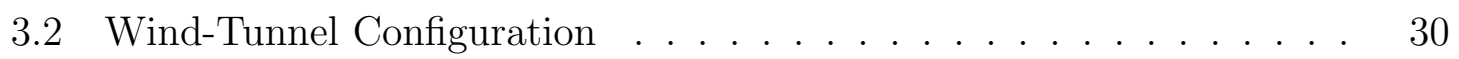

3.3 Model Design and Manufacture . . . . . . . . . . . . . . . . . . 32

$3.3 .1 \quad$ Ultra-light Wing . . . . . . . . . . . . . . . . . . . . . . . 32

3.3 .2 Ultra-light Wing and Fuselage . . . . . . . . . . . . . . . . . . 34

3.3 .3 Blockage Effects . . . . . . . . . . . . . . . . . . . 37

3.3 .4 Trip Strip . . . . . . . . . . . . . . . . . . . . . . . . . . . 39

3.4 Model Support . . . . . . . . . . . . . . . . . . . . . . . . . . . . . . 43

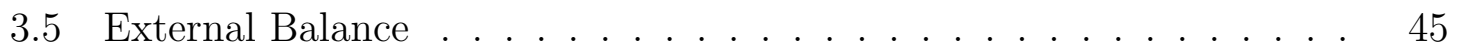

3.5.1 Calibration of Load Cells . . . . . . . . . . . . . . . . . . . . 47

3.6 Instrumentation $\ldots \ldots \ldots \ldots$. . . . . . . . . . . . . . . . . . . . . . . . . . 48

3.6.1 $\quad$ Inclinometer . . . . . . . . . . . . . . . . . . . . . . . . . . . . . . . . 48

3.6 .2 Thermometer . . . . . . . . . . . . . . . . . . . 49

3.6 .3 Pressure Measurement Device . . . . . . . . . . . . . . . . . 50

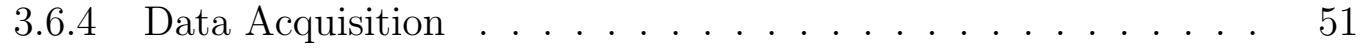

3.6.5 Wind-Tunnel Commissioning Experiments . . . . . . . . . . 52 
3.7 Experimental Uncertainties $\ldots \ldots \ldots \ldots$

3.8 Test Matrix . . . . . . . . . . . . . . . . . . . . . . . 57

3.9 Experimental Procedure: Wing-Only $\ldots \ldots \ldots \ldots \ldots$

3.9 .1 Test Case $1 \ldots \ldots \ldots \ldots$

3.10 Experimental Procedure: Ultra-light Model . . . . . . . . . . . 60

3.10 .1 Test Case $1 \ldots \ldots \ldots \ldots$

3.10 .2 Test Case $2 \ldots \ldots \ldots \ldots \ldots$

3.11 Data Reduction . . . . . . . . . . . . . . . . . 66

\begin{tabular}{|lll|}
\hline 4 & Experimental Results and Discussions & 67
\end{tabular}

4.1 Repeatability of the Experiment . . . . . . . . . . . . 67

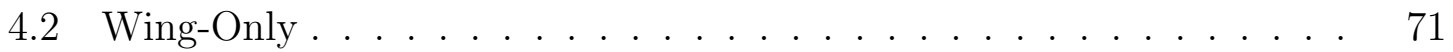

$4.3 \quad$ Ultra-light Model . . . . . . . . . . . . . . . . . . . . . . . . . . . 81

4.3 .1 Test Case $1 \ldots \ldots \ldots$

4.3 .2 Test Case $2 \ldots \ldots \ldots \ldots$

5 Conclusions, Limitations, and Recommendations for Future Work 102

5.1 Conclusions $\ldots \ldots \ldots \ldots \ldots$

5.2 Limitations $\ldots \ldots \ldots \ldots \ldots \ldots$

5.3 Recommendations . . . . . . . . . . . . . . . . . . . . 104

\begin{tabular}{ll}
\hline References & 106
\end{tabular}

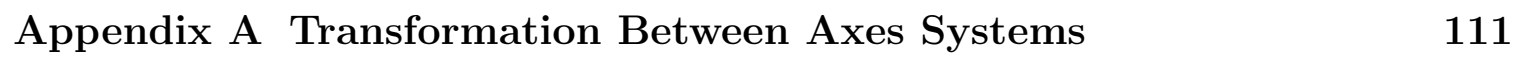

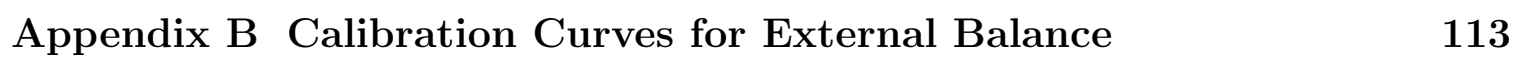

Appendix C Ultra-light Trike Reynolds Number in Various Flight Con-

$\begin{array}{ll}\text { ditions } & 117\end{array}$




\section{List of Tables}

2.1 Nondimensional force and moment coefficients for conventional aircraft. 13

$2.2 \quad$ Hang glider forces and moments expressed about the centre of gravity. 15

3.1 Test section specifications $\ldots \ldots \ldots \ldots$

$3.2 \quad$ Key data for the full scale PROFI TL 14 ultra-light wing. . . . . . . 32

$3.3 \quad$ Fluke-179 True RMS Digital Multimeter specifications. . . . . . . . . 51

3.4 Quantification of the bias error in the instrumentation. . . . . . . . 56

$3.5 \quad$ Test matrix for the wing-only experiments. . . . . . . . . . . 58

$3.6 \quad$ Test matrix for ultra-light model experiments. . . . . . . . . . . 58

4.1 Computational and experimental lift curve slope results . . . . . . . 75

$4.2 \quad$ Pitch stiffness slope, zero angle of attack pitching moment, and trim angle of attack for wing-only experiments. . . . . . . . . . . . 79

$4.3 \quad$ Pitch stiffness slope, zero angle of attack pitching moment, and trim angle of attack: ultra-light model. . . . . . . . . . . . . . . . . . 89

$\begin{array}{llll}4.4 & \text { Summary of the results for the static stability derivatives } c_{N_{\beta}} \text { and } c_{Y_{\beta}} & 101\end{array}$

C.1 Typical Reynolds Number Range for Ultra-light Trikes . . . . . . . . 117 


\section{List of Figures}

2.1 Basic ultra-light aeroplane . . . . . . . . . . . . . . . . . . 5

2.2 Advanced ultra-light aeroplane. . . . . . . . . . . . . . . 5

2.3 Ultra-light trike with a single surface wing. . . . . . . . . . . . . . . . 7

$2.4 \quad$ Ultra-light trike with an airfoil-shaped wing. . . . . . . . . . . . . . . 7

2.5 Typical rigid wing airfoil compared to an ultra-light airfoil. . . . . . . 9

$2.6 \quad$ Lift generation at the wing root and wing tip for ultra-light wings. . . 10

2.7 Lift curve at the minimum controlled airspeed for an ultra-light aircraft. 11

2.8 Conventions for aircraft body axes and stability axes. . . . . . . . . . 12

2.9 Cook and Spottiswoode hang glider model. . . . . . . . . . . . . . . 15

2.10 One-body simplification of the Cook and Spottiswoode hang glider model. . . . . . . . . . . . . . . . . . . . . . 16

2.11 Ochi two-body hang glider model. . . . . . . . . . . . . . . . . 18

2.12 Pitch stiffness condition for longitudinal static stability. . . . . . . . . 19

2.13 Requirement for yaw stability in a sideslip. . . . . . . . . . . . . . . . 21

2.14 Requirement for roll stability in a sideslip. . . . . . . . . . . . . . . . 21

2.15 Restoring yaw moment generated by wing sweep when the aircraft is in a sideslip. . . . . . . . . . . . . . . . . . . . . . . . . . . . . 22

2.16 Restoring roll moment when the aircraft is in a sideslip. . . . . . . . . 23

$3.1 \quad$ Schematic of the Carleton University closed-circuit wind-tunnel. . . . 31

3.2 Modification to the scaled-down ultra-light wing. . . . . . . . . . . . 33 
$3.3 \quad$ CAD model of the PROFI TL 14 ultra-light wing. . . . . . . . . 34

3.4 CAD model of the PROFI TL 14 ultra-light wing with the trailing edge at the root modified. . . . . . . . . . . . . . . . . . 34

$3.5 \quad$ Preliminary design of the fuselage for the ultra-light aircraft. . . . . . 35

$3.6 \quad$ CAD model of the aircraft assembly. . . . . . . . . . . . . 36

$3.7 \quad$ Representation of the wake blockage effects within the test section. . 38

3.8 Representation of a laminar and transitional boundary layers. . . . . 40

$3.9 \quad$ Change in drag coefficient with respect to a change in grit size. . . . . 41

3.10 Ultra-light wing model with the addition of a 3-dimensional trip strip. 42

3.11 Ultra-light wing and sphere configuration . . . . . . . . . . . 44

3.12 Sphere used for wing-only experiments . . . . . . . . . . . . 44

3.13 Wind-tunnel external 3-component mechanical balance mounted above the test section. . . . . . . . . . . . . . . . . . . 46

3.14 Inclinometer on the external balance. . . . . . . . . . . . . . 49

3.15 Thermometer and temperature gauge in the wind-tunnel contraction. 50

3.16 Schematic of the wind-tunnel contraction. . . . . . . . . . . . 51

3.17 Velocity correlation between the pitot-static probe and the contraction pressure difference. . . . . . . . . . . . . . . . . 53

3.18 Manometer height correlation between the pitot-static probe and the contraction pressure difference. . . . . . . . . . . . . . . 54

3.19 Velocity profile along the height of the test section. . . . . . . . 55

3.20 Representation of Test Case 1 with the model connected to external balance. . . . . . . . . . . . . . . . . . . 61

3.21 Representation of Test Case 1 with respect to the wind-tunnel axes. . 62

3.22 Representation of Test Case 2 with the model connected to external balance. . . . . . . . . . . . . . . . . . . 64

3.23 Representation of Test Case 2 with respect to the wind-tunnel axes. . 65 
4.1 Repeatability test: $c_{L}$ vs. $\alpha . \ldots \ldots$. . . . . . . . . . 68

4.2 Repeatability test: $c_{D}$ vs. $\alpha . \ldots \ldots \ldots$. . . . . . . . 69

4.3 Repeatability test: $c_{M}$ vs. $\alpha . \ldots . \ldots . \ldots . \ldots 70$

4.4 Wing-only test $-c_{L}$ vs. $\alpha$. . . . . . . . . . . . . 72

4.5 Wing-only test compared to the CFD results $-c_{L}$ vs. $\alpha . \ldots . . . . \quad 74$

4.6 Wing-only test $-c_{D}$ vs. $\alpha . \ldots \ldots \ldots$. . . . . . . . 76

4.7 Wing-only test compared to the CFD results $-c_{D}$ vs. $\alpha . \quad \ldots . . .78$

4.8 Wing-only test $-c_{M}$ vs. $\alpha$. . . . . . . . . . . . . . . . . 80

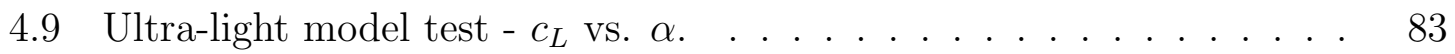

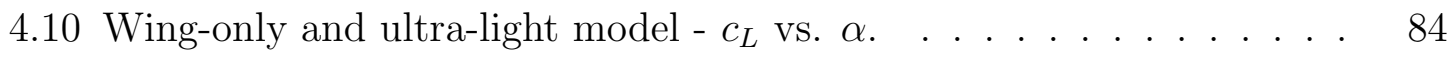

4.11 Drag polar of the ultra-light model test $-c_{L}$ vs. $c_{D} \ldots \ldots$. . . . . 86

4.12 Drag Polar of the ultra-light model and ultra-light wing $-c_{L}$ vs. $c_{D} . \quad 87$

4.13 Ultra-light model test $-c_{M}$ vs. $\alpha$. . . . . . . . . . . . . . . . 90

4.14 Ultra-light model test $-c_{N}$ vs. $\beta$. . . . . . . . . . . . . . . 92

4.15 Ultra-light model test $-c_{Y}$ vs. $\beta$. . . . . . . . . . . . . . . 94

4.16 Ultra-light model test $-c_{N}$ vs. $\beta$ (corrected). . . . . . . . . . . . 96

4.17 Ultra-light model test $-c_{Y}$ vs. $\beta$ (corrected). . . . . . . . . . . . . 97

4.18 Ultra-light model test $-c_{Y}$ vs. $\beta$ (measured and calculated). . . . . . 99

4.19 Ultra-light model test $-c_{Y}$ vs. $\beta$ (removed flow-normal load cell measurements). . . . . . . . . . . . . . . . . . . . . . 100

A.1 Ultra-light trike body axes system. . . . . . . . . . . . . . . . . . . . 112

B.1 Axial load cell calibration curve. . . . . . . . . . . . . . . . . . . . . . 114

B.2 Normal load cell calibration curve. . . . . . . . . . . . . . . . . . . . 115

B.3 Pivot load cell calibration curve. . . . . . . . . . . . . . . . . . . 116 


\section{List of Acronyms}

\begin{tabular}{ll}
\hline Acronyms & Definition \\
\hline AR & Aspect ratio \\
ABS & Acrylonitrile butadiene styrene \\
CAD & Computer-aided design \\
CFD & Computational fluid dynamics \\
IAS & Indicated airspeed \\
MTOW & Maximum takeoff weight \\
WSC & Weight-shift control \\
\hline
\end{tabular}




\section{List of Symbols}

\begin{tabular}{ll} 
Symbols & Definition \\
\hline$\alpha$ & angle of attack \\
$\beta$ & sideslip angle \\
$c_{\mathscr{L}}$ & roll moment coefficient \\
$c_{M}$ & pitching moment coefficient \\
$c_{N}$ & yaw moment coefficient \\
$e$ & Oswald efficiency factor \\
$g$ & gravitational acceleration $\left(9.81 \mathrm{~m} / \mathrm{s}^{2}\right)$ \\
$h$ & height of trip strip (in) \\
$K$ & conditions at the top of roughness particle \\
$l$ & characteristic length $(\mathrm{m})$ \\
$\mathscr{L}$ & roll moment $(\mathrm{N} \cdot \mathrm{m})$ \\
$\mu$ & dynamic viscosity $(\mathrm{kg} / \mathrm{m} \cdot \mathrm{s})$ \\
$M$ & pitch moment $(\mathrm{N} \cdot \mathrm{m})$
\end{tabular}




$\begin{array}{ll}M_{\infty} & \text { freestream Mach number } \\ N & \text { yaw moment }(\mathrm{N} \cdot \mathrm{m}) \\ p_{\text {atm }} & \text { atmospheric pressure }(\mathrm{Pa}) \\ \phi & \text { bank angle } \\ \rho & \text { density }\left(\mathrm{kg} / \mathrm{m}^{3}\right) \\ R_{\text {air }} & \text { specific gas constant for dry air }(287 \mathrm{~J} / \mathrm{kg} \cdot \mathrm{K}) \\ R e & \text { Reynolds number } \\ S & \text { projected wing area }\left(\mathrm{m}^{2}\right) \\ T & \text { temperature }(\mathrm{K}) \\ V & \text { freestream velocity }(\mathrm{m} / \mathrm{s})\end{array}$




\section{Chapter 1}

\section{Introduction}

\subsection{Motivation}

For decades, ultra-light trikes have be flown globally for recreational purposes. Ultra-light trikes are powered flexible wing manned-aircraft with a suspended carriage. Very few researchers have attempted to mathematically model such aircraft and most research to date has been empirical or anecdotal. The lack of interest in ultra-light trikes for humanitarian or commercial purposes is regrettable due to the aircraft's unique capabilities. Not only are these ultra-light aircraft low cost but they are relatively light and have an impressive payload capacity. Furthermore, the fuel consumption of these types of aircraft are low compared to larger aircraft.

Ultra-light trikes are weight-shift control (WSC) aircraft. Therefore, the stability and manoeuvrability of these aircraft are dictated by the pilot's physical ability. For high endurance flights, pilot fatigue becomes a dangerous issue. Developing a representative model of an ultra-light aircraft can provide a more in-depth understanding of its expected behaviour which will in turn increase its safety and efficiency. Furthermore, if a flight controller is to be developed in order to alleviate the strain on the pilot by making the necessary adjustments to remain in steady-level flight, it could 
create opportunity for various applications. These aircraft could deliver supplies and time-sensitive products to isolated communities around the world that may otherwise be unaccessible by conventional aircraft.

\subsection{Objectives}

This research aims to investigates the basic aerodynamic characteristics of ultralight aircraft using wind-tunnel experimentation. The work consisted of two major objectives. The first objective is to determine the lift and drag coefficients of an existing ultra-light wing in isolation as its angle of attack changes. The results from these experiments will be used to validate an existing computational fluid dynamics (CFD) simulation undertaken by a colleague. The second objective is to extract static stability derivatives of an ultra-light aircraft as a whole in order to determine the degree of static stability of the aircraft.

\subsection{Scope}

The research presented in this thesis was proposed by the Romaeris Corporation. The original scope of the work was to attempt to implement active winglets on an ultra-light wing in order to test their effectiveness on stability, control, and performance. It quickly became clear that the necessity to aerodynamically characterize the ultra-light wing and fuselage would have to be a stepping stone prior to the winglet analysis. Consequently, the focus of the research shifted to wind-tunnel experiments on ultra-light aircraft. The tests were to be conducted on a rigid scale model of an actual ultra-light wing and with an accompanying preliminary design of a fuselage. In real world application of ultra-light aircraft, aeroelastic effects have significant impact on the aircraft's behaviour due to the wing's flexible nature; 
modelling such effects makes the problem difficult due to nonlinearities. Therefore, a rigid body approach was chosen in order to develop baseline results from which future experiments may be compared and validated.

The research presented in this thesis is also to be used as a guideline for the continuation of wind-tunnel experiments on ultra-light aircraft. The results obtained during experimentation are a first pass at static stability analysis of ultra-light aircraft and future work should build off the methods outlined in this thesis to ensure fluidity in the advancement of the research.

\subsection{Contributions}

Historically, the research of ultra-light trikes is limited; the research presented in this thesis lays the groundwork for understanding the aircraft's expected static behaviour. The work described in the thesis will also contribute to the development of a flight

controller for and ultra-light trike aircraft. This will open up the opportunity for practical applications of these type of aircraft. Due to their relatively low capital and operational costs, the use of WSC aircraft as a method of transportation for humanitarian initiatives could have a positive impact worldwide. 


\section{Chapter 2}

\section{Literature Review}

\subsection{Ultra-light Aircraft}

The definition of an ultra-light aircraft varies from country to country. Two categories of ultra-light aircraft have been defined by the Canadian Aviation Regulations: basic ultra-light aeroplane and advanced ultra-light aeroplane (Figures 2.1 and 2.2. . Any aircraft that meets any of the three definitions listed below is designated as a basic ultra-light aeroplane [1].

1. An aircraft with one seat having a maximum takeoff weight (MTOW) of up to $165 \mathrm{~kg}$. The wing area $(S)$ can be no less than $10 \mathrm{~m}^{2}$ and no greater than the MTOW minus 15 divided by 10 .

2. An instructional aircraft with two seats having a MTOW of up to $195 \mathrm{~kg}$. The wing area can be no less than $10 \mathrm{~m}^{2}$ and the wing loading $\left(\frac{M T O W}{S}\right)$ can be no greater than $25 \mathrm{~kg} / \mathrm{m}^{2}$.

3. An aircraft with up to two seats having a MTOW of $544 \mathrm{~kg}$ and a landing stall speed no greater than $72 \mathrm{~km} / \mathrm{h}$ (39 knots) indicated airspeed (IAS). The indicated airspeed refers to the uncorrected reading from the airspeed indicator measured by a pitot-static probe. Factors such as temperature, density, and instrumentation error are not considered when reading the IAS [2]. 


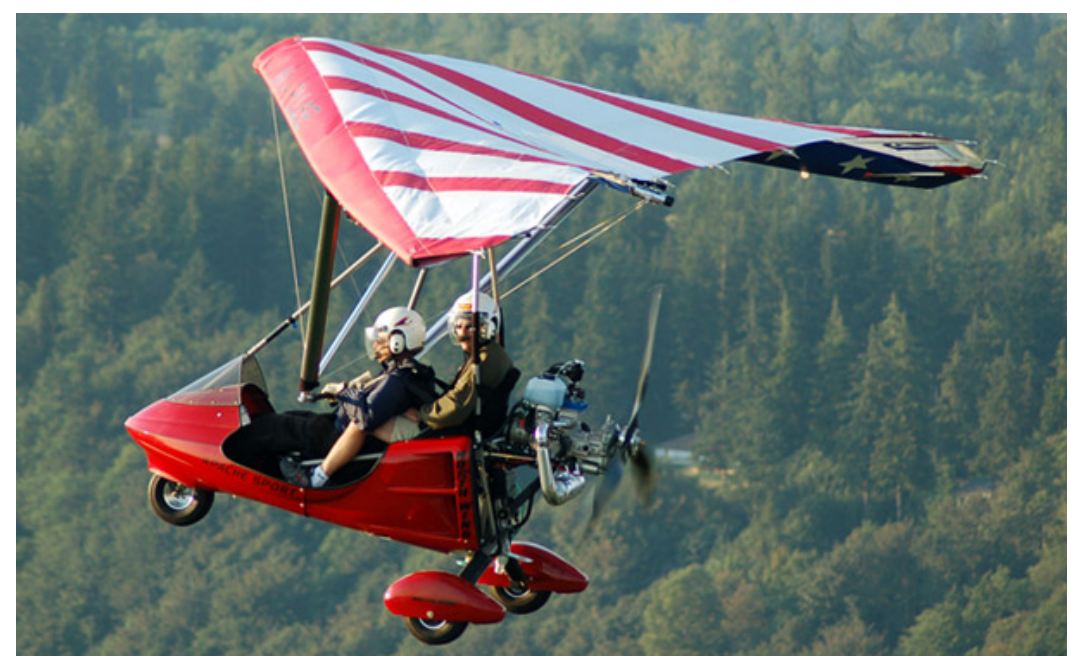

Figure 2.1: Basic ultra-light aeroplane 3].

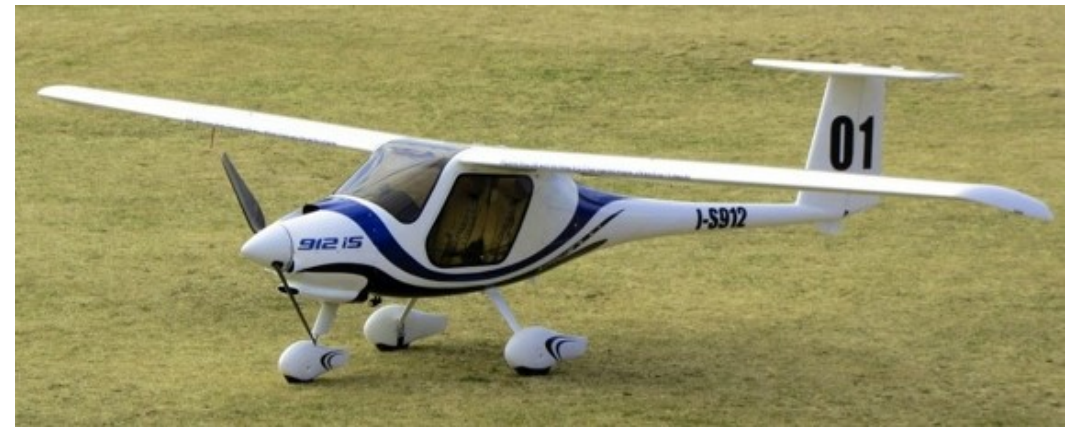

Figure 2.2: Advanced ultra-light aeroplane 4.

Any ultra-light aircraft that does not fall under the definition of a basic ultra-light aeroplane may potentially be classed as an advanced ultra-light. As per Transport Canada, an aircraft must adhere to the design, structural, performance, and power requirements listed in the Design Standards for Advanced Ultra-light Aeroplanes in order for it to be considered an advanced ultra-light [1,5]. This manual was developed by the Light Aircraft Manufacturers Association of Canada.

In brief, the manual defines an advanced ultra-light as a propeller-driven aircraft that may carry up to two persons. If the aircraft is designed for a single person, its MTOW is limited to $350 \mathrm{~kg}$. Alternatively, if the aircraft is designed for two persons, 
its MTOW is limited to $560 \mathrm{~kg}$. The landing stall speed can be no greater than an of $72 \mathrm{~km} / \mathrm{h}$ (39 knots) IAS regardless of the capacity [5]. Powered trikes, gliders, and parachutes are not included in the category of advanced ultra-light aeroplanes.

These classifications of ultra-light aeroplanes are exclusive to Canada. In the United States, they fall under the broad definition of Light Sport Aircraft [6]. In the United Kingdom, they are defined as microlight aircraft [7].

\subsubsection{Ultra-light Trike}

An ultra-light trike is a basic ultra-light aircraft comprised of a fuselage (or carriage) suspended from a wing. The fuselage may seat up to two persons and may be equipped with wheels or skis. The fuselage may also be an inflatable boat used for takeoff and landing on water [6]. An engine and propeller are located at the rear of the fuselage. The propeller size is dependent on the clearance between the wing and the fuselage.

Earlier versions of ultra-light trike wings were single surface wings similar to hang gliders. Numerous rods were attached to a triangular fabric cloth in order to aerodynamically shape it. An example of an ultra-light trike with a single surface wing can be seen in Figure 2.3 [6]. Current wings are made up of a fabric cloth draped over airfoil-shaped ribs. Due to the fabric wing's deformable nature, the wing is significantly influenced by aeroelastic effects. Aerodynamic loads will induce bending and twisting on the wing. The magnitude of these deformations are dependent on the airspeed and attitude. This, in turn, leads to nonlinear aerodynamic effects [8]. Figure 2.4 illustrates an example of a modern ultra-light [6]. 


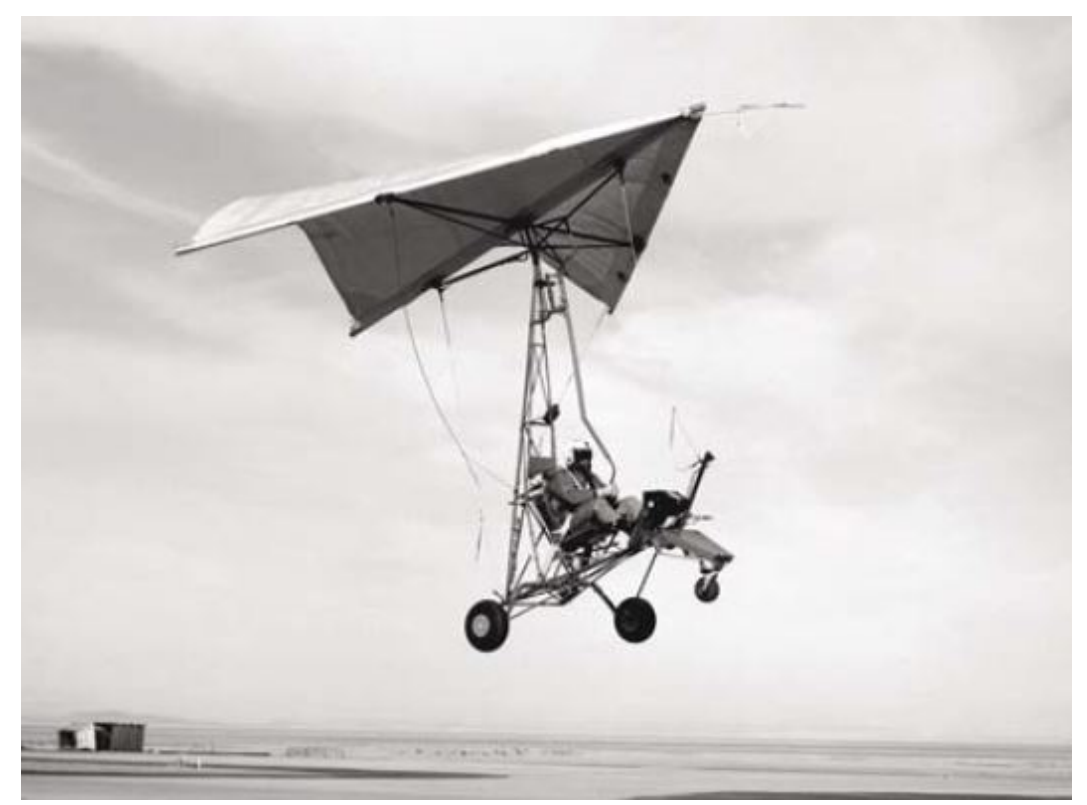

Figure 2.3: Ultra-light trike with single surface wing [6].

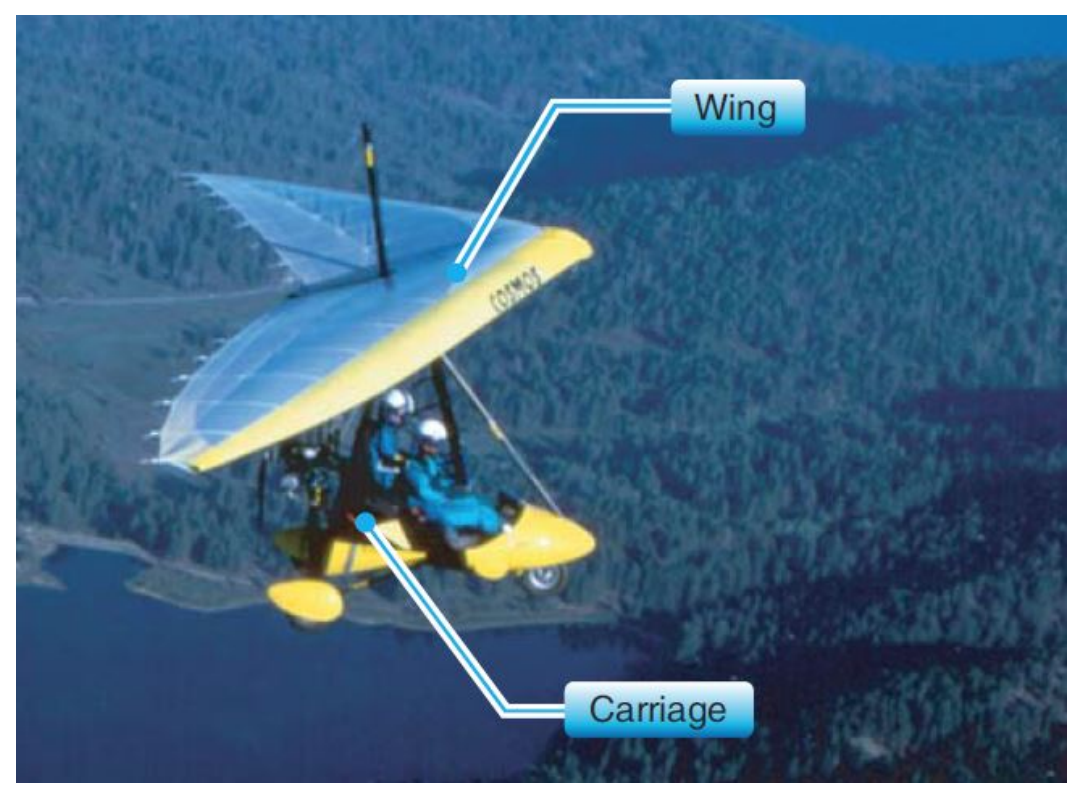

Figure 2.4: Ultra-light trike with an airfoil-shaped wing [6].

Ultra-light trikes are robust aircraft making them versatile aircraft capable of undertaking various missions. They are capable of takeoff and landing on different types of terrain and in various weather conditions. In the case of emergency landings 
caused by engine failure, the pilot has the ability to control the aircraft's attitude and sink rate to a certain extent making it possible to minimize damage to the aircraft or the likelihood of injury to the occupants [6].

\subsubsection{Ultra-light Trike Aerodynamics}

The airfoil shape of an ultra-light trike differs from that of a conventional aircraft. This type of airfoil may also be referred to as a WSC airfoil [6]. Its camber is significantly less pronounced and its chordwise thickness reduction is essentially linear downstream of the high point (or point of maximum thickness). Furthermore, the high point on the airfoil is typically located closer to the leading edge compared to airfoils found on conventional aircraft [6]. This comparison is illustrated in Figure 2.5 [6]. The difference in high point location increases the longitudinal stability due to the nature of the airfoil.

The subsonic aerodynamic centre of an airfoil is approximately located at the quarter-chord location from the leading edge [9,10]. The pitching moment about the aerodynamic centre will remain constant regardless of the lift force generated by the airfoil. In other words, it is not a function of the total lift or angle of attack. The centre of pressure is the position along the chord at which the integrated lift can be represented by a single lift force; the pitching moment about this position is zero [10]. The location of the centre of pressure varies as the angle of attack and speed change making it unsuitable for aerodynamic load analysis on an airfoil. On the ultra-light airfoil, the centre of pressure is located in the proximity of the high point whereas it lies behind the quarter-chord location for conventional cambered airfoils at low subsonic speeds [6,11]. Conventional cambered airfoils generally have a negative (nose down) pitching moment due to the pressure distribution. Conversely, an ultra-light airfoil is designed to generate either a neutral or positive (nose up) 
pitching moment [6]. This airfoil shape is strategically selected to be less strenuous on the pilot as it is more difficult to counteract a downwards pitching moment.

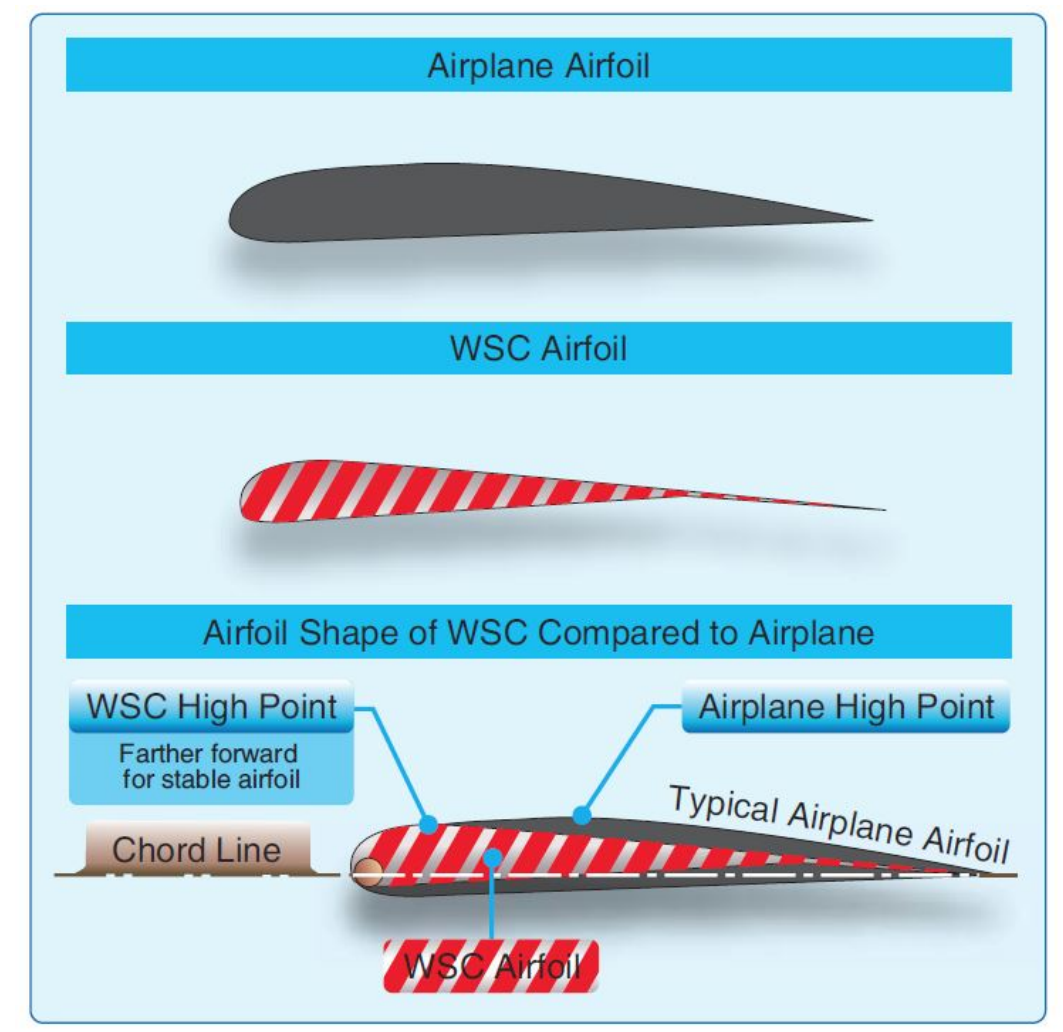

Figure 2.5: Typical rigid wing airfoil compared to an ultra-light airfoil [6].

Longitudinal stability in ultra-light trikes is achieved as a result of wing twist varying as a function of aerodynamic loading. Under aerodynamic loads, the wing undergoes significant twisting. This twisting motion reduces the local angle of attack in the spanwise direction from root to tip. Therefore, the angle of attack at the wing tip is smaller than the angle of attack at the root [6]. While conventional aircraft use the tail to generate downwards lifting forces, an ultra-light's wing tip deflection acts as a passive control surface for longitudinal stability. The wing's relatively large sweep angle places the wing tip aft of the aircraft's centre of gravity. In doing so, the total lift is distributed in a manner in which the lift fore and aft of the centre of gravity generate pitching moments in opposite directions. When the aircraft is in a 
trim state, the total lift acts in line with the centre of gravity. This is illustrated in the part $A$ of Figure 2.6 [.

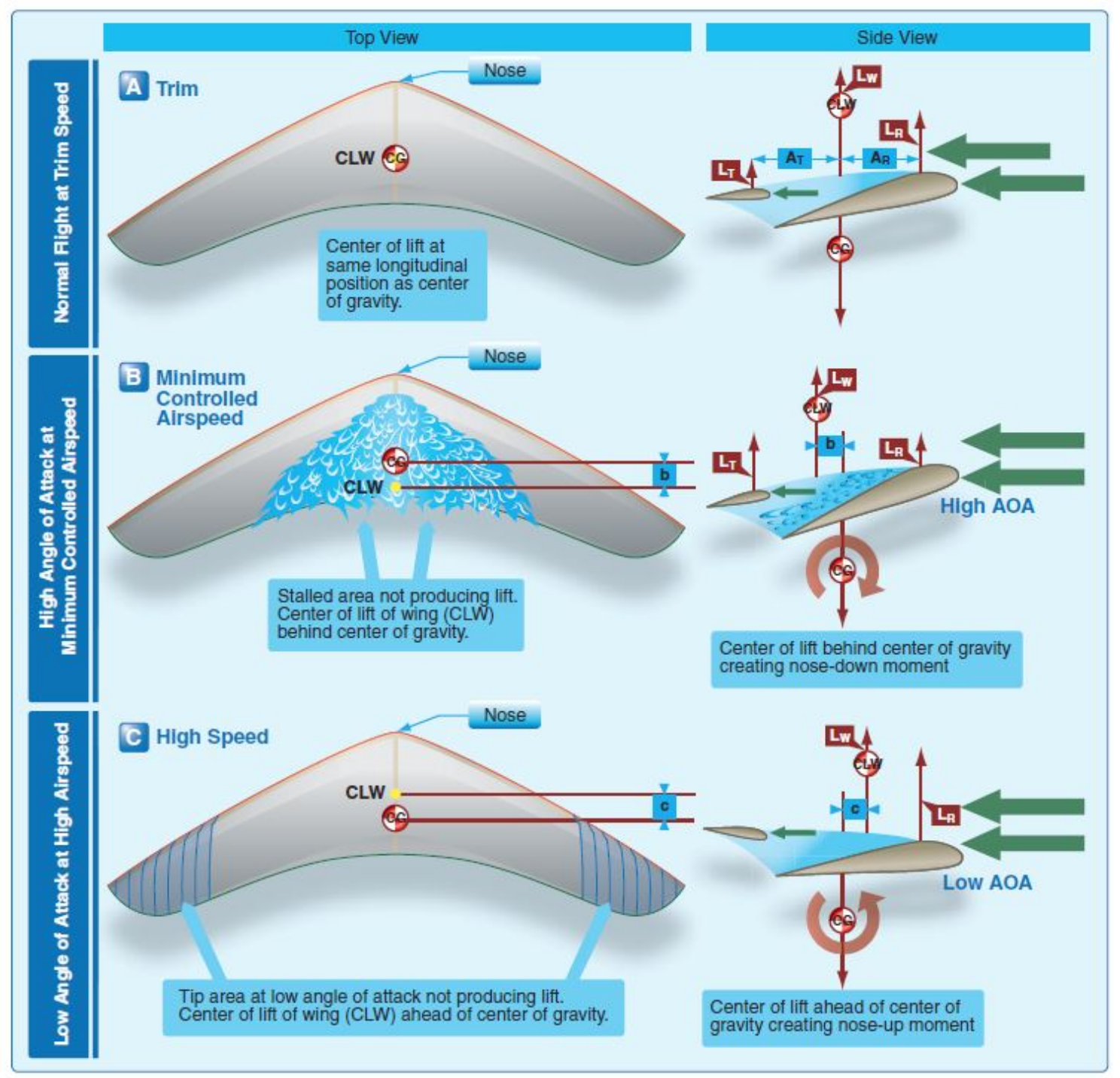

Figure 2.6: Lift generation at the wing root and wing tip for ultra-light wings [6].

Part $B$ of the figure above illustrates an ultra-light wing in a high angle of attack configuration with minimum controlled airspeed. Due to the angle of attack at the root being larger than the tip, stall will initially occur at the root. This leads to the lift being predominantly generated near the wing tip which in turn leads to a negative pitching moment reducing the angle of attack and allowing flow reattachment at the 
root. Figure 2.7 represents the lift coefficient as a function of the angle of attack for both the wing tip and wing root for the high angle of attack configuration [6]. Part $C$ of Figure 2.6 describes a high speed low angle of attack configuration. At low angles of attack, the wing tip lift contribution may be negligible or potentially negative. Therefore, the majority of the lift generated is upstream of the aerodynamic centre; this induces a positive pitching moment to increase the angle of attack.

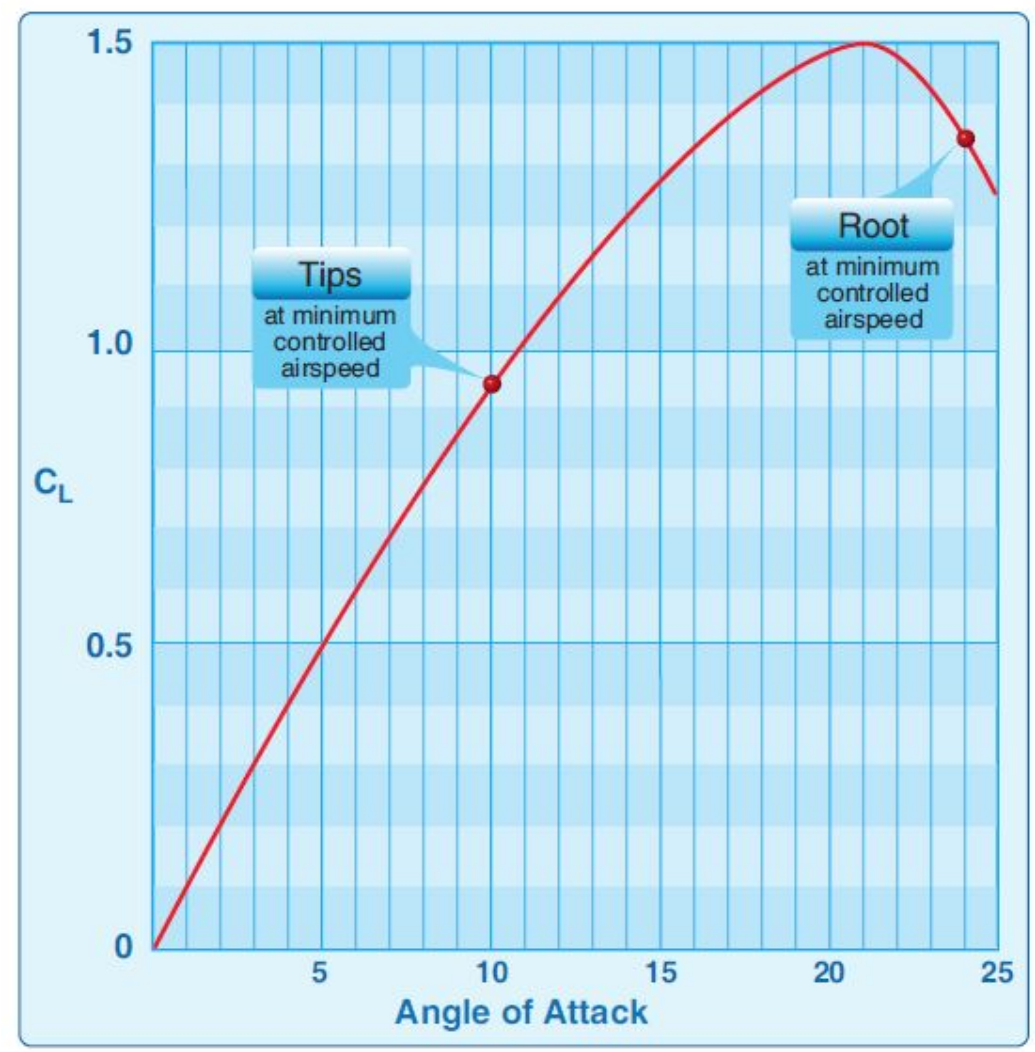

Figure 2.7: Lift curve at the minimum controlled airspeed for an ultra-light aircraft [6].

The aircraft's wing twist is crucial for maintaining longitudinal static stability. The magnitude of twist is dependent on the wing design; there are various ultra-light wings currently available and they are purpose-built for specific missions. 


\subsubsection{Modeling an Ultra-light Trike}

The existing conventions that are currently used to interpret aerodynamic loads on conventional fixed-wing aircraft are likewise used in ultra-light aircraft. Two sets of axes are defined on the aircraft: the body axes and the stability axes. The body axes are fixed to the aircraft's centre of gravity; they translate and rotate in conjunction with the aircraft. In the body frame of reference, the x-axis is conventionally parallel and coincident to the aircraft's longitudinal reference line [11 13$]$. The stability axes are also fixed to the aircraft's centre of gravity but they are prescribed with respect to the aircraft's reference condition [11-13]. In the case of steady-level flight with no bank and sideslip angles $(\phi=\beta=0)$, the alignment of the $\mathrm{x}$-axis with the freestream velocity is the reference condition. At this reference condition, the angle between the body and stability axes is the aircraft's angle of attack, $\alpha$. This is shown in detail in Figure 2.8 [13]. Once the aircraft is perturbed from this reference condition, the stability axes will no longer be aligned with the freestream velocity.

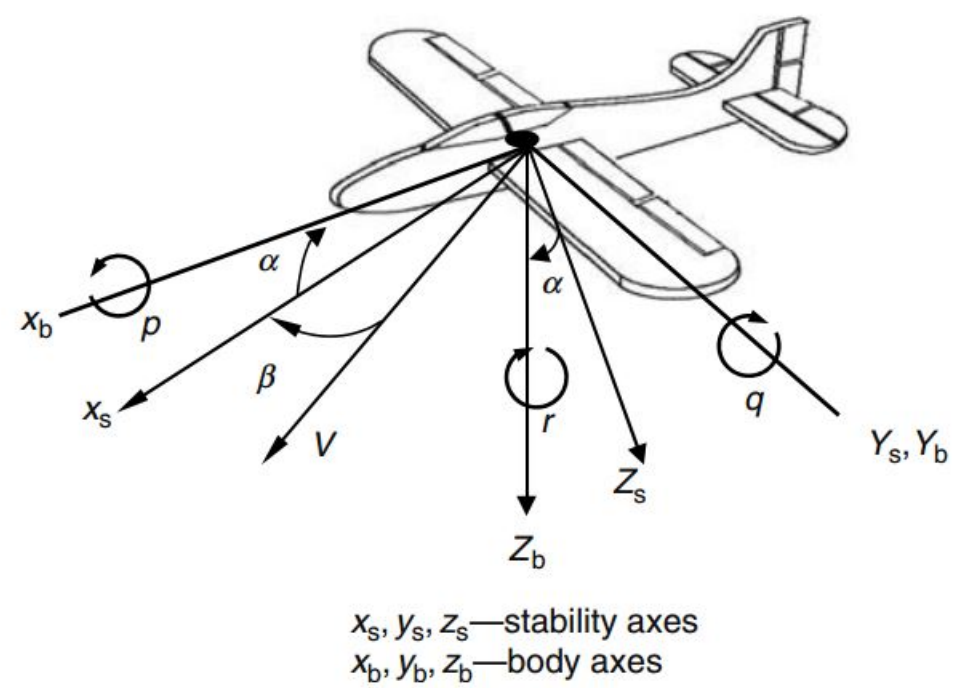

Figure 2.8: Conventions for aircraft body axes and stability axes 13. 
When analysing the forces and moments acting on the aircraft, either the body axes or stability axes may be used. In wind tunnel applications, it is dependent on the technique with which the balance measures the forces and moments. A balance may be set up to resolve the aerodynamic data in the body axes or in a tunnel-fixed axes [11]. If a tunnel-fixed axes system is used, the measured loads may be transformed to stability or body axes 14,15$]$.

The axial, lateral, and normal forces $(X, Y, Z)$ and the pitch, roll, and yaw moments $(M, \mathscr{L}, N)$ are defined with respect to the chosen set of axes. Nevertheless, the expressions for these forces and moments remains the same; they are generally expressed by nondimensional quantities shown in Table 2.1.

Table 2.1: Nondimensional force and moment coefficients for conventional aircraft.

\begin{tabular}{|c|c|}
\hline Forces & Moments \\
\hline$c_{X}=\frac{X}{\frac{1}{2} \rho V^{2} S}$ & $c_{M}=\frac{M}{\frac{1}{2} \rho V^{2} S \bar{c}}$ \\
$c_{Y}=\frac{Y}{\frac{1}{2} \rho V^{2} S}$ & $c_{\mathscr{L}}=\frac{\mathscr{L}}{\frac{1}{2} \rho V^{2} S b}$ \\
$c_{Z}=\frac{Z}{\frac{1}{2} \rho V^{2} S}$ & $c_{N}=\frac{N}{\frac{1}{2} \rho V^{2} S b}$ \\
\hline
\end{tabular}

Undertaking an analysis of aerodynamic loads on conventional fixed-wing aircraft is simplified by assuming that the aircraft is a rigid body. This assumption removes the need to consider the aircraft being subject to any aeroelastic effects. Further simplification is achieved by resolving the forces at the aircraft's centre of gravity.

The fundamentals of describing and quantifying loads on an ultra-light trike are similar to that of conventional aircraft. The existing methods of analysing aircraft stability are also directly applicable to ultra-light aircraft [8, 16]. However, 
some distinctions exist and must be recognized. Ultra-lights are primarily used for recreational purposes and thus the investigation of flight characteristics of these type of aircraft has been predominantly empirical [8]. Most existing theoretical research focuses on hang glider dynamics. Both hang gliders and ultra-light trikes are considered WSC aircraft. These type of aircraft do not have any active control surfaces such as ailerons, elevators, and rudders commonly found on conventional fixed-wing aircraft. Weight-shift control implies that the aircraft is controlled by a pilot's ability to change the aircraft's centre of gravity relative to the wing [6]. Therefore, the rigid body assumption described above does not apply to weight-shift control aircraft. Recent work in the modeling of hang glider dynamics has been undertaken by Cook and Spottiswoode [16 at Cranfield University and by Ochi 17 at the National Defense Academy of Japan.

In the Cook and Spottiswoode model, the centre of gravity of the aircraft is defined as a point in space that is located on a line connecting the centre of gravity of the pilot and the wing [16]. Body axes are defined at this centre of gravity location with the $\mathrm{x}$-axis parallel to the keel. A representation of this body axis system is illustrated in Figure 2.9. 


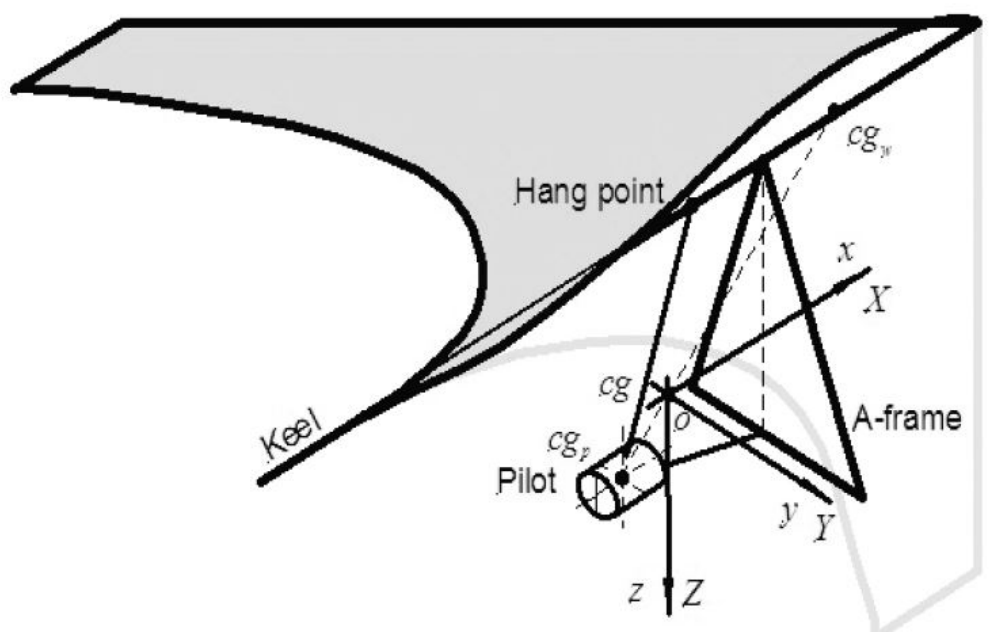

Figure 2.9: Cook and Spottiswoode hang glider model [16].

In order to accurately represent moments acting about the centre of gravity, it is essential to define body axes for the wing and pilot. These axes are shown in Figure 2.10. All forces and moments acting at the glider's centre of gravity are simply a summation of the forces and moments acting on both the wing and the pilot as shown in Table 2.2. The subscripts $w, p$, and $g$ refer to the load contribution of the wing, pilot, and gravity respectively.

Table 2.2: Hang glider forces and moments expressed about the centre of gravity.

\begin{tabular}{|c|c|}
\hline Forces & Moments \\
\hline$X=X_{w}+X_{p}+X_{g}$ & $M=\left(M_{w}-x_{w} Z_{w}+z_{w} X_{w}\right)+\left(M_{p}-x_{p} Z_{p}+z_{p} X_{p}\right)$ \\
$Y=Y_{w}+Y_{p}+Y_{g}$ & $\mathscr{L}=\left(\mathscr{L}_{w}+y_{w} Z_{w}-z_{w} Y_{w}\right)+\left(\mathscr{L}_{p}+y_{p} Z_{p}-z_{p} Y_{p}\right)$ \\
$Z=Z_{w}+Z_{p}+Z_{g}$ & $N=\left(N_{w}+x_{w} Y_{w}-y_{w} X_{w}\right)+\left(N_{p}+x_{p} Y_{p}-y_{p} X_{p}\right)$ \\
\hline
\end{tabular}




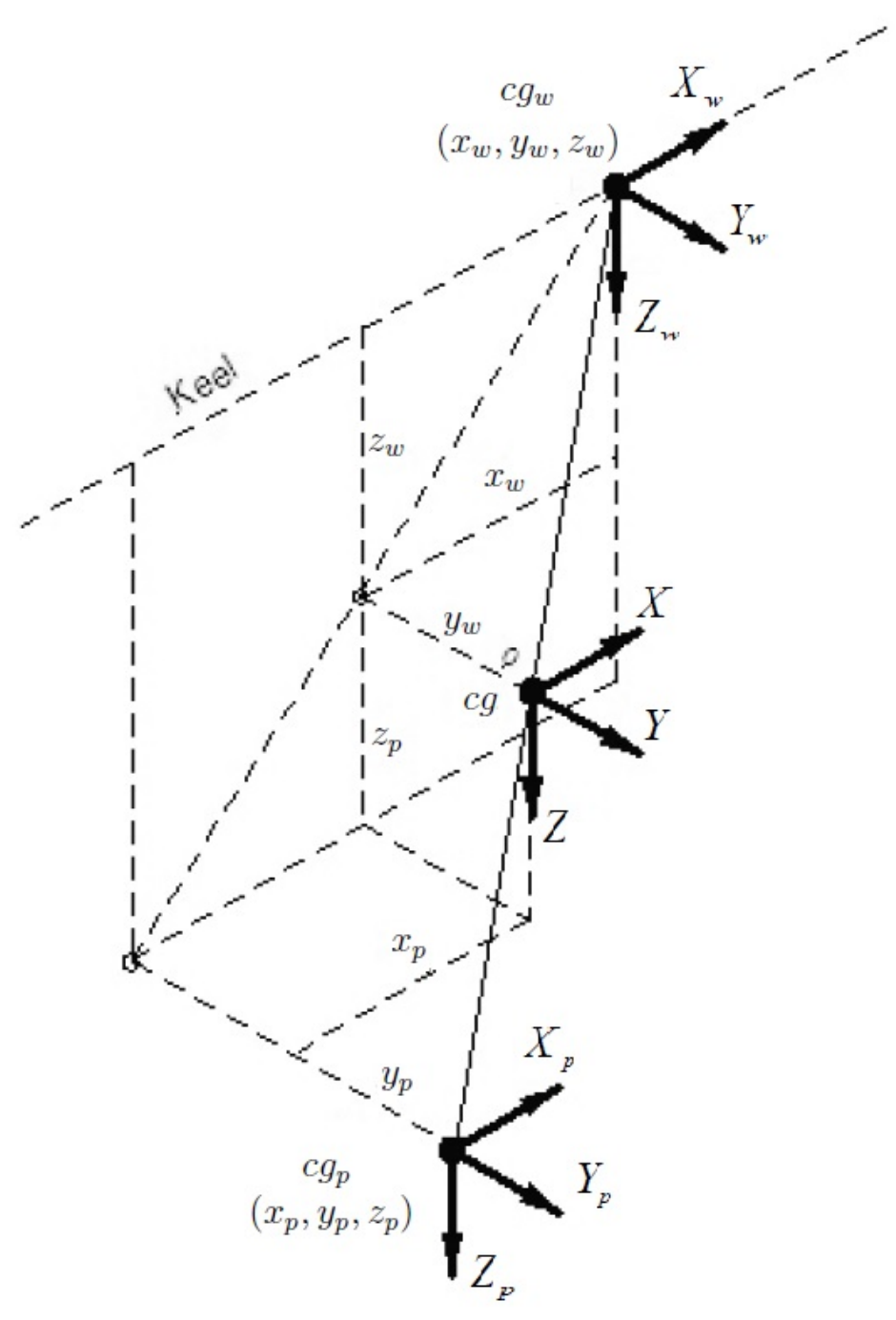

Figure 2.10: One-body simplification of the Cook and Spottiswoode hang glider model $[16$.

Cook and Spottiswoode simplify a two-body system (wing and pilot) to a one-body system in order to mathematically model hang glider dynamics. This creates some limitations on the model. First, the pilot is modelled as a cylinder having a uniform mass distribution. Second, the pilot's body axes are always parallel to the wing's body axes. Furthermore, the only control inputs that are defined are with respect to the pilot's immediate position. The pilot's longitudinal control input is defined as the angle between the A-frame and the yz-plane passing through the hang point. Their 
lateral control input is defined as the angle between the A-frame and the xz-plane. Finally, a key element that is ignored is the pilot's dynamics with respect to the wing [17]. In doing so, the glider can be modeled as a rigid body in its trim conditions.

Ochi models a hang glider as a two-body system [17]. Geometric and kinematic constraints are imposed and the interaction between the pilot and wing are considered [17]. Similar to the Cook and Spottiswoode one-body system, when modeling a twobody system, body axes are defined for both the pilot and wing. The key difference is that the axes of the pilot are not assumed to be parallel to the axes of the wing and thus can rotate independently of the wing. As a result, more realistic expressions of the forces acting on the system are achieved. This model is shown in Figure 2.11. The forces acting on the pilot in the longitudinal, lateral, and normal body directions are expressed as $X_{p}, Y_{p}$, and $Z_{p}$ respectively. Similarly, for the wing they are expressed as $X_{w}, Y_{w}$, and $Z_{w}$. For simplicity, it is convenient to relate the pilot's position, velocity, acceleration, as well as the external forces to the wing's frame of reference by the application of a rotation matrix [17]. A similar breakdown of the forces and moments shown in Table 2.2 were developed in the Ochi model with one important addition. Internal forces at the control bar, $F$, and at the hang point, $S$, were taken into account. The full development of the equations of the forces and moments are presented by Ochi 17]. 


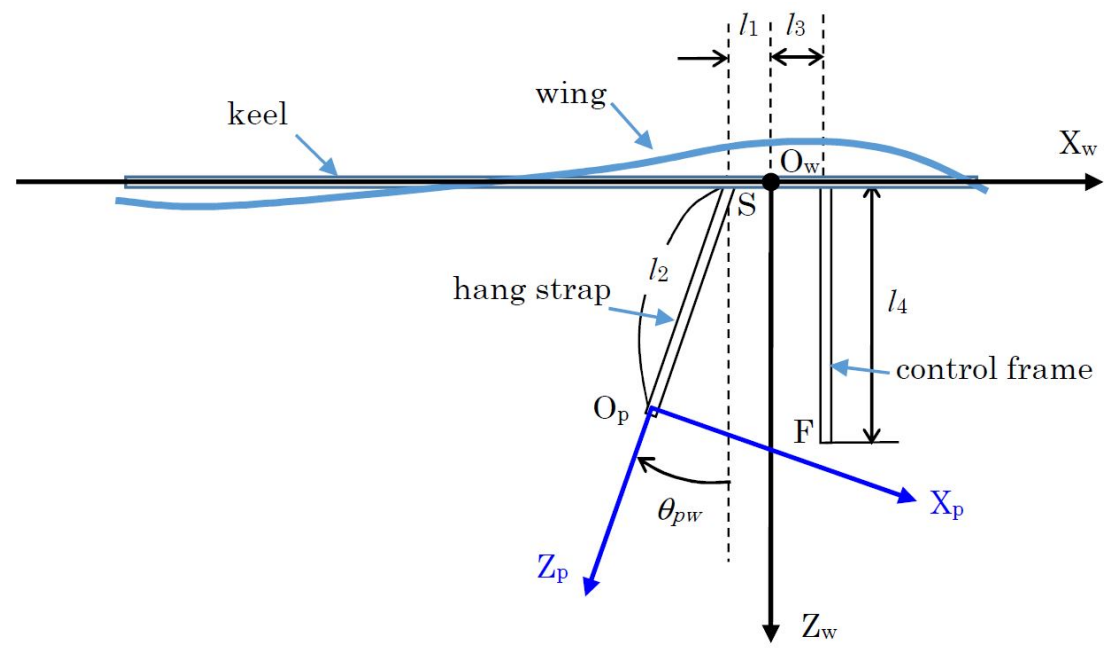

Figure 2.11: Ochi two-body hang glider model [17].

\section{$2.2 \quad$ Static Stability}

The static stability of an aircraft is best analyzed in two parts: longitudinal motion and lateral motion [11]. In terms of longitudinal motion, an aircraft is said to be in steady flight when the total pitching moment about its centre of gravity is zero. This is known as the trim condition. When any disturbance from a steady flight produces a restoring moment to bring it back to an equilibrium state, the aircraft meets the condition of longitudinal static stability [12]. Figure 2.12 illustrates the pitching moment about the centre of gravity of a conventional aircraft at various angles of attack. 


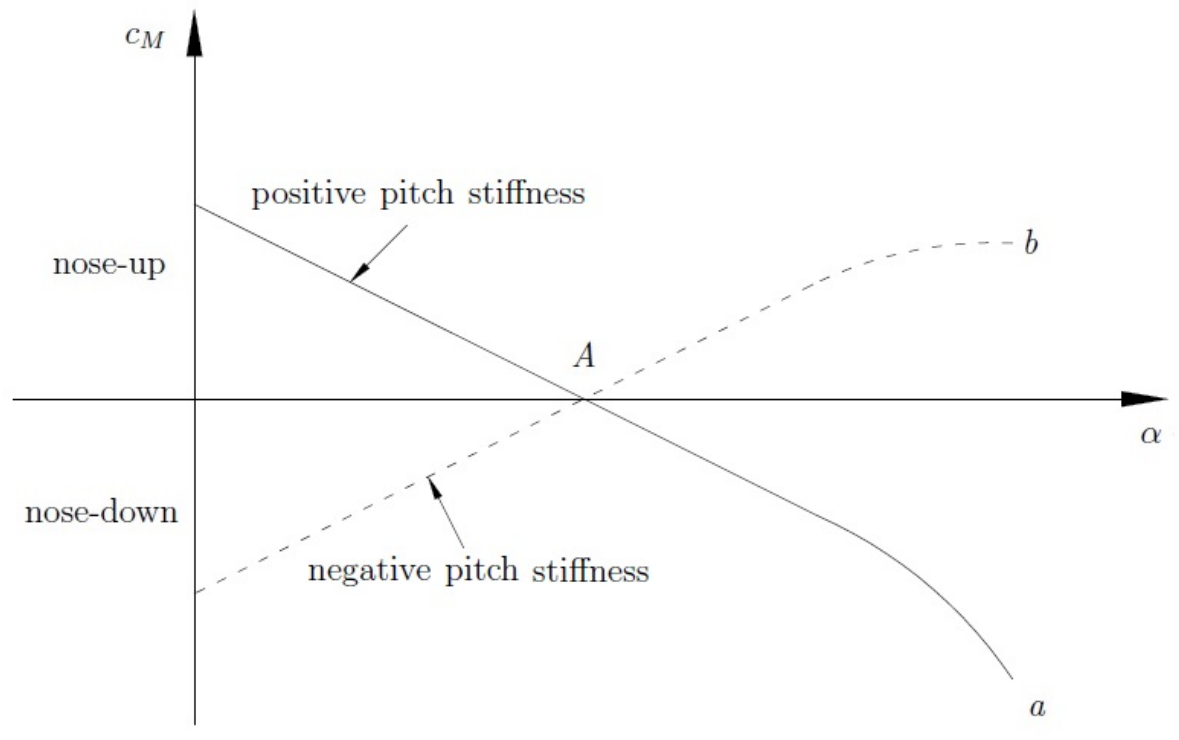

Figure 2.12: Pitch stiffness condition for longitudinal static stability (adapted from [11).

A positive pitching moment coefficient causes the aircraft to pitch nose-up whereas a negative $c_{M}$ will generate a nose-down pitching moment. In Figure 2.12 , point $A$ is the equilibrium or trim position. The negative slope in curve $a$ illustrates that at high angles of attack, the aircraft will have a nose-down pitching moment. At low angles of attack, $\alpha$, the tendency is to pitch nose-up. Therefore, the aircraft will continuously attempt to remain at its trim position $\left(c_{M}=0\right)[12$. This is referred to as a positive pitch stiffness [11]. The positive slope (or negative pitch stiffness) in curve $b$ indicates that the aircraft will continue to pitch nose-up at higher angles of attack and continue to pitch nose-down at lower angles of attack. Therefore, for the condition of longitudinal static stability to apply, it is required that $c_{M}=0$ at some angle of attack $\alpha$ and that $\partial c_{M} / \partial \alpha<0$ [11, 12. 
Similar to longitudinal static stability, any lateral motion of the aircraft should have counteracting moments that tend to return the aircraft to an equilibrium state in order for the condition of lateral static stability to hold true [12]. There are two conditions that must be satisfied:

$$
\begin{gathered}
\frac{\partial c_{N}}{\partial \beta}>0 \\
\frac{\partial c_{\mathscr{L}}}{\partial \beta}<0
\end{gathered}
$$

Equation 2.1 states that the change in yawing moment with respect to the sideslip (yaw) angle should be positive. In Figure 2.13, it is evident that a positive sideslip angle should induce a negative yaw moment in order to return the aircraft into the relative wind direction [12]. The second condition for lateral static stability is given by Equation 2.2. It illustrates the roll moment's dependency on the sideslip angle. When an aircraft is in a sideslip, an induced rolling moment is generated due to an asymmetric lift distribution. As seen in Figure 2.14, a negative $c_{\mathscr{L}_{\beta}}$ is required for a sideslip-induced roll moment to return the aircraft to an equilibrium state. The wing sweep, dihedral, twist, and its position relative to the aircraft's centre of gravity affect the lift distribution 11, 12. 


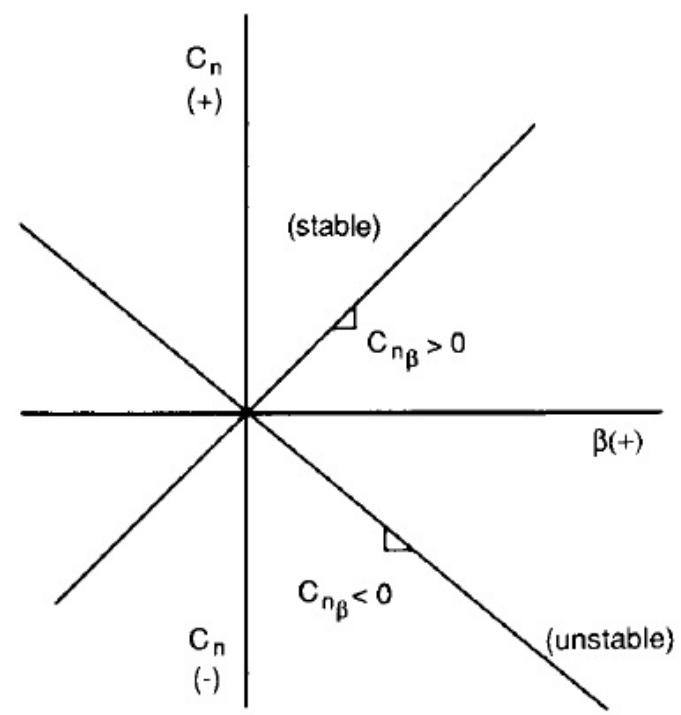

Figure 2.13: Requirement for yaw stability in a sideslip [12].

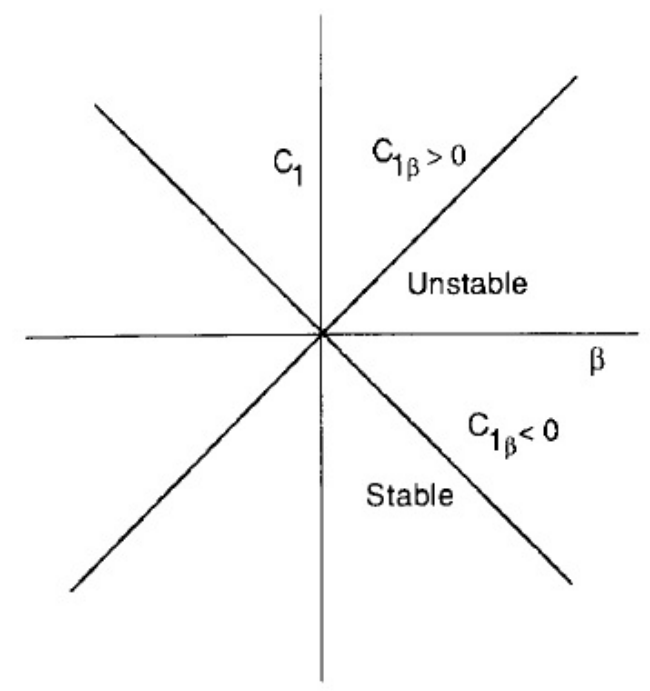

Figure 2.14: Requirement for roll stability in a sideslip [12].

When the aircraft is in a sideslip, the wing sweep causes one half of the aircraft's wing to be more exposed to the oncoming flow. This, in turn, will create more drag and lift on the more exposed wing. The increase in drag will generate a yaw moment that will attempt to yaw the aircraft into the direction of the oncoming flow as seen 
in Figure 2.15 [6]. Furthermore, from Figure 2.16, it can be seen that when the aircraft is in a banked state, a component of the aircraft's weight will act along the aircraft's lateral axis. A roll-induced sideslip will be created which will in turn change the direction of the oncoming velocity vector (i.e. it will no longer be acting along the longitudinal axis of the aircraft). Thus, an asymmetric lift distribution will be generated. In order for Equation 2.2 to be satisfied, $L_{1}$ must be greater than $L_{2}$.

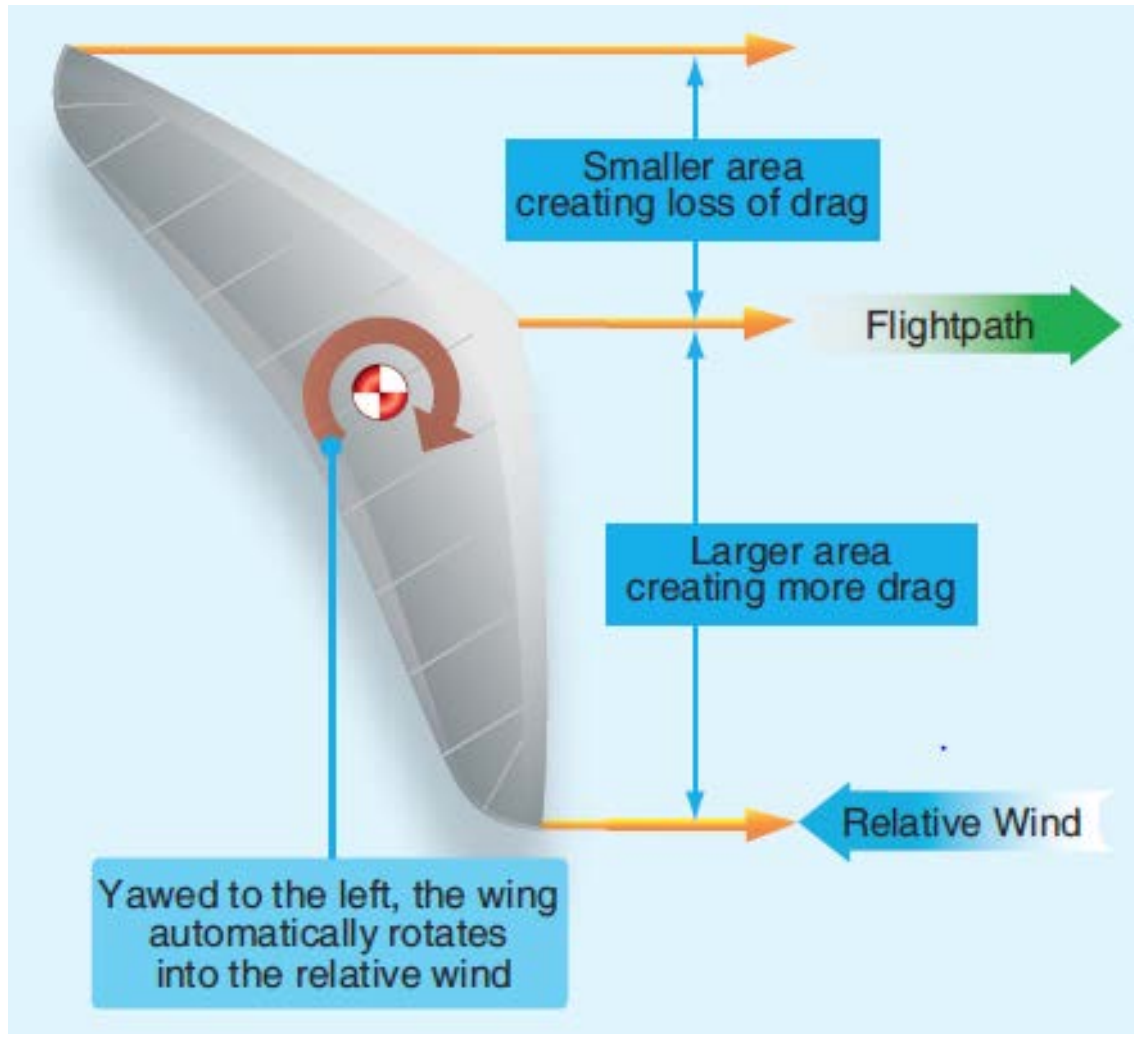

Figure 2.15: Restoring yaw moment generated by wing sweep when the aircraft is in a sideslip [6]. 


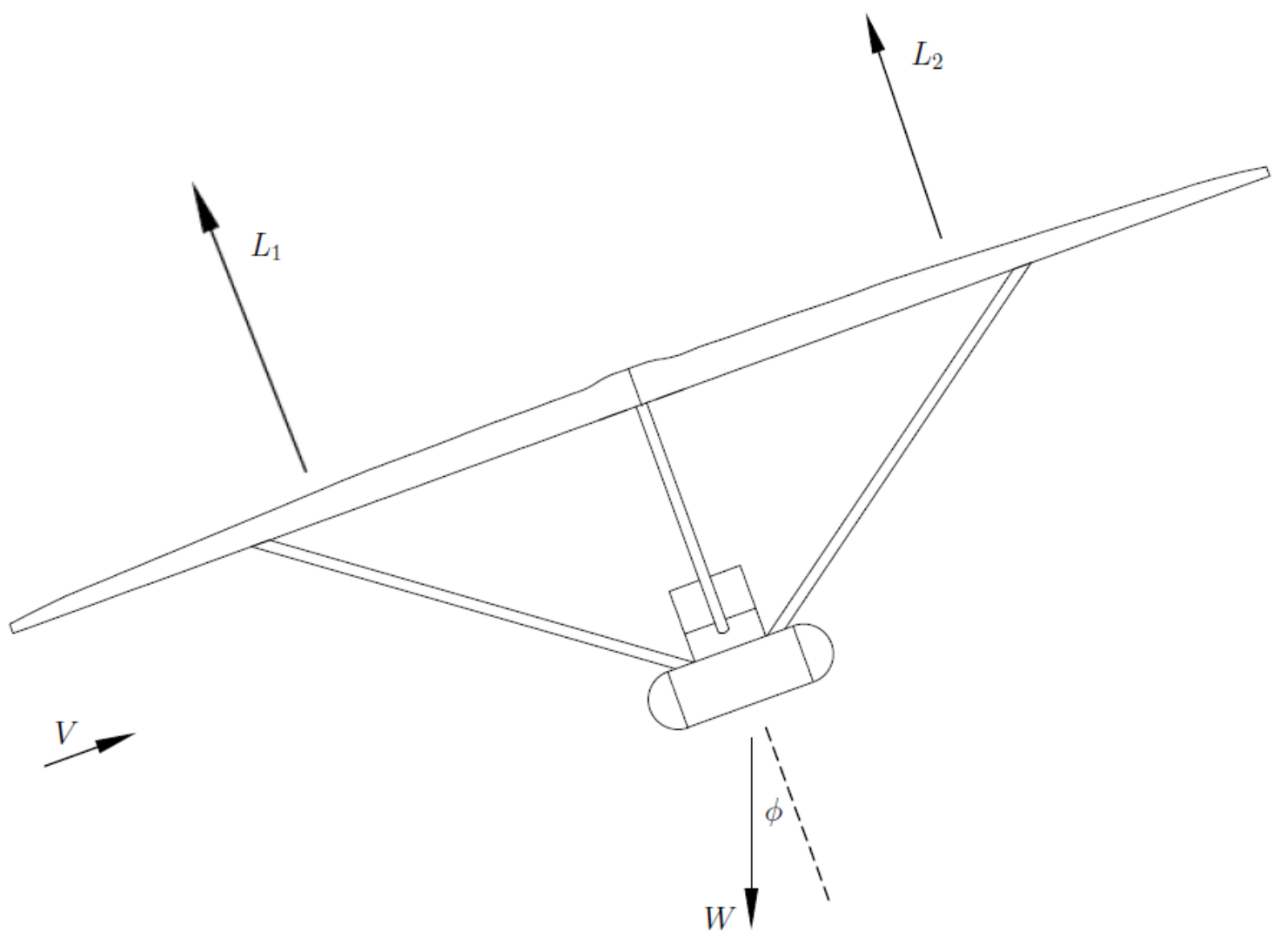

Figure 2.16: Restoring roll moment when the aircraft is in a sideslip.

When the aircraft is in a purely banked state $(\phi \neq 0, \beta=0)$, it is not considered to be a static stability problem, i.e.

$$
\frac{\partial c_{\mathscr{L}}}{\partial \phi} \equiv c_{\mathscr{L}_{\phi}}=0
$$

A change in the bank angle does not change the aerodynamic forces and moments acting on the aircraft so long as the aircraft's longitudinal axis is parallel to the oncoming flow [11]. It should be noted that the rolling moment does change with respect to the roll rate and therefore this is a dynamic problem. 


\subsubsection{Longitudinal Static Stability Derivatives}

The conservation of momentum equations of an aircraft are linearized by the application of the small-disturbance theory [12]. This theory assumes that the aircraft's reference condition is known and only small motions about this reference point occur. When low magnitude disturbances occur, the aerodynamic loads can be represented by linear functions of these disturbances. Steady-level flight is commonly used as the reference state for the application of the small-disturbance theory [11. When applying this theory, the linearized equations can be differentiated with respect to various aerodynamic variables such as angle of attack or change in forward speed. The complete development of the small-disturbance equations of motion can be found in various well known aircraft dynamics textbooks [11,12, 18.

In the previous section, the forces $X$ and $Z$ were defined in the longitudinal and normal directions respectively using a body frame of reference. The moment $M$ acts about the lateral axis of the aircraft. The change in the non-dimensional coefficients $c_{X}, c_{Z}$, and $c_{M}$ with respect to the angle of attack yield the longitudinal static stability derivatives:

$$
\begin{gathered}
c_{X_{\alpha}}=\frac{\partial c_{X}}{\partial \alpha} \\
c_{Z_{\alpha}}=\frac{\partial c_{Z}}{\partial \alpha} \\
c_{M_{\alpha}}=\frac{\partial c_{M}}{\partial \alpha}
\end{gathered}
$$




\subsubsection{Lateral Static Stability Derivatives}

Similar to the longitudinal case, the lateral force coefficient $c_{Y}$, the yaw moment coefficient $c_{N}$, and the roll moment coefficient $c_{\mathscr{L}}$ are also differentiated with respect to an aerodynamic variable to yield stability derivatives. As explained earlier, a change in the bank angle does not change the aerodynamic forces or moments, therefore the lateral static stability is only dependent on the sideslip angle, $\beta$. The lateral force and moment derivatives are given by:

$$
\begin{gathered}
c_{Y_{\beta}}=\frac{\partial c_{Y}}{\partial \beta} \\
c_{N_{\beta}}=\frac{\partial c_{N}}{\partial \beta} \\
c_{\mathscr{L}_{\beta}}=\frac{\partial c_{\mathscr{L}}}{\partial \beta}
\end{gathered}
$$

\subsection{Similarity Parameters}

In the field of experimental fluid mechanics, conducting experiments on a large prototype is often costly and impractical [19]. There are costs associated with the development of the prototype as well as the instrumentation required to measure the desired data. If numerous design information is required, the cost and availability of specific measurement devices may hinder the experiments. Furthermore, the placement of all instrumentation on a prototype must be executed in a careful manner as to not act as an impediment to the flow. It may also prove difficult, if not impossible, to locate a test facility that can accommodate a large prototype. 
An alternative that is commonly used to overcome the aforementioned obstacles is the application of dimensional analysis to scale the model to fit into smaller test facilities 1921$]$. A design can be scaled-down in order to run experiments in a safe and cost effective manner in smaller wind tunnels or water channels. Experiments that are run with scale models can effectively provide information about the behaviour of the full-scale model if scaling laws are correctly implemented 19 21]. This concept is known as similarity. In order for a scale model to be representative of its fullscale counterpart with regards to performance and stability, it must be geometrically, kinematically, and dynamically similar 21,22].

\subsubsection{Geometric Similarity}

In order to satisfy the condition of geometric similarity, the physical dimensionless parameters of the scale-downed model must match those of the full-scale model. In terms of aircraft, these parameters will include the wing's aspect ratio (AR), sweep angle, dihedral (or anhedral) angle, along with the thickness-to-chord ratio of the airfoil. When a model is scaled down, the geometric similarities are preserved [22].

\subsubsection{Kinematic Similarity}

Kinematic similarity is achieved when the velocity ratio, $V / V_{\infty}$, is identical for both bodies when they are compared in a normalized coordinate system. Furthermore, the direction of the velocity vector at geometrically similar locations is the same (i.e. streamlines do not change with the scale); the ratio of their magnitudes is constant 19,22 . 


\subsubsection{Dynamic Similarity}

Dynamic similarity is the third condition that must be satisfied for complete similarity and it is defined by the properties of the fluid flow. When comparing the flow field over a full-scale aircraft to that of a scaled-down model, they are only considered to be dynamically similar if the following conditions are met $10,19,22$ :

- The direction of the force vector at geometrically similar locations is the same; the ratio of their magnitudes is constant.

- The nondimensional pressure distributions, $P / P_{\infty}$, is identical for both bodies when they are compared in a normalized coordinate system.

- The aerodynamic force and moment coefficients listed in Table 2.1 remain unchanged.

The conditions above can be confirmed by ensuring that the model and flow field conditions are geometrically similar and by matching the relevant dimensionless flow parameters [10]. An extensive amount of these parameters have been developed in the context of fluid mechanics. The Reynolds number (Eq.2.10) and Mach number (Eq. 2.11) are but a few of these dimensionless coefficients; they are also the most dominant similarity parameters [10].

$$
\begin{gathered}
R e=\frac{\rho V L}{\mu} \\
M_{\infty}=\frac{V}{c}
\end{gathered}
$$

When the flow field is incompressible $\left(M_{\infty}<0.3\right)$, the necessity of matching the Mach number is no longer required. The Reynolds number becomes the key parameter to match. 20, 22] The nondimensional force and moment coefficients defined in Table 2.1 are functions of the Reynolds number: 


$$
C_{F}=f(R e)
$$

From Equation 2.12, in can be concluded that [19]:

$$
\text { if } R e_{m}=R e_{p} \text { then } C_{F_{m}}=C_{F_{p}}
$$

where the subscript $F, m$, and $p$ represent an arbitrary force or moment under investigation, the model, and the prototype respectively.

\subsection{Parameter Estimation}

As wind-tunnel experiments are conducted under static conditions, the aerodynamic loads only become a function of the immediate flight condition and aircraft configuration 23]. The flight condition refers to the aircraft's orientation with respect to the oncoming flow (i.e. angle of attack, $\alpha$, and sideslip angle, $\beta$ ). The aircraft configuration is the consideration of physical characteristics of the aircraft such as landing gear and wing geometry; it also considers control surface deflections [23]. Any dynamic motion such as angular rates and accelerations are not considered in static tests. A least squares linear regression algorithm is used to determine aerodynamic derivatives in static testing expressed as 23, 24]:

$$
y=a x+b
$$

where

$$
\begin{gathered}
a=\frac{\sum_{i=1}^{n}\left(x_{i}-\bar{x}\right)\left(y_{i}-\bar{y}\right)}{\sum_{i=1}^{n}\left(x_{i}-\bar{x}\right)^{2}} \\
b=\bar{y}-a \bar{x}
\end{gathered}
$$


The sample mean for the input and output are expressed as $\bar{x}$ and $\bar{y}$ respectively. The steady-state nature of wind-tunnel experiments for static stability derivative analysis allows for this least squares regression method to be applied [23]. All results obtained are with respect to a single independent variable $(\alpha$ or $\beta)$. 


\section{Chapter 3}

\section{Experimental Setup and Procedures}

\subsection{Overview}

In this chapter, the development of the ultra-light aircraft models that are to be tested in a wind-tunnel is described. An ultra-light wing model was manufactured and is to be tested in isolation for the purpose of validating a pre-existing CFD simulation of the wing in question. An ultra-light wing and fuselage were also fabricated for the purpose of aerodynamically characterising the aircraft and component interactions.

The wind-tunnel facility along with the associated instrumentation that was used to run all experiments are also described. The experimental methodology for all test cases is explained and the data reduction is developed in this chapter.

\subsection{Wind-Tunnel Configuration}

The experiments were performed in the low-speed wind-tunnel at Carleton University (Room ME 3224). It is a closed-circuit wind-tunnel with a contraction ratio of 9:1. A fan located opposite the test section draws in air within the tunnel. The air 
expands through a diffuser and settling chamber. The flow then accelerates through the contraction and into the test section where the model is mounted. A diagram of the wind-tunnel can be seen in Figure 3.1. The fan rotates at a maximum rate of $60 \mathrm{~Hz}$; this corresponds to a maximum freestream velocity of approximately 60 $\mathrm{m} / \mathrm{s}$ in the test section. The fan frequency is managed by a control panel directly across from the test section. A test section was purpose-built for this tunnel. The specifications of the test section are listed in Table 3.1 .

Table 3.1: Test section specifications

\begin{tabular}{|c|c|}
\hline Length & $183 \mathrm{~cm}[72 \mathrm{in}]$ \\
\hline Width & $76 \mathrm{~cm}[30 \mathrm{in}]$ \\
\hline Height & $51 \mathrm{~cm}[20 \mathrm{in}]$ \\
\hline Cross-sectional Area & $3871 \mathrm{~cm}^{2}\left[600 \mathrm{in}^{2}\right]$ \\
\hline
\end{tabular}

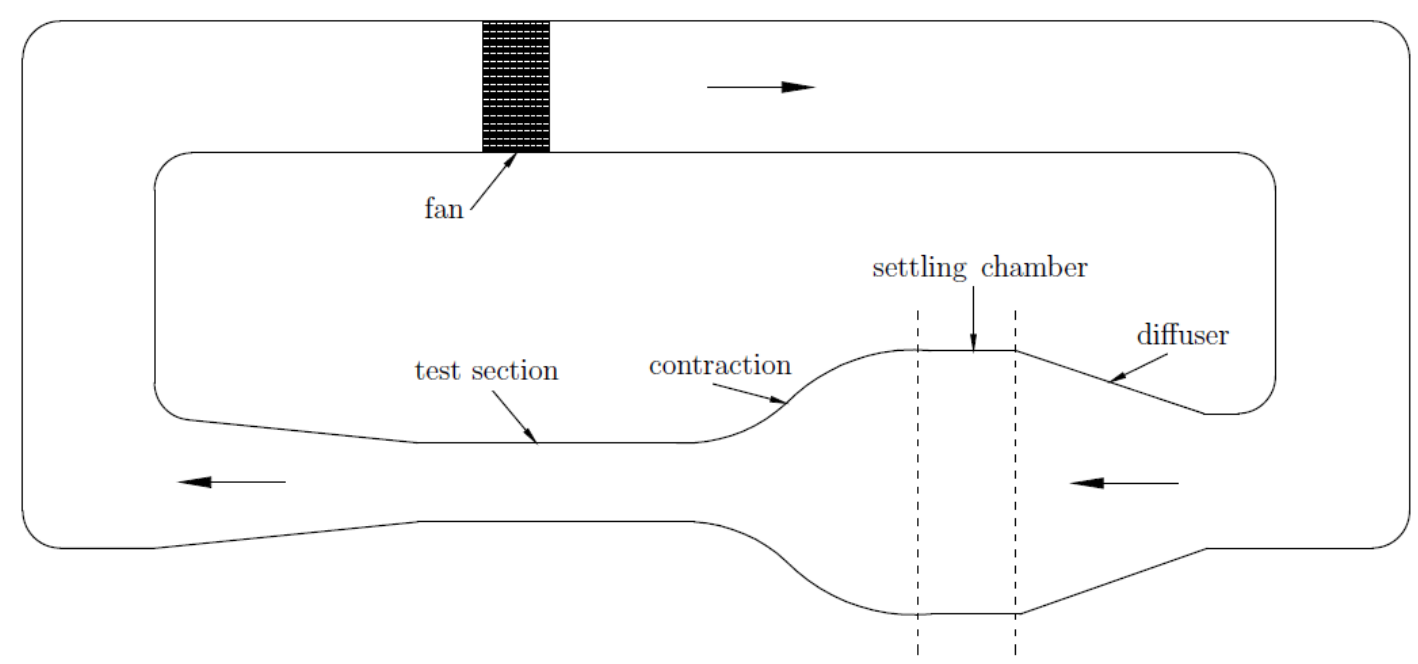

Figure 3.1: Schematic of the Carleton University closed-circuit wind-tunnel (not to scale). 


\subsection{Model Design and Manufacture}

\subsubsection{Ultra-light Wing}

The ultra-light wing model that was used to run wind tunnel experiments is $\frac{1}{28}$ scale model of the PROFI TL 14 double surface flexible wing for a two-seater trike aircraft. This full scale wing is manufactured by Ukrainian-based ultra-light aircraft company AEROS Ltd. The relevant information about the PROFI TL 14 wing can be found in the Table 3.2 [25].

Table 3.2: Key data for the full scale PROFI TL 14 ultra-light wing.

\begin{tabular}{|c|c|}
\hline Sail area $\left(\mathrm{m}^{2}\right)$ & 14.5 \\
\hline Wing span $(\mathrm{m})$ & 10.0 \\
\hline Aspect ratio & 6.9 \\
\hline Max airspeed $(\mathrm{km} / \mathrm{h})$ & 140 \\
\hline Stall speed with max load (km/h) & 52 \\
\hline Range of operating overloads (G-force) & $+4 /-2$ \\
\hline Ultimate tested strength (G-force) & $+6 /-3$ \\
\hline Total load max (kg) & 450 \\
\hline Mass of wing (kg) & 60.0 \\
\hline
\end{tabular}

The scaled-down wing was 3D printed from ABSplus-P430 thermoplastic using a Dimension 1200es 3D printer [26]. Volume restrictions of the printer lead to the starboard and port halves of the wing to be printed separately. A computer-aided 
design (CAD) model of the full scale wing was developed in SolidWorks from detailed measurements of all components of its internal structure (ribs, spars, keel, etc.). The CAD model was scaled down and modifications were made at the root of the wing to facilitate the joining of both halves. Two $\frac{1}{8}$ inch holes were made along the span of the wing at the root to accommodate dowel pins as illustrated in Figure 3.2 . Three other holes were included on the lower surface of the wing for the purpose of attaching the wing to the fuselage. Adhesive was used to bond the dowel pins to both sections of the wing.

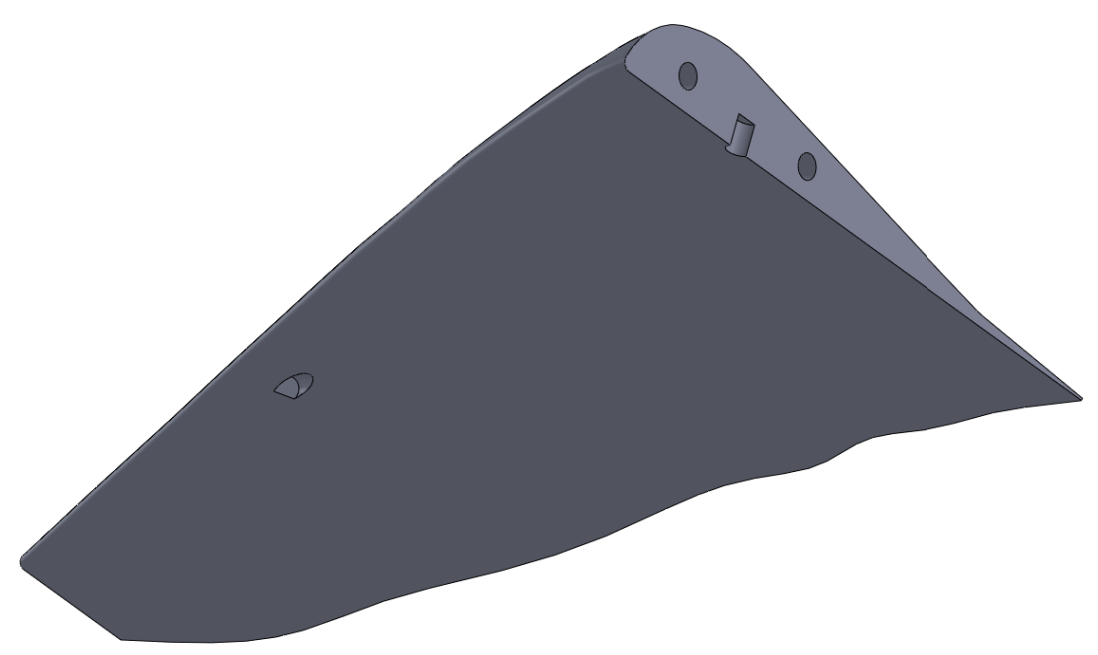

Figure 3.2: Modification to the scaled-down ultra-light wing.

Two sets of wings were printed and tested. The first wing, seen in Figure 3.3 is the original CAD model developed. This wing is used to perform wind-tunnel experiments on the aircraft as a whole. Modifications to the trailing edge of the wing at its root were made and a second wing was printed. The purpose of the change in wing geometry shown in Figure 3.4 is to facilitate the generation of a mesh for CFD simulations. The rounded edges lead to difficulty in generating a valid mesh therefore these changes were required [27]. 


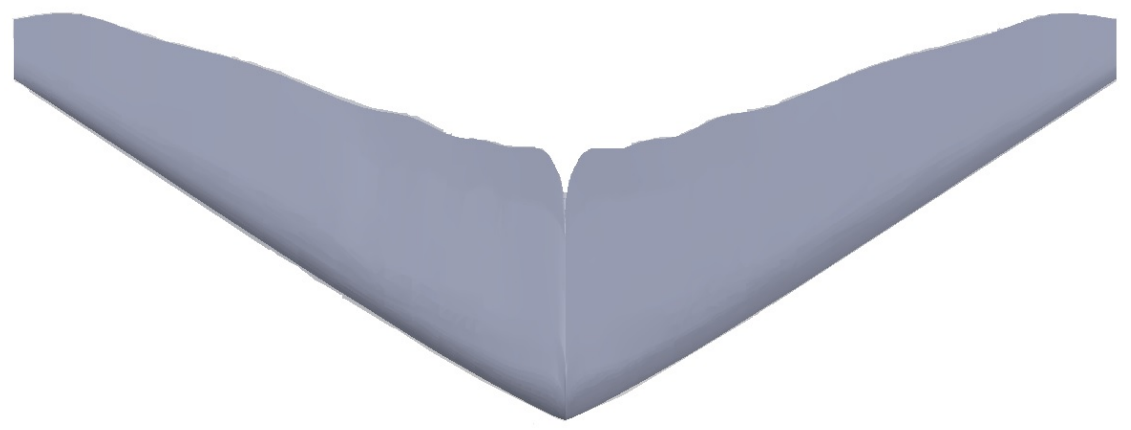

Figure 3.3: CAD model of the PROFI TL 14 ultra-light wing.

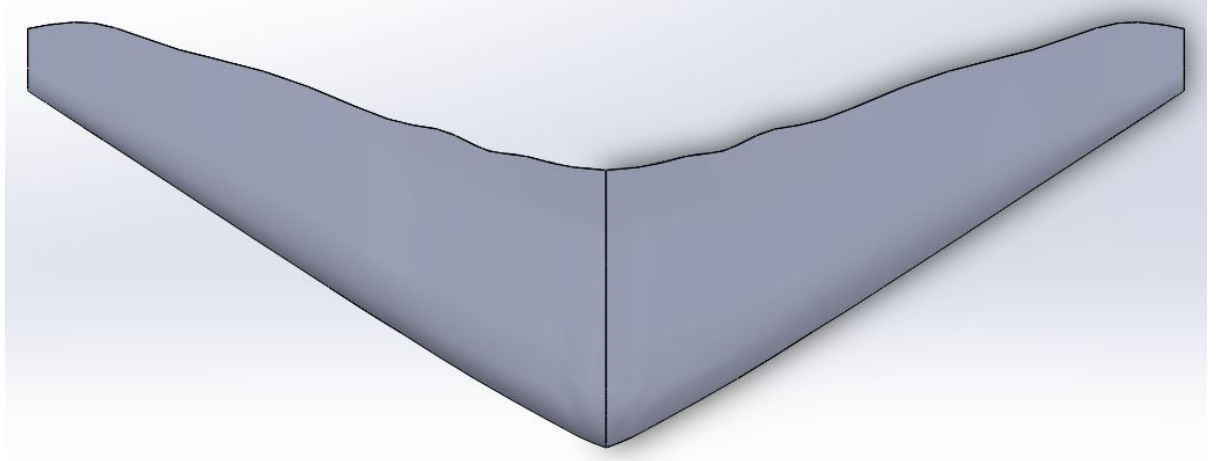

Figure 3.4: CAD model of the PROFI TL 14 ultra-light wing with the trailing edge at the root modified.

The experiments were undertaken with the modified wing in isolation rather than with the fuselage attached. The purpose of this experiment was to validate the CFD model by comparing experimental results with computational results.

\subsubsection{Ultra-light Wing and Fuselage}

The fuselage that was to be tested in the wind-tunnel is a preliminary design developed by the industry partners. The CAD model of the fuselage is shown in the Figure 3.5 . The fuselage was also 3D printed out of acrylonitrile butadiene styrene (ABS) plastic. 


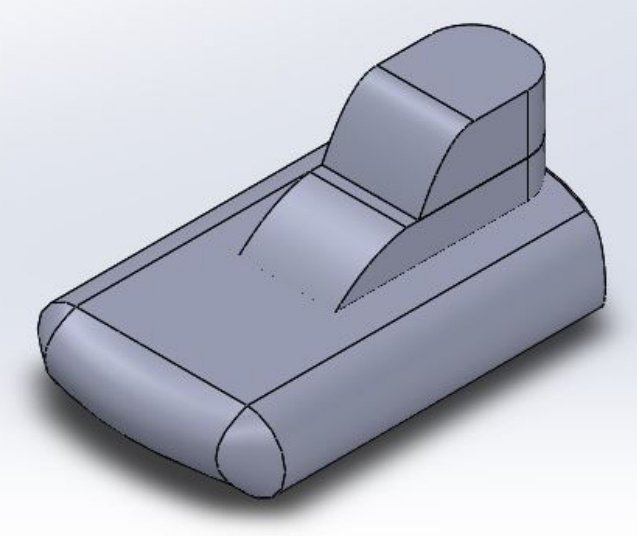

Figure 3.5: Preliminary design of the fuselage for the ultra-light aircraft.

Three $1 / 8$ inch diameter steel rods were used to connect the fuselage and wing as shown in Figure 3.6. The centre rod connected the fuselage's centre of gravity to the wing at its root's quarter-chord location. The spanwise location of the two outer rods are at a distance of approximately two-thirds of its half-span from the root. Their chord-wise location are at approximately $10 \%$ from the leading edge. The location of all rods was chosen to mimic the PROFI TL 14 wing as accurately as possible. These rods were not geometrically similar to the full size aircraft; the rods used to connect the fuselage to the wing are airfoil shaped as opposed to the circular rods that were used on the scale model. The airfoil shaped rods could potentially have an impact on both performance and lateral stability and thus further analysis would be required. The aircraft model is illustrated in Figure 3.6. From this figure, it can be observed that the wing and fuselage are rigidly connecting to each other. Therefore, the body axes of the fuselage and the wing will remain parallel; this is analogous to the Cook and Spottiswoode one-body system illustrated in Figures 2.9 and 2.10 16]. Furthermore, the entire structure is assumed to be rigid. 

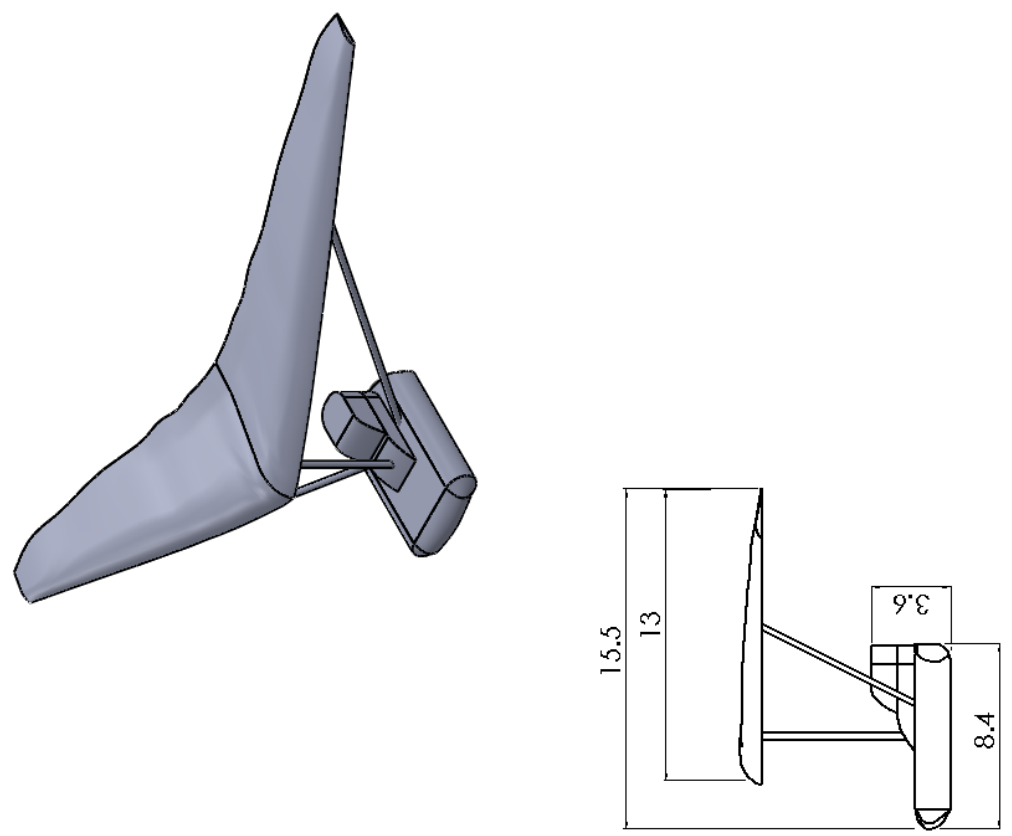

(2)
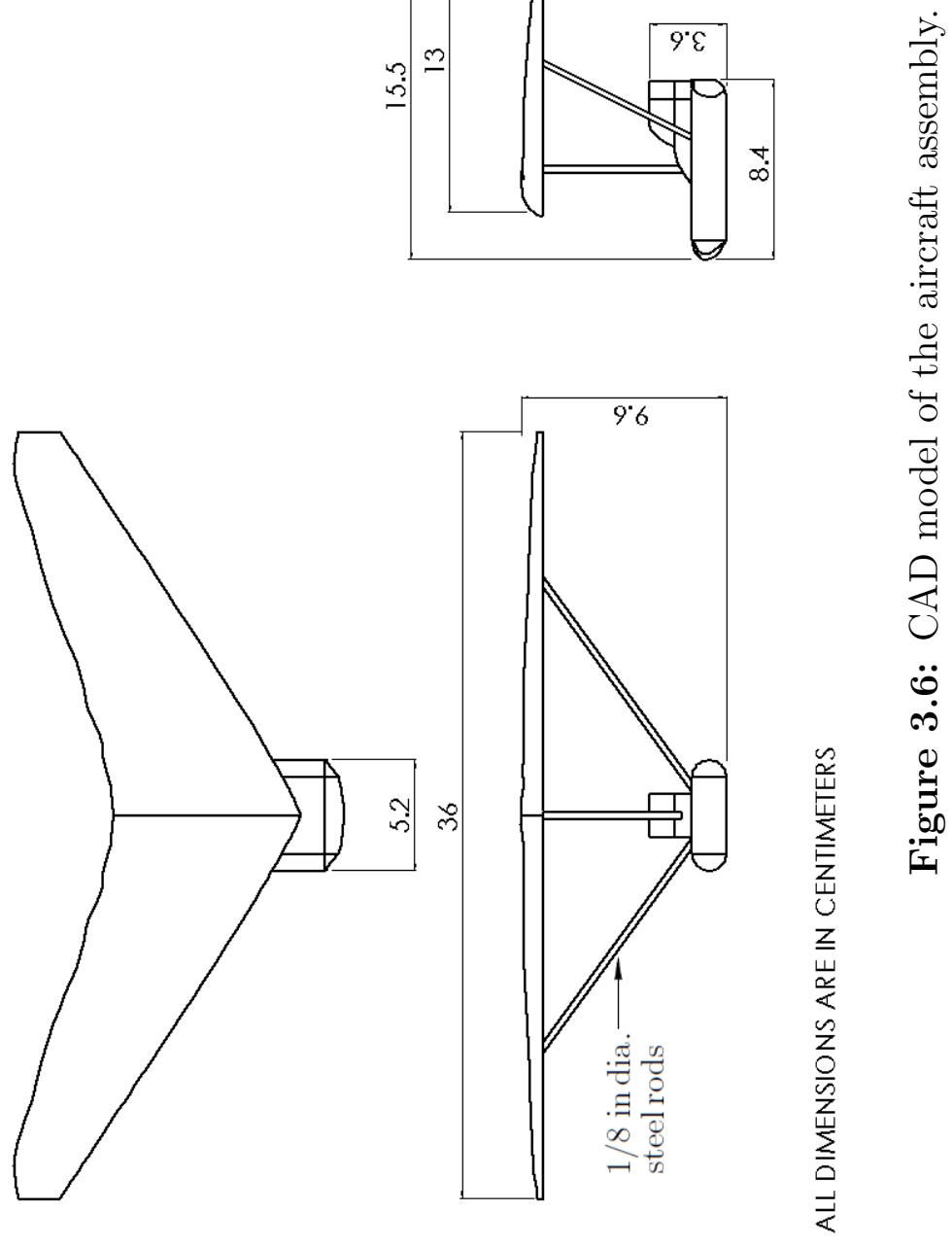


\subsubsection{Blockage Effects}

The 3D printed model possesses a wing span of approximately $36 \mathrm{~cm}$. The frontal area of the aircraft model is approximately $53 \mathrm{~cm}^{2}$. This equates to approximately $1.4 \%$ solid blockage in the cross-sectional area of the test section. It is important that the solid blockage area in the test section remain below $5 \%$ 20, 22, If the blockage area is too large, the airspeed around the model must increase significantly in order for mass to be conserved (continuity) [22]. Simple corrections may be made for low blockage areas $(<5 \%)$ by including the velocity increase to the freestream velocity $(V+\Delta V)$. This type of correction may not be suitable for larger blockage areas and a more appropriate for blockage correction method will be required. Various empirical solid blockage correction methods exist but are specific to the test section and model geometry [20,28]. In order to avoid the development of a suitable correction method, the frontal area of the model was kept relatively low 20,22 .

Another form of blockage that was considered when sizing the model is wake blockage. The velocity of the wake generated behind the model is less than the freestream velocity. Once again, in order to maintain continuity, the speed of the flow behind the model and that outside of the wake must increase. In Figure 3.7, the dashed line is a hypothetical representation of the fully developed uniform flow if no model was present. The solid line is a more representative illustration of the flow downstream of the model. The velocity in the wake is less compared to the freestream whereas the velocity outside of the wake is greater [20]. 


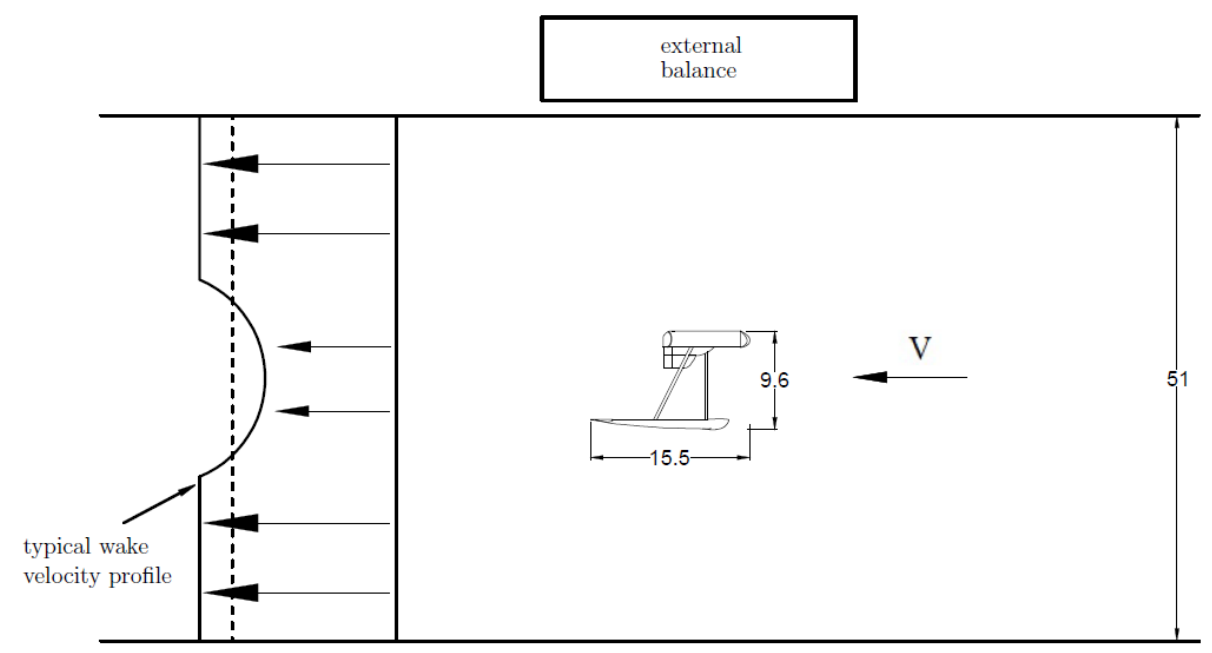

ALL DIMENSIONS ARE IN CENTIMETERS

Figure 3.7: Representation of the wake blockage effects within the test section.

This increase in velocity translates to a decrease in pressure downstream. Thus, a pressure gradient exists between the model and its wake which in turn causes the measured drag force to be greater. The wake blockage can be neglected in an open test section whereas it must be considered in a closed test section due to the flow not having the ability to expand in order to adjust for the pressure changes [20]. In closed test sections, similar to blockage effects, various methods of quantifying wake blockage exist $[20,29,30$. Maskell developed a wake blockage correction by considering momentum effects outside of the wake illustrated in Figure 3.7 [30]. An expression to quantify the wake blockage, $\varepsilon_{w b}$, was developed [30]:

$$
\varepsilon_{w b}=\frac{S}{4 A} c_{D_{u}}
$$

where $S, A$, and $c_{D_{u}}$ are the wing reference area, the cross-sectional area of the test section, and uncorrected drag coefficient respectively. The total blockage, $\varepsilon_{t}$, can be expressed as the summation of the wake blockage and solid blockage [20,29]: 


$$
\varepsilon_{t}=\varepsilon_{w b}+\varepsilon_{s b}
$$

For unconventional geometries in a wind tunnel, an initial estimate for the total blockage may be expressed as [20]:

$$
\varepsilon_{t}=\frac{1}{4} \frac{\text { frontal area of model }}{A}
$$

Once the total blockage effect is known, the corrected velocity, $V_{c}$, is given by [20]:

$$
V_{c}=V\left(1+\varepsilon_{t}\right)
$$

\subsubsection{Trip Strip}

The 3D printed parts have surface roughnesses inherent to the printing process. If these models were scaled up to their actual size, the roughness would scale up accordingly and geometric similarity would not be maintained. Therefore, all 3D printed parts were sanded to decrease roughness. Moreover, full scale ultra-light aircraft will experience Reynolds numbers in the order of $10^{6}$ (See Appendix C). At these high Reynolds numbers, transition in the boundary layer will occur rapidly [31. The Reynolds numbers that are obtained in the wind tunnel are too low to suitably maintain dynamic similarity. Thus, the addition of a boundary layer trip near the leading edge of the model's wing and fuselage is essential in order to overcome this obstacle; this is a widely used method in wind-tunnel tests $20,32,34$. Trip strips can be any surface roughness that is added to a specific chord-wise location on the wing in order to trip the boundary layer. In other words, it transitions the boundary layer from laminar to turbulent. Although a laminar boundary layer causes less friction

drag, the energy in a laminar boundary layer is significantly less than the energy within a turbulent boundary layer. Therefore, if a laminar boundary layer encounters 
an adverse pressure gradient, it may separate more readily from the surface (Figure 3.8(a)), increasing drag and decreasing lift. However, as seen in Figure 3.8(b), the turbulent boundary layer will tend to stick to the surface for a longer period prior to separating [20,31]. Therefore, it is important to trip the boundary layer to maintain dynamic similarity.

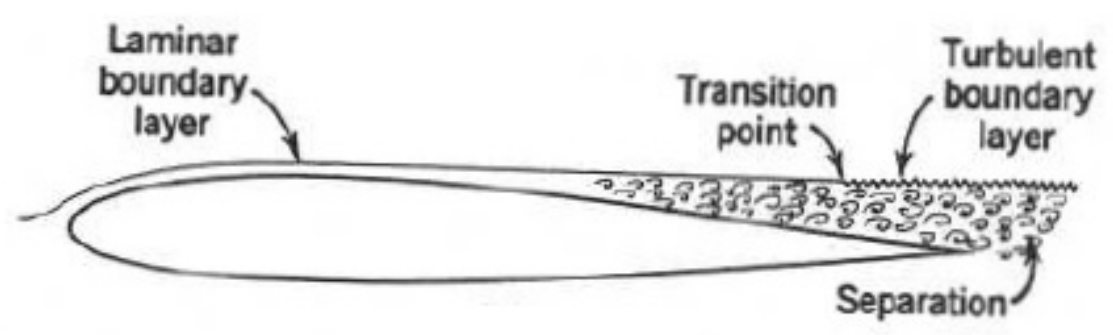

(a)

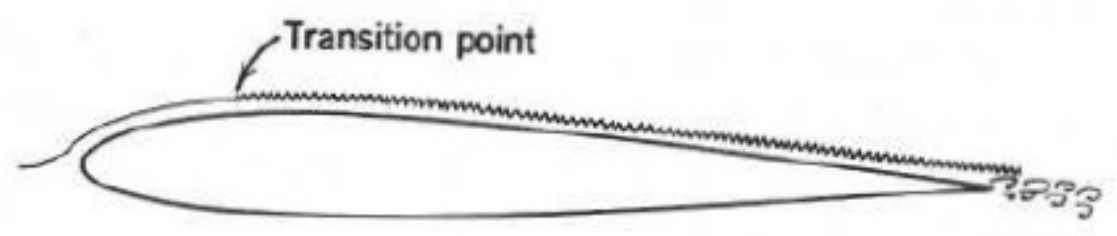

(b)

Figure 3.8: Representation of (a) laminar boundary layer and (b) transitional boundary layer $[20]$.

Types of trip strips include grit, two- or three-dimensional tape, wire, and epoxy dots. The height of the trip strip, $h$, is approximated by the following equation [20]:

$$
h=\frac{12 K}{R e_{f t}}
$$


where $K$ is a non-dimensional condition at the top of the roughness particles. For Reynolds numbers greater than $100000, K$ is set to 600 [20]. $R e_{f t}$ is a Reynolds number per foot value. From Equation 3.5, a grit size of 0.0937 in (comparable to No. 10 grit) was chosen for the experiment. The appropriate grit size for these experiments is relatively large compared to the thickness of the wing and will increase the overall drag. The impact of the grit size is illustrated in Figure 3.9 20].

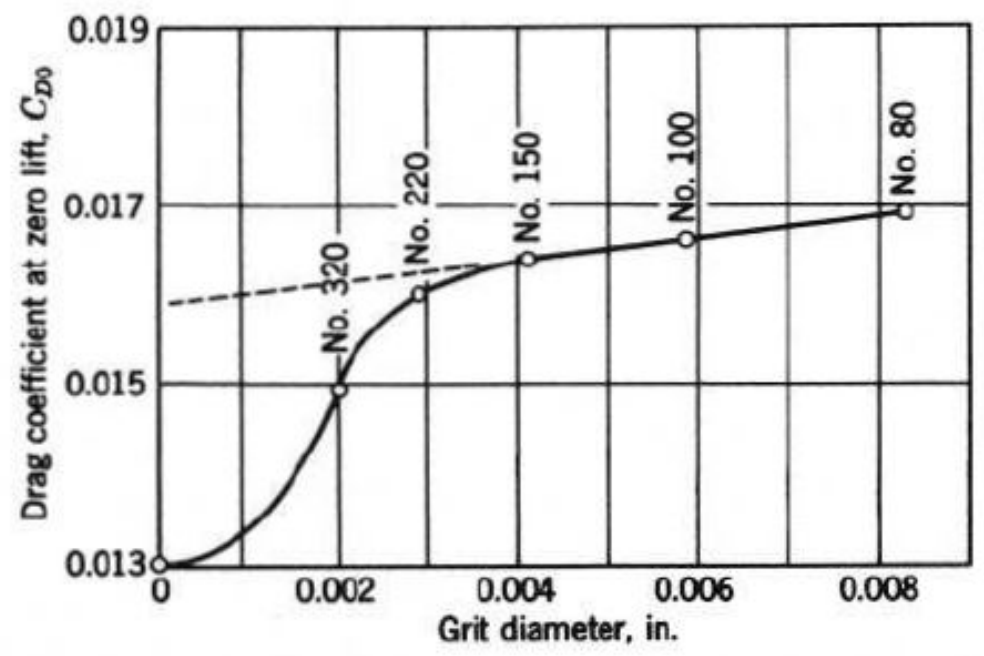

Figure 3.9: Change in drag coefficient with respect to a change in grit size [20].

The effect of the grit is quite substantial for small additions of grit on a clean wing. The addition of 0.002 in. of grit to a clean wing may increase the parasitic drag coefficient by $15 \%$. It can be observed that, at a grit size of approximately 0.004 in., the change in drag coefficients becomes linear and quite small. By extrapolating in the linear region to the grit size selected for these experiments, a $c_{D_{0}}$ value of 0.026 was obtained. For stability experiments, the impact of the grit size will not be significant whereas any performance analysis will require an accurate correction for grit size [20]. In these experiments, the drag effects of the trip strip where briefly analysed by running an experiment on the model with and without the trip strip. 
Moreover, the chord-wise location of the trip strip is also important. When placing a trip strip on a model, it should be strategically placed in order to match the transition point that is commonly found on the full scale model. Unfortunately, it is difficult to determine the exact point at which transition will occur. For the wing, a common approximation is to place the trip strip at $10 \%$ chord [20]. For a fuselage, it is approximately at $5 \%$ of its length $[20]$. The ultra-light wing with the trip strip added is seen in Figure 3.10 .

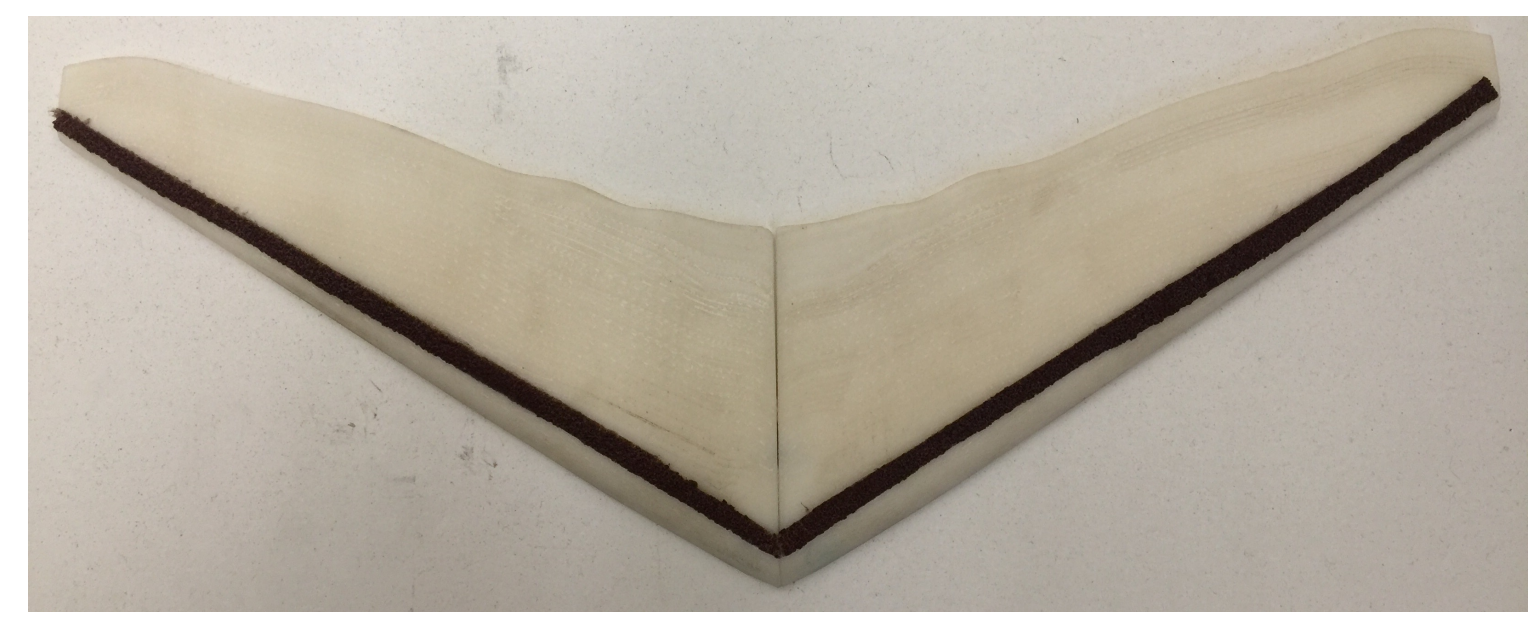

Figure 3.10: Ultra-light wing model with the addition of a 3-dimensional trip strip.

It should be noted that the transition point will occur at different chordwise locations depending on its angle of attack and Reynolds' number. A more representative method of deciding on the location of the trip strip is the application of Thwaites' method for laminar boundary layer assessment [35]. It is an approximate solution to the momentum integral equation (Equation 3.6) for low-speed flows. Information about the shear force, $\tau_{w}$, along with the momentum thickness, $\theta$, and displacement thickness, $\delta^{*}$ in the boundary layer are obtained [36] through this solution.

$$
\frac{d \theta(x)}{d x}+2\left(\theta(x)+\delta^{*}(x)\right) \frac{1}{U(x)} \frac{d U(x)}{d x}=\frac{\tau_{w}}{\rho U(x)^{2}}
$$

Although other approximate solutions to the momentum integral equation for a 
laminar boundary layer exist, Thwaites' method does not require any assumptions on the velocity profile (the Karman-Pohlhausen approximation is another type of approximation that requires the velocity profile to be expressed as a 4th order polynomial [37]). The general solution for the momentum thickness given a velocity profile for Thwaites' method is as follows [36]:

$$
\theta(x)^{2}=\frac{1}{U(x)^{6}}\left[U(0)^{6} \theta(0)^{2}+0.45 \nu \int_{0}^{x} U(x)^{5} d x\right]
$$

Once the momentum thickness is determined, a linear empirical relationship exists in which the displacement thickness and wall shear can be determined [36]. This can be used to solve for the location at which the laminar boundary layer separation occurs (i.e. at $\tau_{w}=0$ ). This location would be more suited for a trip strip but it will also vary due to the variation in the velocity profile as the angle of attack increases.

\subsection{Model Support}

The wind-tunnel balance is installed above the test section (as seen in Figure 3.7) and therefore the model must be mounted upside-down in order to avoid disturbing the suction side of the wing [38]. The locations on the model to which rods connect the model to the external balance are referred to as pick-up points. On the wing-only model, due to its thickness, it is difficult to include pick-up points on the lower surface of the wing without significantly changing the geometry of the wing. For this reason, an approach similar to the wing and fuselage combination was taken. Rather than incorporating a fuselage to the model, a sphere was used as seen in Figure 3.11 and 3.12 . 


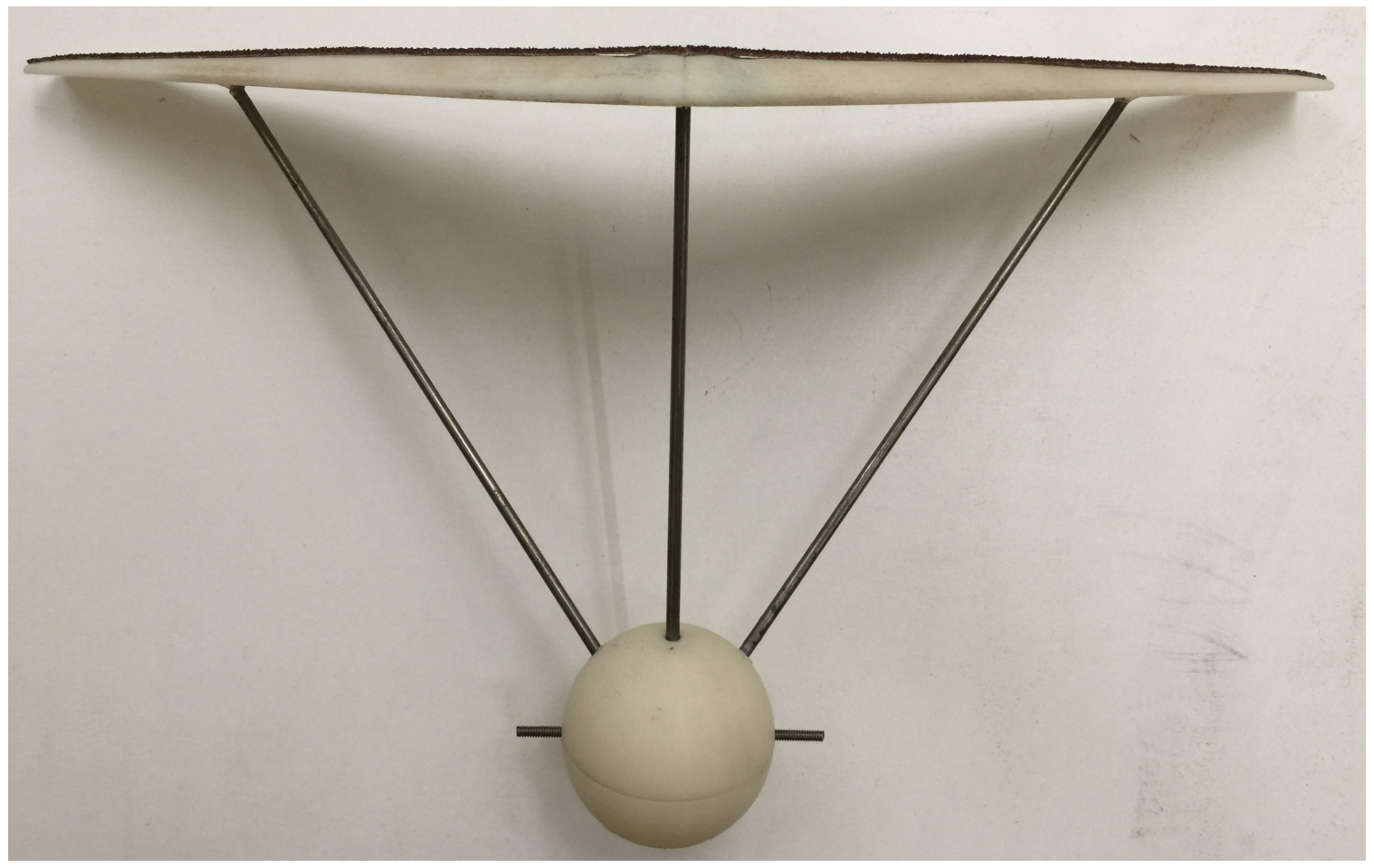

Figure 3.11: Ultra-light wing and sphere configuration

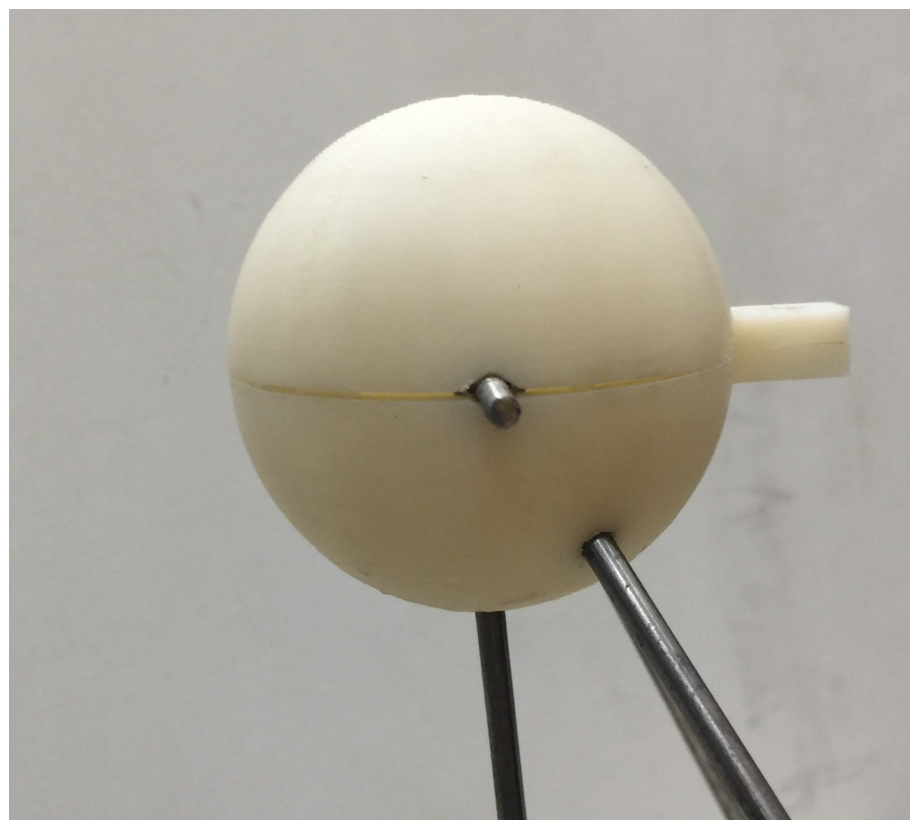

Figure 3.12: Sphere used for wing-only experiments 
The sphere is mounted at a distance $13 \mathrm{~cm}$ from the lower surface of the wing. This distance was chosen in order to reduce the aerodynamic interaction between the wing and the sphere while keeping both bodies far enough from the test section walls to avoid wall effects. In doing so, the aerodynamic load contribution from the sphere may be accounted for and removed when analysing the wing's aerodynamic characteristics in isolation. For both the wing-only and ultra-light model, the design considered 3 points of attachment to the external balance. Steel 4-40 rods connected the model to the external balance at all pick up points; the configuration of the external balance influenced the decision to use these type of rods.

\subsection{External Balance}

The aerodynamic loads were measured using a 3-component external mechanical balance that had been retrofitted with 3 single-axis load cells (Figure 3.13). The load cells convert an applied force into an electrical signal. The balance is designed to limit the load paths to three directions: axial, normal, and moment about a pivot point. Therefore, it is important to ensure that the balance axes are parallel to the wind-tunnel axes in order to accurately record the aerodynamic loads. Prior to running any experiment, the balance is leveled.

There are three sets of prongs connected to the balance that are grouped and color coded. Each set records a force in one direction:

- Red/blue prongs measure axial force

- Orange/green prongs measure normal force

- Black/white prongs measure force about the pivot point 


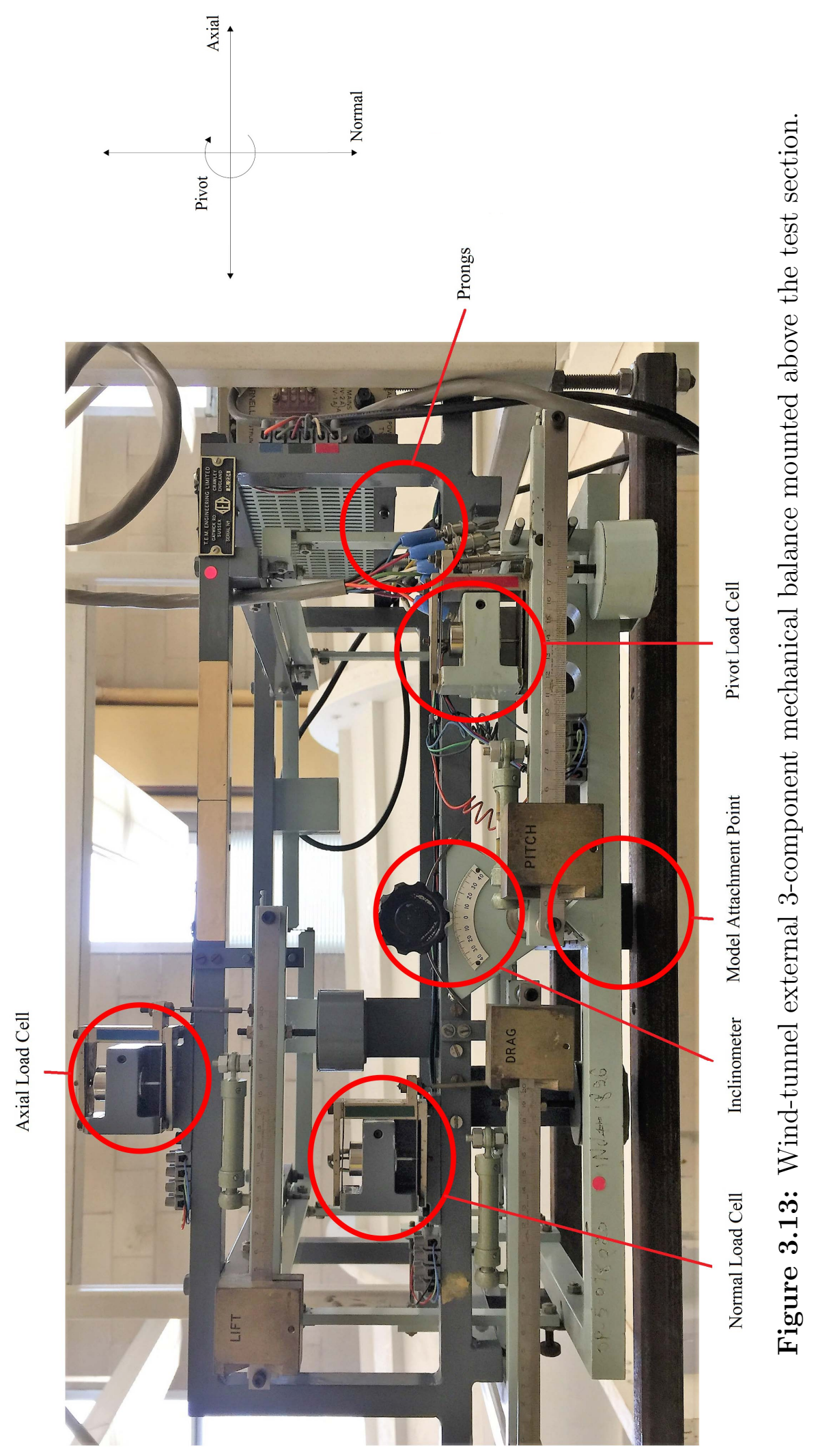




\subsubsection{Calibration of Load Cells}

All load cells were calibrated individually. The balance was strategically loaded in a manner in which the applied load is only recorded by a single load cell. For instance, when measuring the load normal to the flow in the wind-tunnel axes, weights were incrementally hung from the crossbar. As weights were added, the change in voltage was recorded. When measuring the the loads parallel to the flow, aircraft cable was tied to this cross bar and wrapped around a pulley. A level (accuracy of $\pm 0.5^{\circ}$ ) was used to ensure that the cable was aligned with the axis that is parallel to the flow. Weights were then suspended from the exposed end of the cable and measurements were taken. Finally, the load cell that measures the force about the pivot point was calibrating by suspending weights on the moment arm at distance of $13 \mathrm{~cm}$ from the pivot point. As each individual load cell was being calibrated, the voltage readings on the other load cells were also being monitored. A linear equation was developed that relates the applied load to the voltage reading for each load cell (See Appendix C). Due to the nature of the balance, there is some coupling between all load cells. From the calibration results, it is clear that the variation in the voltage output is linear for all load cells. Therefore, a system of linear equations is necessary to account for the coupling. The recorded loads, $F_{r}$, can be expressed as a function of the applied loads, $F_{a l}$, defined below 20]:

$$
\left[\begin{array}{c}
F_{r, N} \\
F_{r, A} \\
F_{r, M}
\end{array}\right]=\overbrace{\left[\begin{array}{lll}
C_{F_{r, N} F_{a l, N}} & C_{F_{r, N} F_{a l, A}} & C_{F_{r, N} F_{a l, M}} \\
C_{F_{r, A} F_{a l, N}} & C_{F_{r, A} F_{a l, A}} & C_{F_{r, N} F_{a l, M}} \\
C_{F_{r, M} F_{a l, N}} & C_{F_{r, M} F_{a l, A}} & C_{F_{r, N} F_{a l, M}}
\end{array}\right]}^{\text {calibration matrix }}\left[\begin{array}{l}
F_{a l, N} \\
F_{a l, A} \\
F_{a l, M}
\end{array}\right]
$$


where the subscripts $N, A$, and $M$ are the refer to the normal, axial, and moment load cells respectively. Every component of the calibration matrix, $C$, is the rate of change of the recorded load with respect to the applied load, i.e.:

$$
C_{F_{r} F_{a l}}=\frac{\Delta F_{r}}{\Delta F_{a l}}
$$

When calibrating each load cell individually, the applied load in the other directions will be zero. Therefore, the calibration matrix coefficients are developed columnby-column. One the calibration is complete, the calibration matrix is inverted and the applied aerodynamic loads can be calculated from the recorded output:

$$
\left[F_{a l}\right]=[C]^{-1}\left[F_{r}\right]
$$

Refer to Appendix C for the calibration matrix and calibration curves.

\subsection{Instrumentation}

\subsubsection{Inclinometer}

An inclinometer is included on the balance; it adjusts the angle of the moment arm. It is crucial that the rod that links the balance's moment arm to the model be stiff and not subject to bending in order to accurately measure the aircraft's angle of attack

or sideslip angle. As seen in the figure below, the inclinometer limited to angles of \pm $40^{\circ}$ but test section restrictions reduce the maximum angle significantly. 


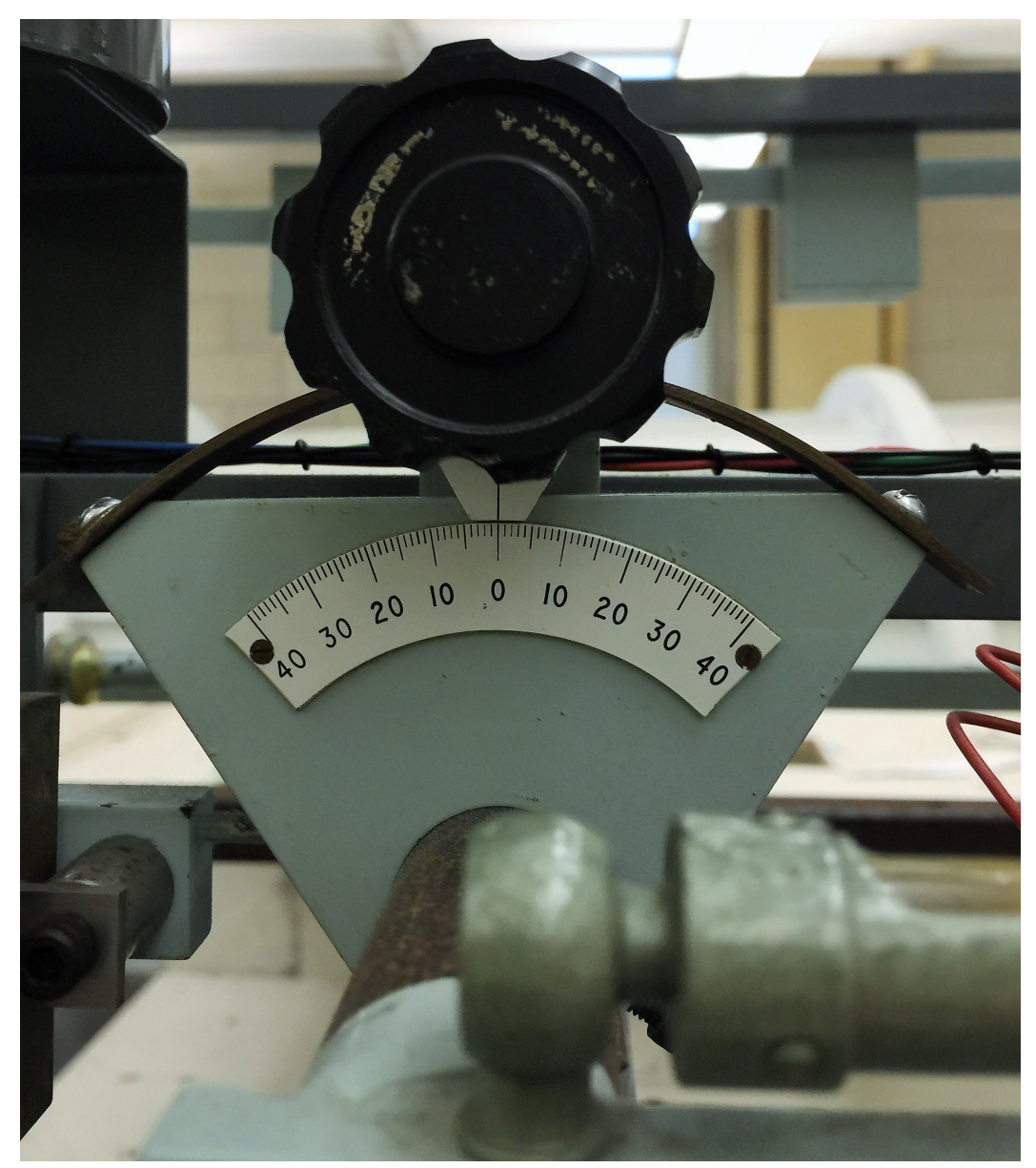

Figure 3.14: Inclinometer on the external balance.

\subsubsection{Thermometer}

The temperature near the outlet of the contraction is measured by a simple thermometer as seen in Figure 3.15(a). The gauge shown in Figure 3.15(b) displays the contraction outlet temperature in degrees Fahrenheit. During experimentation, the wind-tunnel air temperature can increase by $10^{\circ} \mathrm{F}$ to $15^{\circ} \mathrm{F}$. 

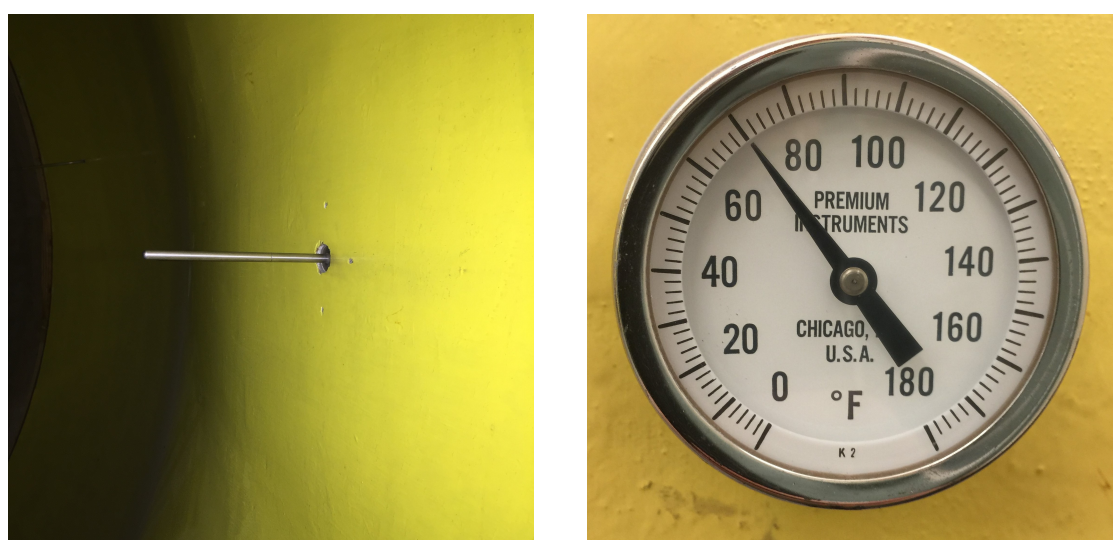

(a)

(b)

Figure 3.15: (a) Thermometer and (b) temperature gauge in the wind-tunnel contraction.

\subsubsection{Pressure Measurement Device}

The wind tunnel contraction pressure difference was measure by the use of a pitot tube, a static tap, and a U-tube manometer. A pitot tube is located at the inlet of the contraction and a static tap is located at the outlet of the contraction as seen in Figure 3.16, A rubber hose is connected between a U-tube manometer and the pitot tube. A second rubber hose is connected between a U-tube manometer and the static tap. The calculated pressure difference yields the dynamic pressure at the end of the contraction. It is assumed that the total pressure in the system is atmospheric and remains unchanged. It is also assumed that the dynamic pressure within the test section is the same as the dynamic pressure at the end of the contraction as there are no changes to the cross-sectional area 10 . 

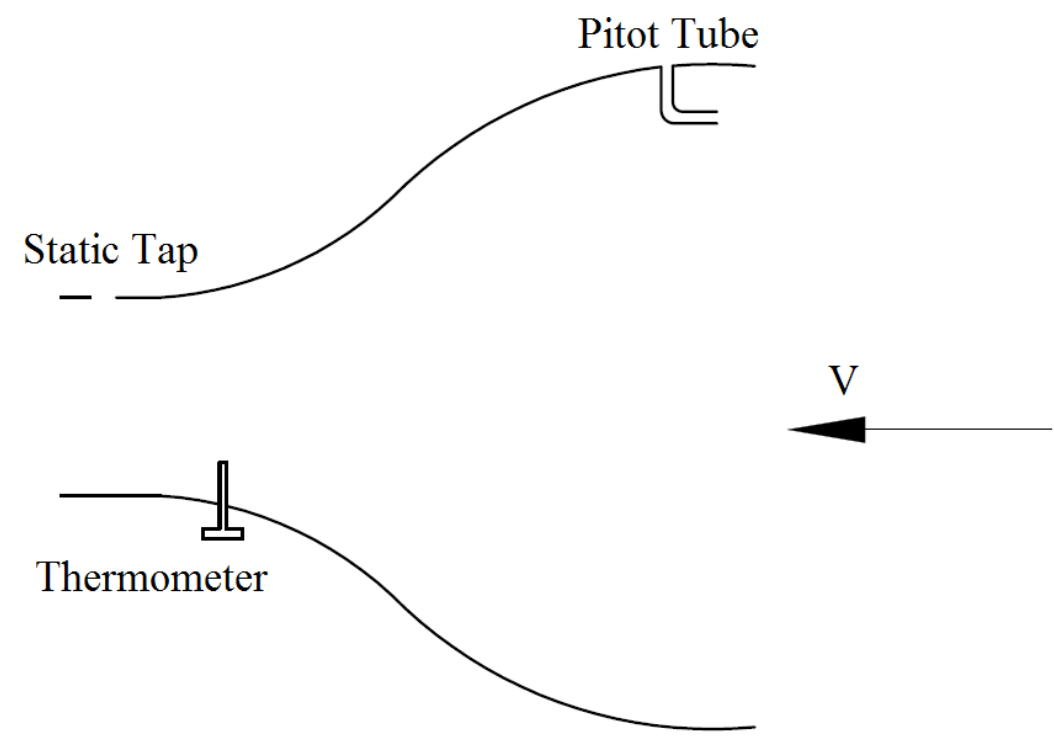

Figure 3.16: Schematic of the wind-tunnel contraction (not to scale).

\subsubsection{Data Acquisition}

The time-averaged DC voltage signals were read from a Fluke-179 True RMS Digital Multimeter. The specifications of the multimeter are found in Table 3.3 .

Table 3.3: Fluke-179 True RMS Digital Multimeter specifications 39].

\begin{tabular}{|l|c|}
\hline Range & $\pm 6.000 \mathrm{~V}$ \\
\hline Resolution & $0.001 \mathrm{~V}$ \\
\hline Accuracy* & $\pm[0.09 \%$ of reading +2 counts $]$ \\
\hline
\end{tabular}

* When taking an average reading of DC functions, the accuracy is $0.09 \%+12$ counts 


\subsubsection{Wind-Tunnel Commissioning Experiments}

Once the test section was built and the load cells were calibrated, experiments were run to determine the flow velocity and uniformity in the test section. An initial experiment was run where a pitot-static probe was set up at the centre of the test section. This probe was connected to a U-tube manometer using rubber hoses. A second U-tube manometer was connected to the pitot tube and static tap at the inlet and outlet of the contraction, respectively. The change in manometer height was recorded at both the centre of the test section as well as across the contraction for various fan frequencies; the wind-tunnel velocity was then calculated from these manometer readings. From Figure 3.17, it can be observed that the velocity calculated at the outlet of the contraction does not correspond to the velocity within the test section. The difference becomes more apparent as the velocity increases; the measurements from the contraction (marked as o's in the graph) begin lagging the measurements from the pitot-static probe (marked as x's) for similar fan frequencies. 


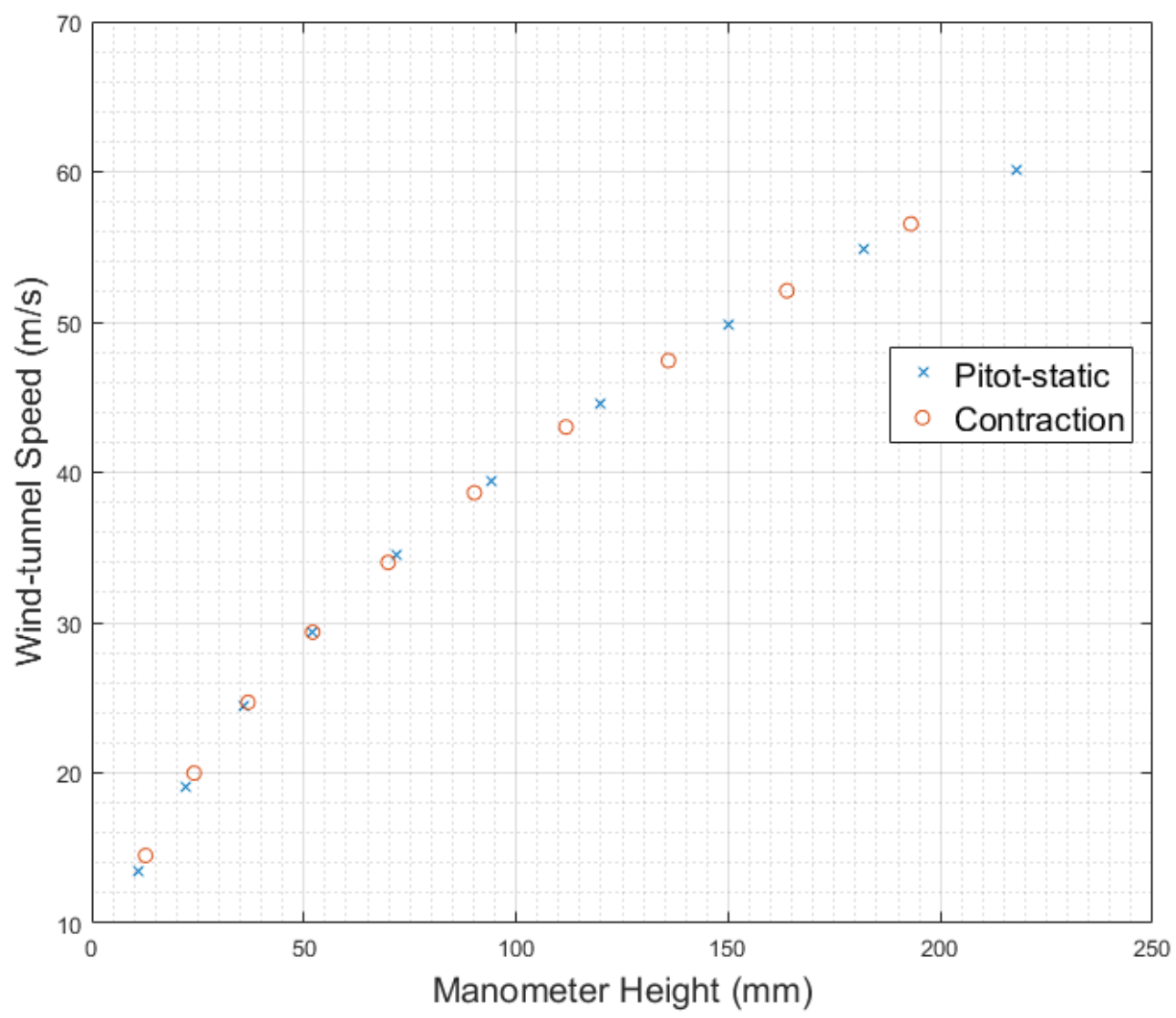

Figure 3.17: Velocity correlation between the pitot-static probe and the contraction pressure difference.

The velocity measured by the pitot-static probe at the centre of the test section is assumed to be the most representative for any experiment due to all models being mounted in this location. However, the pitot-static probe will not be inserted into the test section during the experiments. Alternatively, the wind-tunnel velocity will be measured from the contraction pressure difference. As seen in the figure above, the wind-tunnel velocity cannot be simply calculated from the contraction pressure difference due to the inaccuracy of the results. Thus, a corrected manometer height difference was introduced by correlating the manometer height from the pitot-static probe to that of the contraction. This correlation can be seen in Figure 3.18. The 
purpose of removing the pitot-static probe from the test section is to avoid obstructing the air flow.

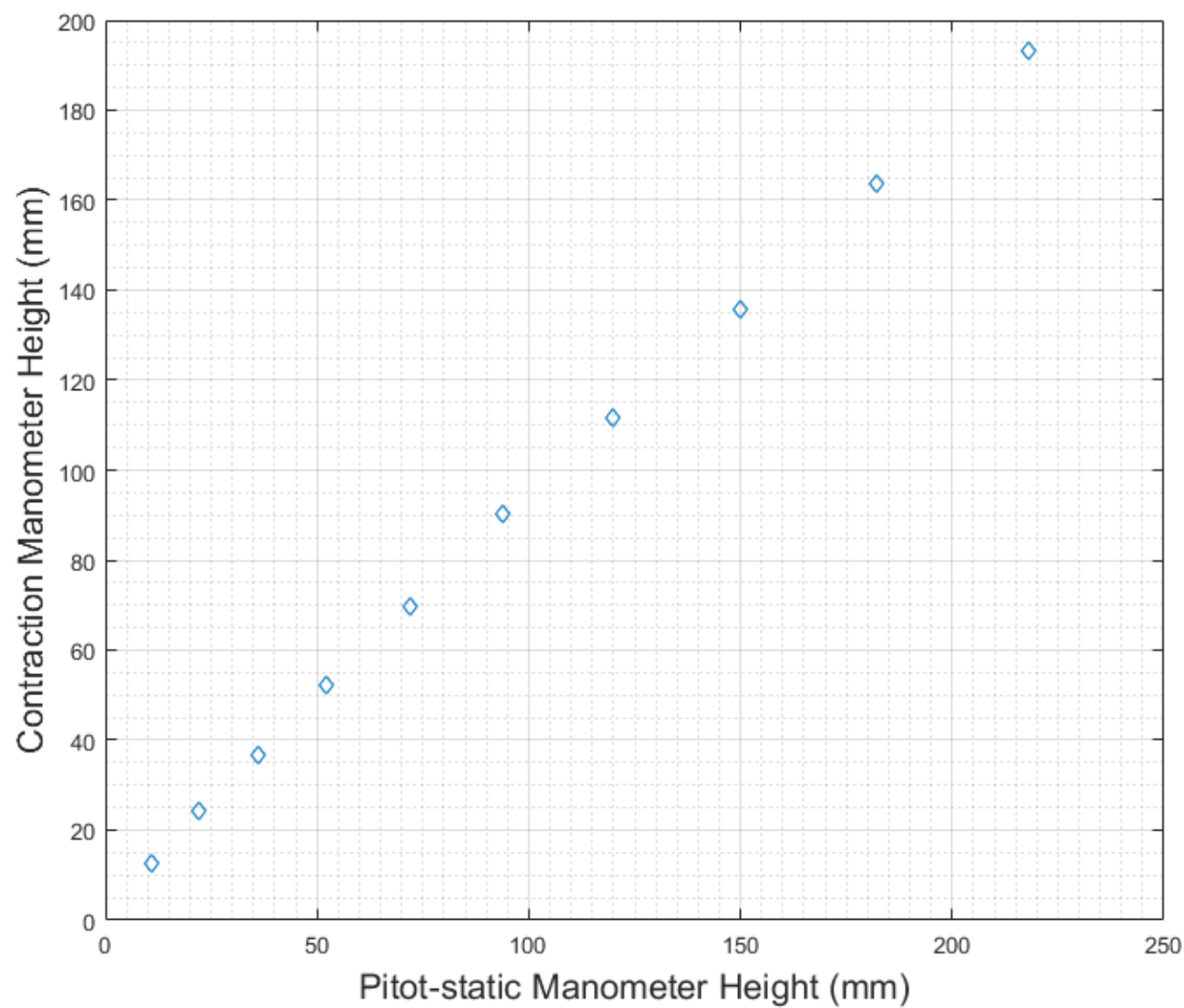

Figure 3.18: Manometer height correlation between the pitot-static probe and the contraction pressure difference.

A second experiment was undertaken in order to determine the flow uniformity in the test section. A pitot-static probe was placed at the centre of the test section and velocity readings were taken across its height. The normalized velocity profile is shown in Figure 3.19. From this figure, it is clear that during any point in the experiment, the model should not come within $7.6 \mathrm{~cm}$ of the upper or lower surface of the test section. Any velocity differences measured within the permissable range were within $1 \%$ of each other. 


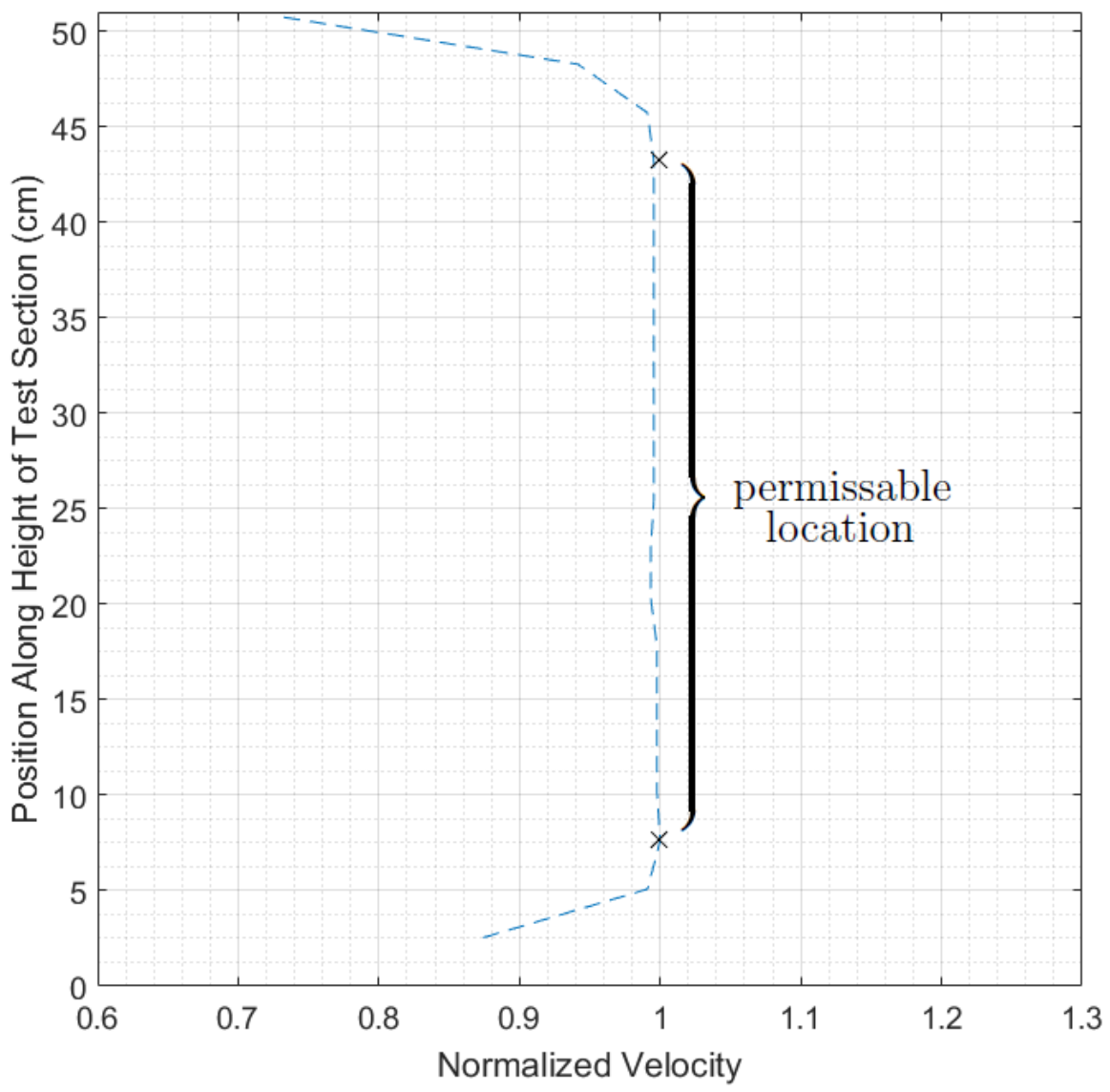

Figure 3.19: Velocity profile along the height of the test section.

During the experiments, the pitot-static tube's alignment with respect to the freestream air was continuously monitored. However, any probe misalignment effects were neglected as they do not have a significant impact on the measurements. If the probe is slightly misaligned $\left( \pm 3^{\circ}\right)$, the pitot tube will remain insensitive [20]. The static taps on the probe will be subject to erroneous readings at a $5^{\circ}$ misalignment. For these misalignments, the measurement error between $1 \%$ to $3 \%$ 22.

Due to the test section being purpose-built for these experiments, upon completion of the load cell calibration, a wind-tunnel validation test was undertaken on a 
circular cylinder mounted perpendicular to the flow. The purpose of this test was to commission the test section as well as to validate the calibration of the load cells on the external balance. Flow over a circular cylinder is a classic problem that has been thoroughly studied since the early stages of aerodynamic theory. There is an abundance of published experimental data on aerodynamic loads over a circular cylinder therefore it is favorable choice for a commissioning test [10].

\subsection{Experimental Uncertainties}

There are sources of uncertainties present in all instrumentation used during experimentation; they can be systematic or random. The systematic uncertainties are referred to as bias errors and the random uncertainties are said to be precision errors [22]. The bias error in the instrumentation is quantified in Table 3.4 below.

Table 3.4: Quantification of the bias error in the instrumentation.

\begin{tabular}{|c|c|}
\hline Instrument & Uncertainty \\
\hline Manometer & $\pm 1 \mathrm{~mm}$ \\
Thermometer & $\pm 1^{\circ} \mathrm{F}$ \\
Multimeter & $\pm[0.00009 \cdot V+0.012] V$ \\
Barometer & $\pm 0.05 \mathrm{in} . \mathrm{Hg}$ \\
Inclinometer & $\pm 0.5^{\circ}$ \\
\hline
\end{tabular}

Precision errors are random and always present within a system. These errors become apparent when a measurement is repeated and the results differ. Thus, they are more difficult to quantify. It can be assumed that a repeated measurement of the 
same quantity will yield a normal distribution and that the mean of this distribution can be used to estimate the estimate the true value [22]. With this assumption, the precision error may be expressed as 2 standard deviations from the mean. The total uncertainty in the system can be defined as 22$]$ :

$$
u=\sqrt{b^{2}+p^{2}}
$$

where $b$ and $p$ are the bias and precision errors respectively. When a parameter is computed by the use of various direct measurements, the bias of each measurement must be considered. For example, the the lift coefficient, $c_{L}$, is a function of several measured quantities, $x_{n}$ (i.e. $\left.c_{L}\left(x_{1}, x_{2}, \ldots, x_{n}\right)\right)$. Therefore, the total bias in the lift coefficient can be calculated as [22]:

$$
b_{c_{L}}=\sqrt{\sum_{n=1}^{N}\left(\frac{\partial c_{L}}{\partial x_{n}} b_{x_{n}}\right)^{2}}
$$

Similarly, the precision of the lift coefficient can be determined by:

$$
p_{c_{L}}=\sqrt{\sum_{n=1}^{N}\left(\frac{\partial c_{L}}{\partial x_{n}} p_{x_{n}}\right)^{2}}
$$

\subsection{Test Matrix}

A test matrix was developed for both wing-only and the complete ultra-light aircraft model; they can be seen in Table 3.5 and 3.6 respectively. The results obtained from the wing-only test were to be used for comparative purposes with an existing CFD simulation. In regard to the complete ultra-light model, two test cases were examined in order to obtain results that will be used to develop the static stability derivatives. 
Table 3.5: Test matrix for the wing-only experiments.

\begin{tabular}{|c|c|c|c|}
\hline \multicolumn{4}{|c|}{ Case 1 } \\
\hline & Angle of Attack $(\alpha)$ & Reynolds Number & Measured Loads \\
\hline Without Trip Strip & $-4^{\circ}$ to stall & $2.0 \cdot 10^{5}-4.0 \cdot 10^{5}$ & $X, Z, M$ \\
\hline With Trip Strip & & & \\
\hline
\end{tabular}

Table 3.6: Test matrix for ultra-light model experiments.

\begin{tabular}{|c|c|c|c|}
\hline \multicolumn{4}{|c|}{ Case 1} \\
\hline & Angle of Attack $(\alpha)$ & Reynolds Number & Measured Loads \\
\hline Without Trip Strip & \multirow{2}{*}{$-5^{\circ}$ to stall } & \multirow{2}{*}{$2.0 \cdot 10^{5}-4.0 \cdot 10^{5}$} & \multirow{2}{*}{$X, Z, M$} \\
\hline With Trip Strip & & & \\
\hline \multicolumn{4}{|c|}{ Case 2} \\
\hline & Sideslip Angle $(\beta)$ & Reynolds Number & Measured Loads \\
\hline With Trip Strip & $0^{\circ}$ to $18^{\circ}$ & $2.0 \cdot 10^{5}-4.0 \cdot 10^{5}$ & $Y, N$ \\
\hline
\end{tabular}

\subsection{Experimental Procedure: Wing-Only}

\subsubsection{Test Case 1}

In the wing-only experiment, the only case that was studied was the change in lift, drag and pitching moment with respect to a change in angle of attack of the PROFI TL 14 wing. The experimental procedure for this case is listed below: 
1. The model is set up within the test section and mounted on the balance. The opening on the upper side of the test section is covered. A bubble level is used on the lower surface of the model to ensure that its angle of attack matches the angle of the inclinometer located on the external balance.

2. Prior to engaging the motor, the balance is switched on. Each set of prongs listed in Section 3.5 above must be successively connected to the multimeter and the zero-load measurement for all load cells must be recorded.

3. A barometer is used to determine the atmospheric pressure at the beginning of the experiment.

4. The motor is then engaged and the fan frequency is gradually increased to 50 Hz. Once the fan frequency reaches $50 \mathrm{~Hz}$, the flow is given sufficient time to reach steady state. When the change in manometer height settles, this is an indication that steady state has been reached.

5. Starting at an angle of attack of $-4^{\circ}$, the manometer height difference, windtunnel temperature, and voltage for each load cell are recorded. The multimeter setting should be placed on $A V G$ when recording voltages. For the base test, it is common practice to increase the angle of attack is in increments of $1^{\circ}$. For any consecutive tests, a $2^{\circ}$ angle of attack increase is acceptable [20].

6. At each angle of attack, the flow must once again be given sufficient time to reach steady state. The time-averaged voltage reading on the manometer would have settled or will fluctuate $1 / 1000$ of a volt; this is an indication that the flow has reached a steady state.

7. Once the experiment is complete, the model is returned to its original angle of attack, the motor is shut off and the zero-load voltage is re-taken to ensure that no drift has occurred. 


\subsection{Experimental Procedure: Ultra-light Model}

\subsubsection{Test Case 1}

Test case 1 for the ultra-light model follows the same procedures as for the wing-only experiment. The setup is seen in Figures 3.20 and 3.21 . The axes system shown in this figure are the wind tunnel axes. As previously stated, the model is mounted upside down due to the external balance being placed above the test section. Three rods connect the aircraft to the external balacne and the angle of attack changes by pivoting the aircraft about the two forward support rods. 


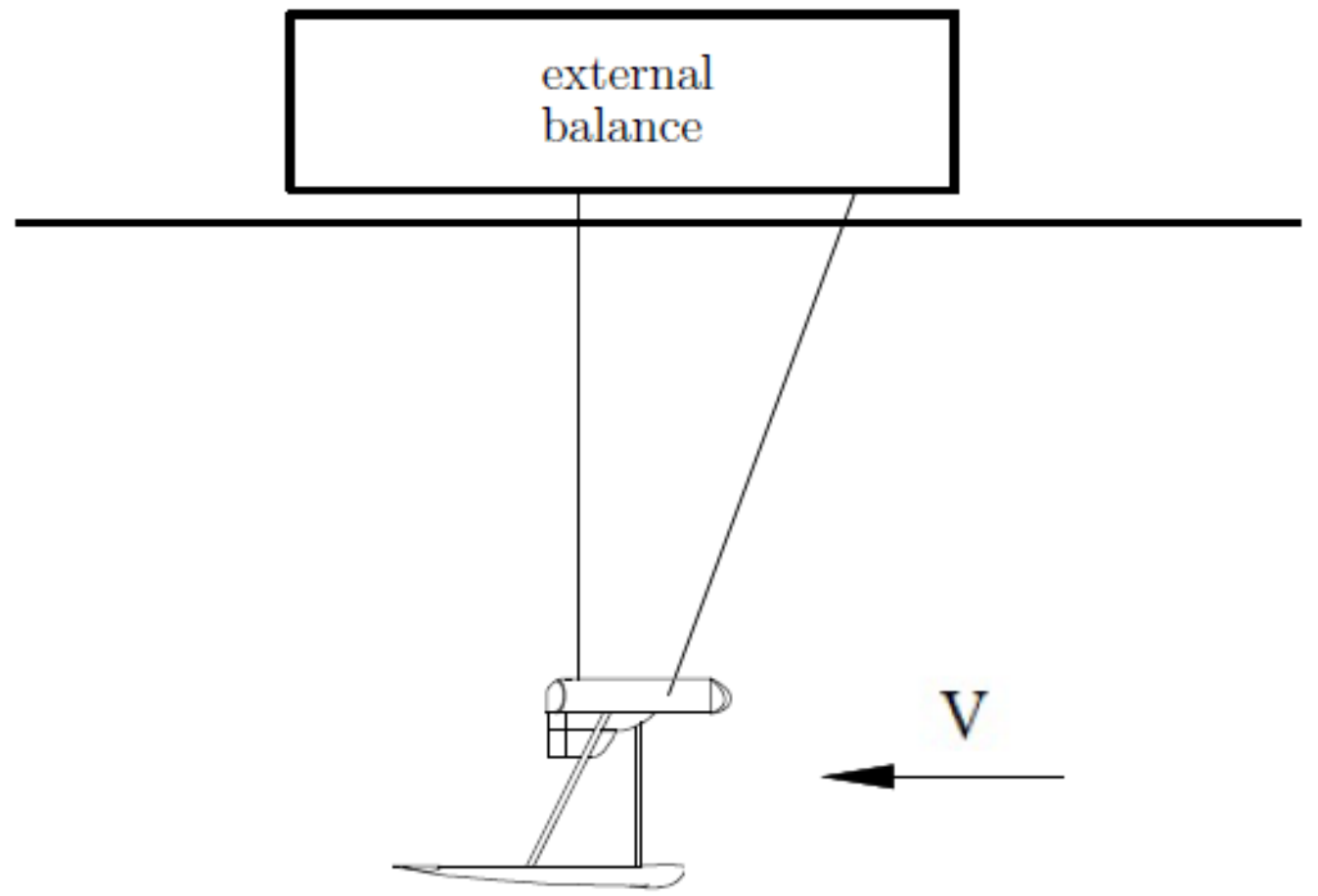

Figure 3.20: Representation of Test Case 1 with the model connected to external balance. 


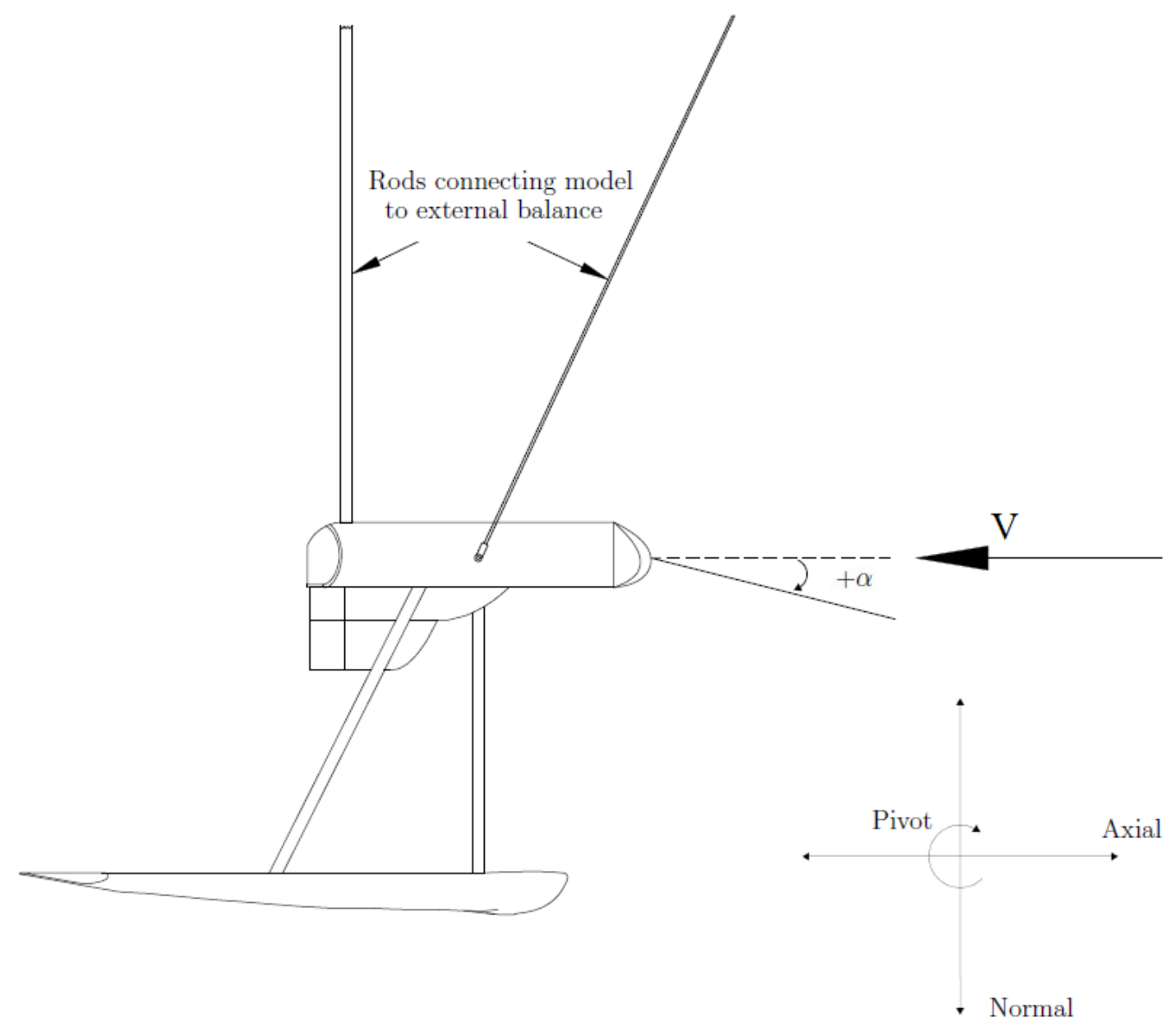

Figure 3.21: Representation of Test Case 1 with respect to the wind-tunnel axes. 


\subsubsection{Test Case 2}

As seen in Table 3.6, the objective of the second test case was to extract the lateral force and yaw moment of the model with respect to a change in sideslip angle. Due to the physical restrictions of the external balance, the model must be mounted on its side as seen in Figures 3.22 and 3.23 . Specifically, the plane defined by the bottom surface of the fuselage should be parallel to the test section plane defined by the axial and normal vectors (Figure 3.23). In doing so, the inclinometer can be effectively used to change the model's sideslip angle. The steps outlined above remain unchanged for

this test case as well. The only change in the procedure is in the 5th step. Due to symmetry, the side force and yaw moment measured at a positive or negative sideslip angle will have an equal and opposite magnitude. Therefore, the initial sideslip angle is set to $0^{\circ}$; the sideslip angle is then increased incrementally to $18^{\circ}$. At this sideslip angle, the wing tip of the aircraft approaches the test section's lower surface. The velocity profile is no longer uniform in this location and thus the sideslip angle should not exceed $18^{\circ}$. 


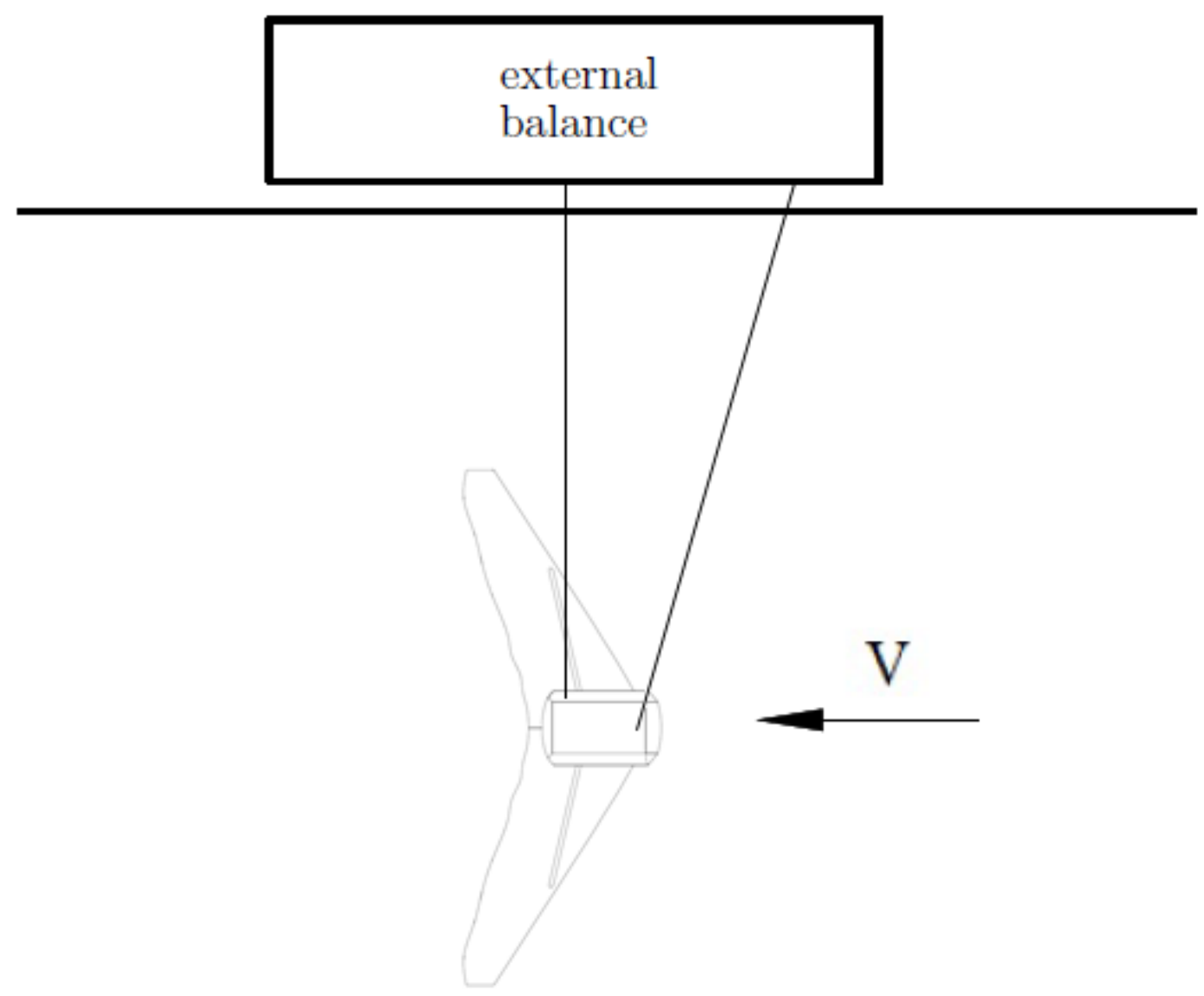

Figure 3.22: Representation of Test Case 2 with the model connected to external balance. 


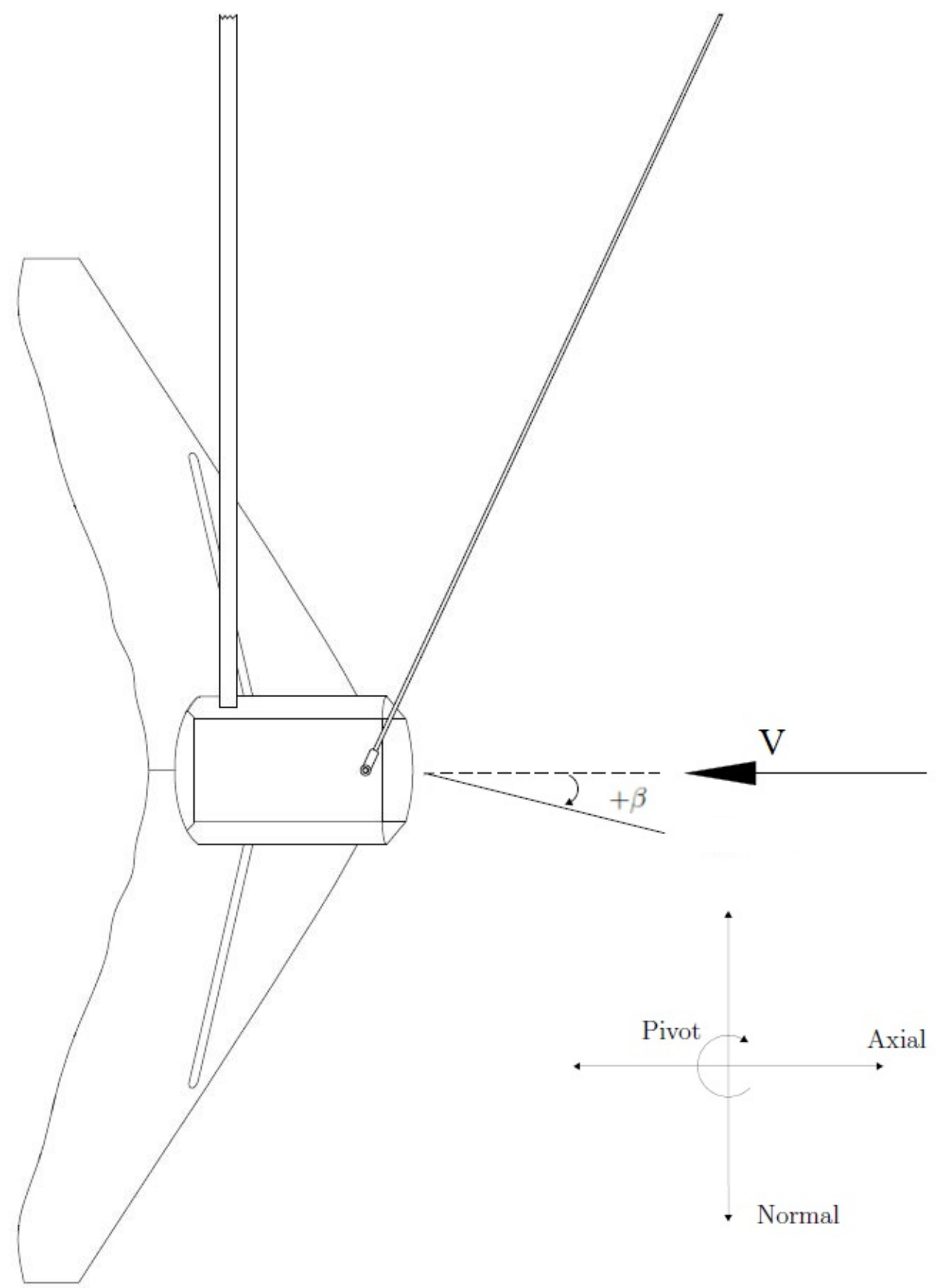

Figure 3.23: Representation of Test Case 2 with respect to the wind-tunnel axes. 


\subsection{Data Reduction}

In order to determine the non-dimensional coefficients required, there are a series of calculations required. The purpose of recording the wind-tunnel temperature and atmospheric pressure is to determine the air density. The density can be calculated from the ideal gas law [10]:

$$
\rho_{a i r}=\frac{p_{a t m}}{R_{a i r} T}
$$

where $R_{\text {air }}$ is the specific gas constant for dry air. The density is calculated for every data point due to the temperature increase in the test section over the course of the experiment. Following the calculation of the density, the freestream velocity in the test section is determined

$$
V=\sqrt{\frac{2 \rho_{\text {water }} g \Delta z_{\text {corr }}}{\rho_{\text {air }}}}
$$

where $\Delta z_{\text {corr }}$ is the corrected manometer height difference illustrated in Figure 3.18 When calculating a non-dimensional force coefficient, $c_{F}$, the equation is given as:

$$
c_{F}=\frac{F}{\frac{1}{2} \rho V^{2} S}
$$

The force, $F$, is a specific force measured by the balance. Equation 3.9 is used to determine this force component. 


\section{Chapter 4}

\section{Experimental Results and Discussions}

Prior to running any of the test cases that were outlined in the previous chapter, an experiment was undertaken in order to ensure that the experimental results that are measured are repeatable. Upon confirming the repeatability of the results, the experiments listed in the test matrices were completed. This chapter compares the results of the wing-only experiment to an existing CFD simulation. Furthermore, the longitudinal static stability in pitch and lateral static stability in yaw from the results obtained from the experiments of the ultra-light model is discussed.

\subsection{Repeatability of the Experiment}

Once the commissioning tests were complete, a repeatability test was undertaken on the ultra-light model as a whole without the trip strip. Test case 1 of the ultra-light

model experiment was used as the basis for the repeatability test. Figures 4.14 .3 illustrate the outcome of the tests. In Figure 4.1, the average percentage difference in the lift coefficient is approximately 5.5\%. In Figure 4.2 and 4.3 , these average differences are $6.6 \%$ and $5.7 \%$ respectively. If independent measurements are taken and found to be within a $10 \%$ difference, the measurement system is considered to be acceptable [40]. 


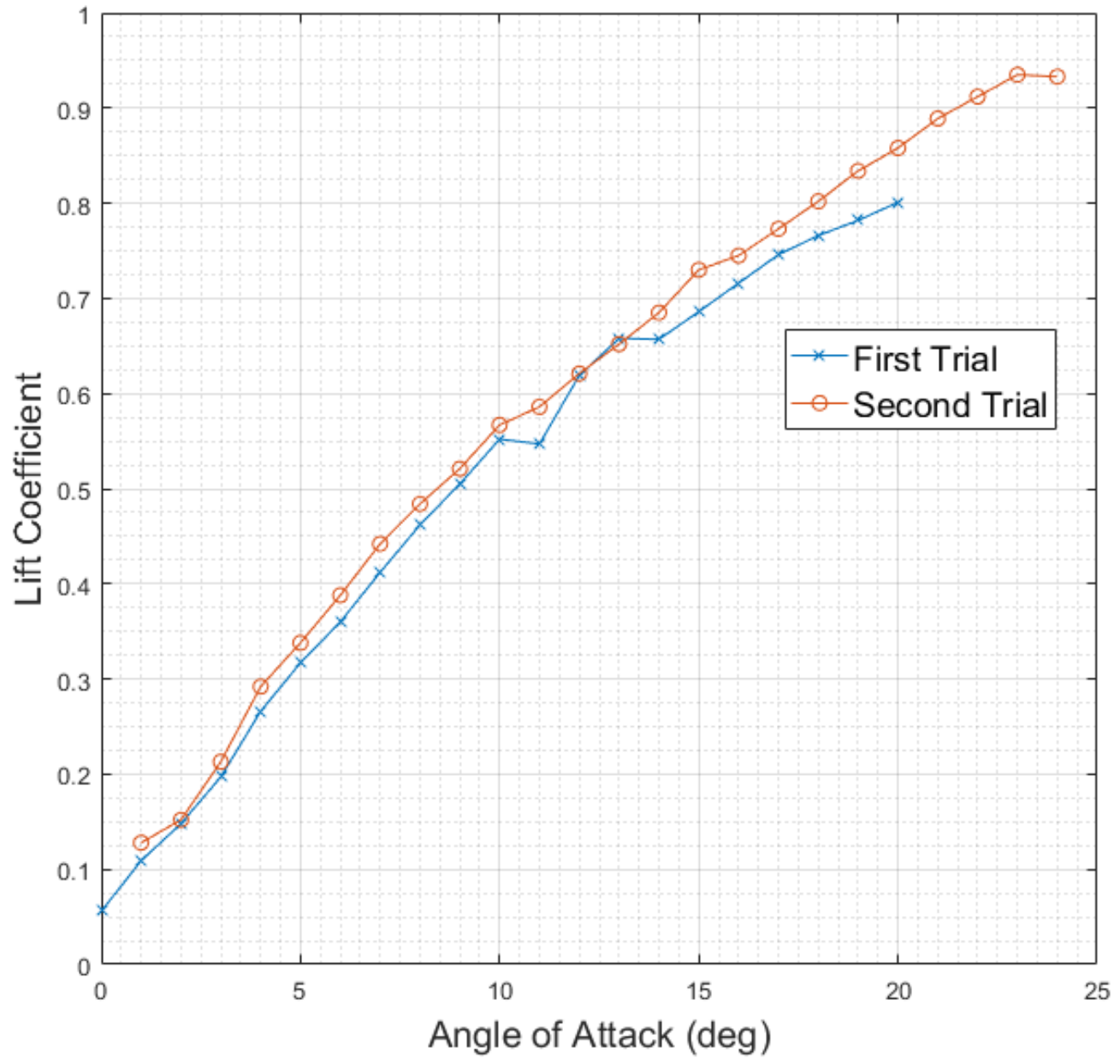

Figure 4.1: Repeatability test: $c_{L}$ vs. $\alpha$. 


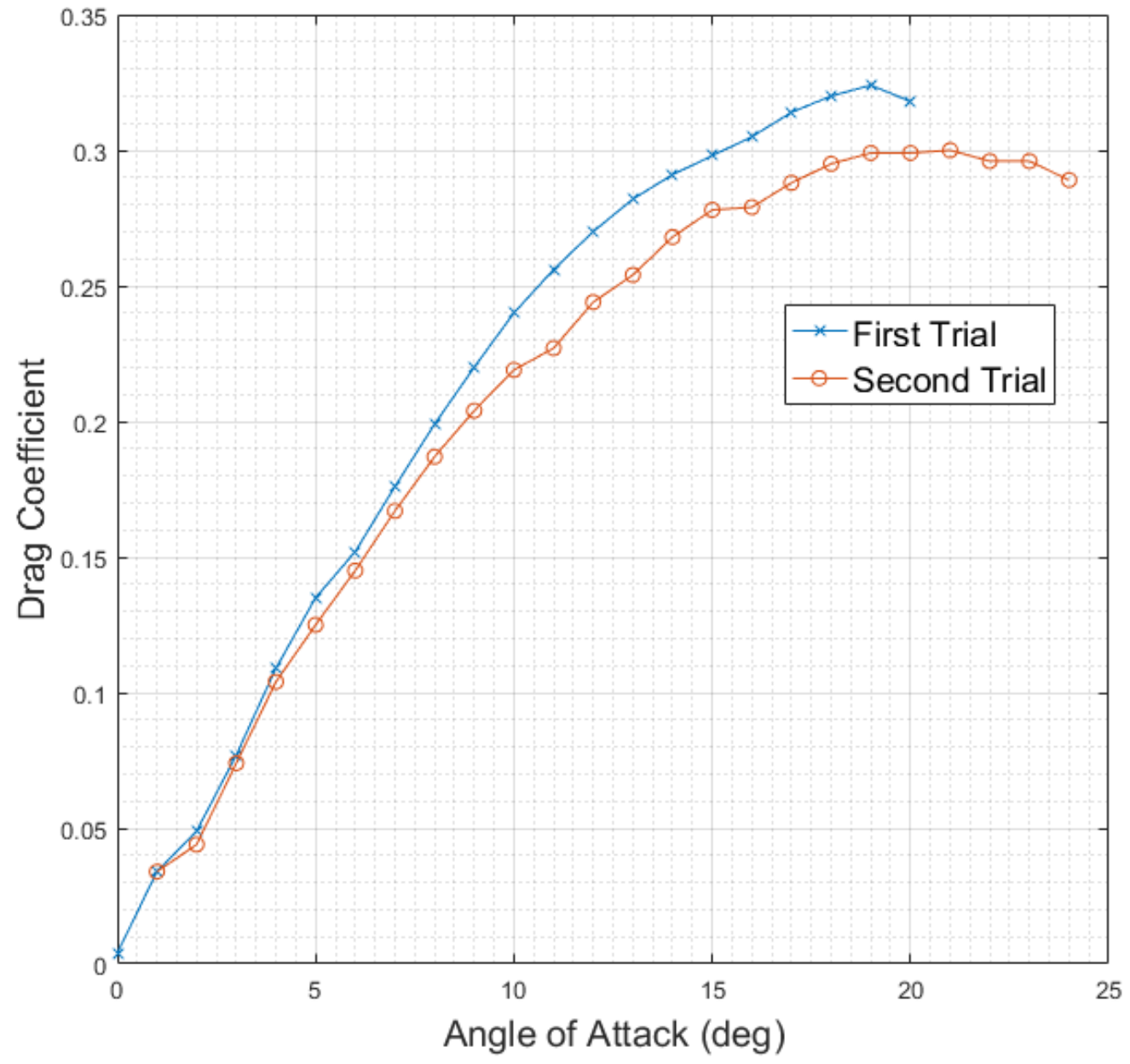

Figure 4.2: Repeatability test: $c_{D}$ vs. $\alpha$. 


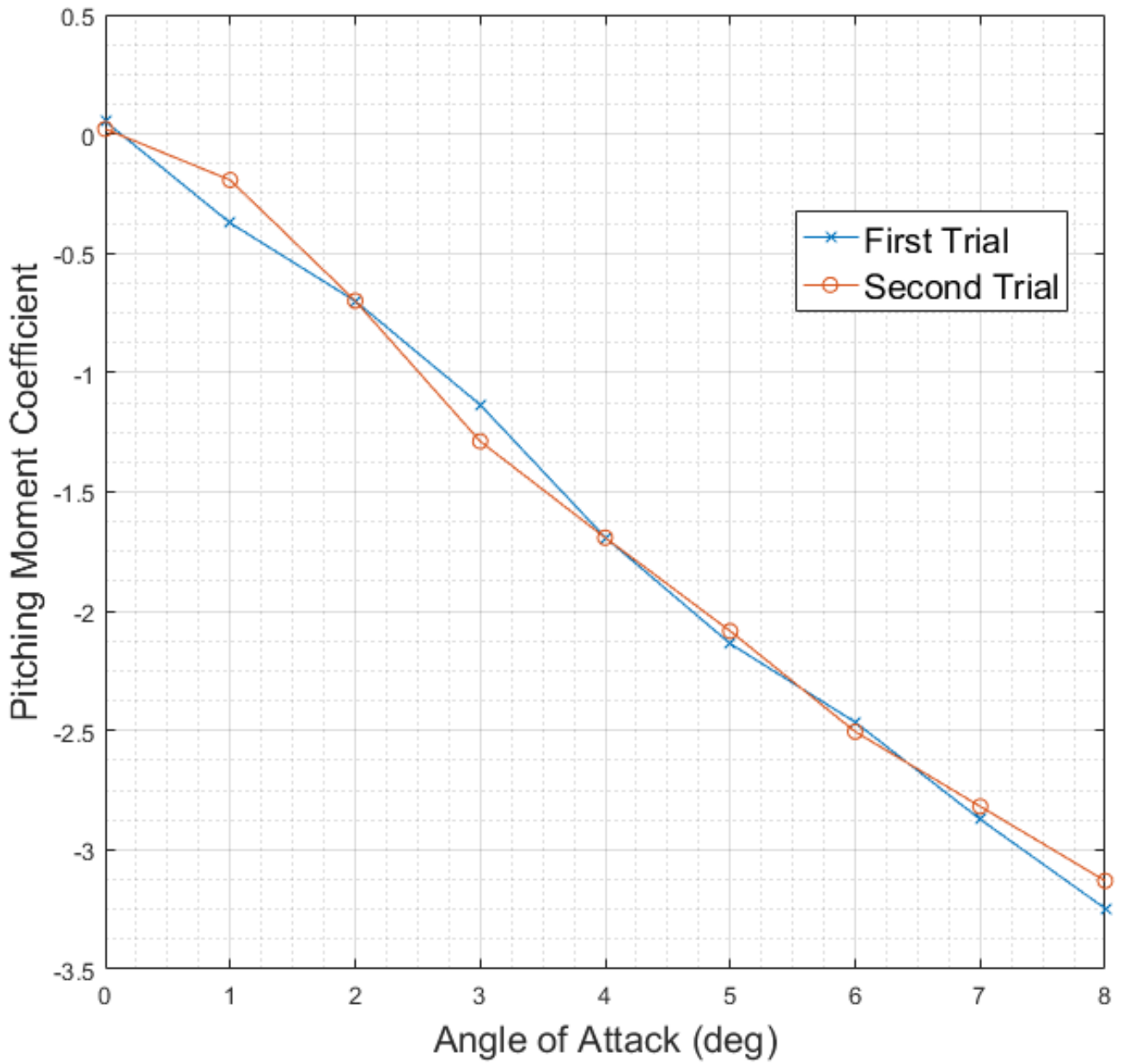

Figure 4.3: Repeatability test: $c_{M}$ vs. $\alpha$. 


\section{$4.2 \quad$ Wing-Only}

The only test case that was undertaken for the wing-only experiment was the measurement of the change in lift, drag, and pitching moment coefficients with respect to a change in angle of attack. The main focus of this experiment was to obtain results in order to make comparisons against an existing CFD simulation 27.

\section{Lift Coefficient}

Figure 4.4 illustrates the lift curve of the clean wing as well as the wing with a trip strip. The maximum lift coefficient for both the clean and tripped wing is 0.92 and 0.91 respectively ( $1.1 \%$ difference). The onset of stall on the clean wing begins at an angle of attack of approximately $18^{\circ}$ whereas it is seen to occur at $22^{\circ}$ on the tripped wing. The trip strip delays stall which is not commonly the case in experimentation at low Reynolds number. Traub tested various thicknesses of trip strips at various chordwise locations. It was found that tripping the boundary layer would demonstrate a notable reduction in lift as well as the onset of stall at lower angles of attack [32]. Specifically, lift reduction was observed at angles of attack greater than $2^{\circ}$ for a trip placed at $10 \%$ chord length from the leading edge [32]. Comparing this information to the results shown in Figure 4.4, a similar reduction in lift is apparent at an angle of attack of approximately $4^{\circ}$. Seddighi et al. also tested the effect a trip string on aircraft performance in a low speed wind tunnel. It was found that the roughness does not effect the linear region of the lift curve slope a lift reduction and stall delay are observed in many cases [34]. 


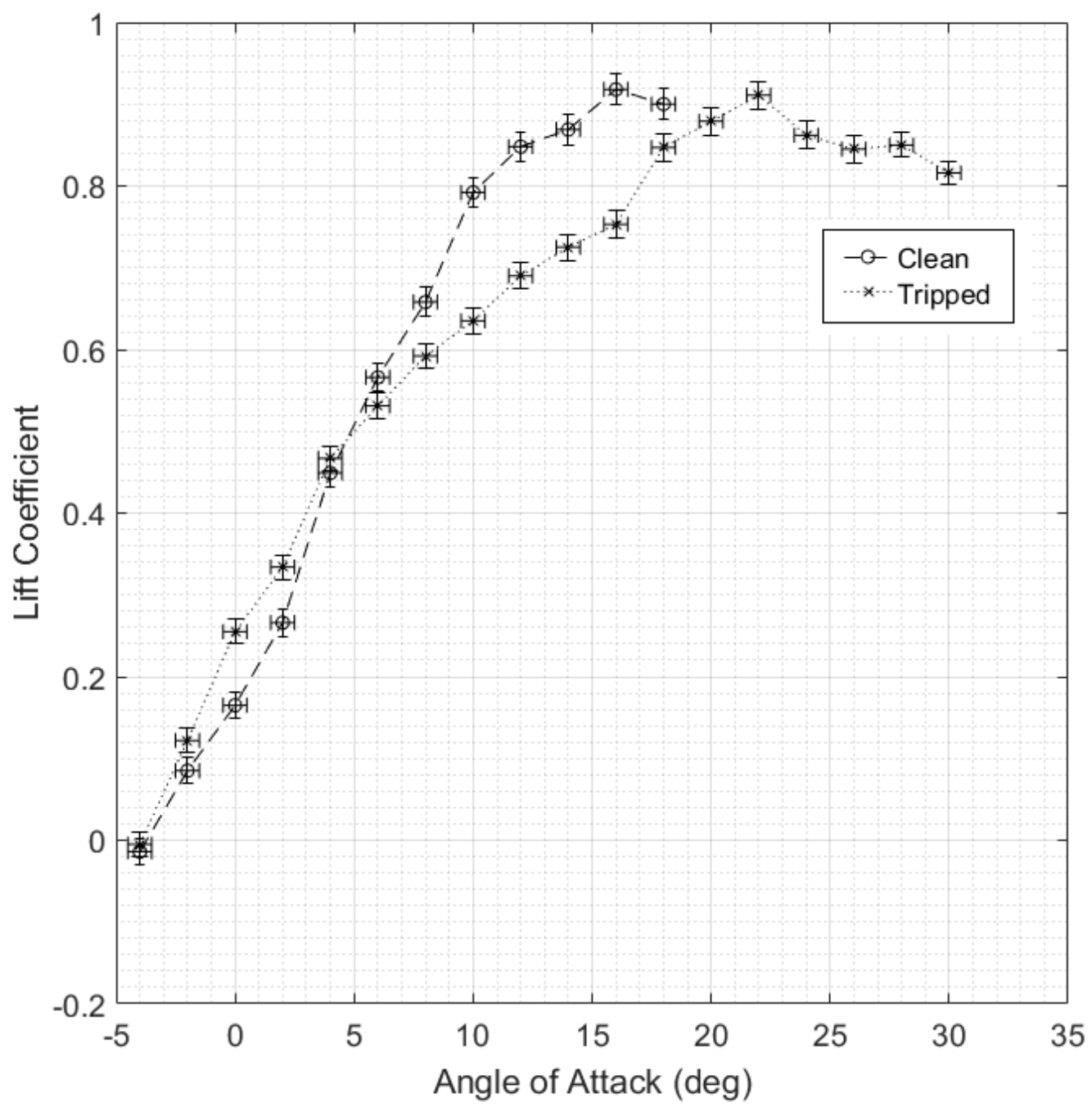

Figure 4.4: Wing-only test $-c_{L}$ vs. $\alpha$.

With regards to the stalling characteristics, low angle of attack stall is not a commonality in ultra-light wings. These types of wings can exceed angles of attack of $30^{\circ} 8,16,25,41$. The late onset of stall is likely due to the delay of boundary layer separation. For flow over a flat plate, boundary layer transition occurs at a critical Reynolds number of approximately $5 \cdot 10^{5}$; the critical Reynolds number is lower for smooth airfoils $\left(\sim 3 \cdot 10^{5}\right)[10,42$. The critical Reynolds number is also reduced as a consequence of surface roughness [10]. Therefore, at the Reynolds numbers 
tested during experimentation, the boundary layer on the wing may experience a late laminar-to-turbulent transition. In this case, boundary layer separation is likely to occur prior to transition and the onset of stall will become apparent. The trip strip will hinder the boundary layer separation on the wing by immediately causing laminar-to-turbulent transition. As discussed in Section 3.3, a turbulent boundary layer will separate further downstream as compared to a laminar boundary layer.

The gradient of the tripped wing within the linear region (at smaller angles of attack) is not as steep compared to the clean wing; a reduction in lift is expected for a tripped configuration [32]. The results of the CFD simulation were compared to both the clean and tripped results in Figure 4.5. The simulation was run to imitate real flight conditions listed below and it assumes fully turbulent flow throughout the entire domain. [27]:

- Freestream velocity: $45 \mathrm{~m} / \mathrm{s}$

- Density: $1.185 \mathrm{~kg} / \mathrm{m}^{3}$

- Dynamic viscosity: $1.8321 \cdot 10^{-5} \mathrm{~kg} / \mathrm{m} \cdot \mathrm{s}$

- Reynolds number: $4.9 \cdot 10^{6}$ 


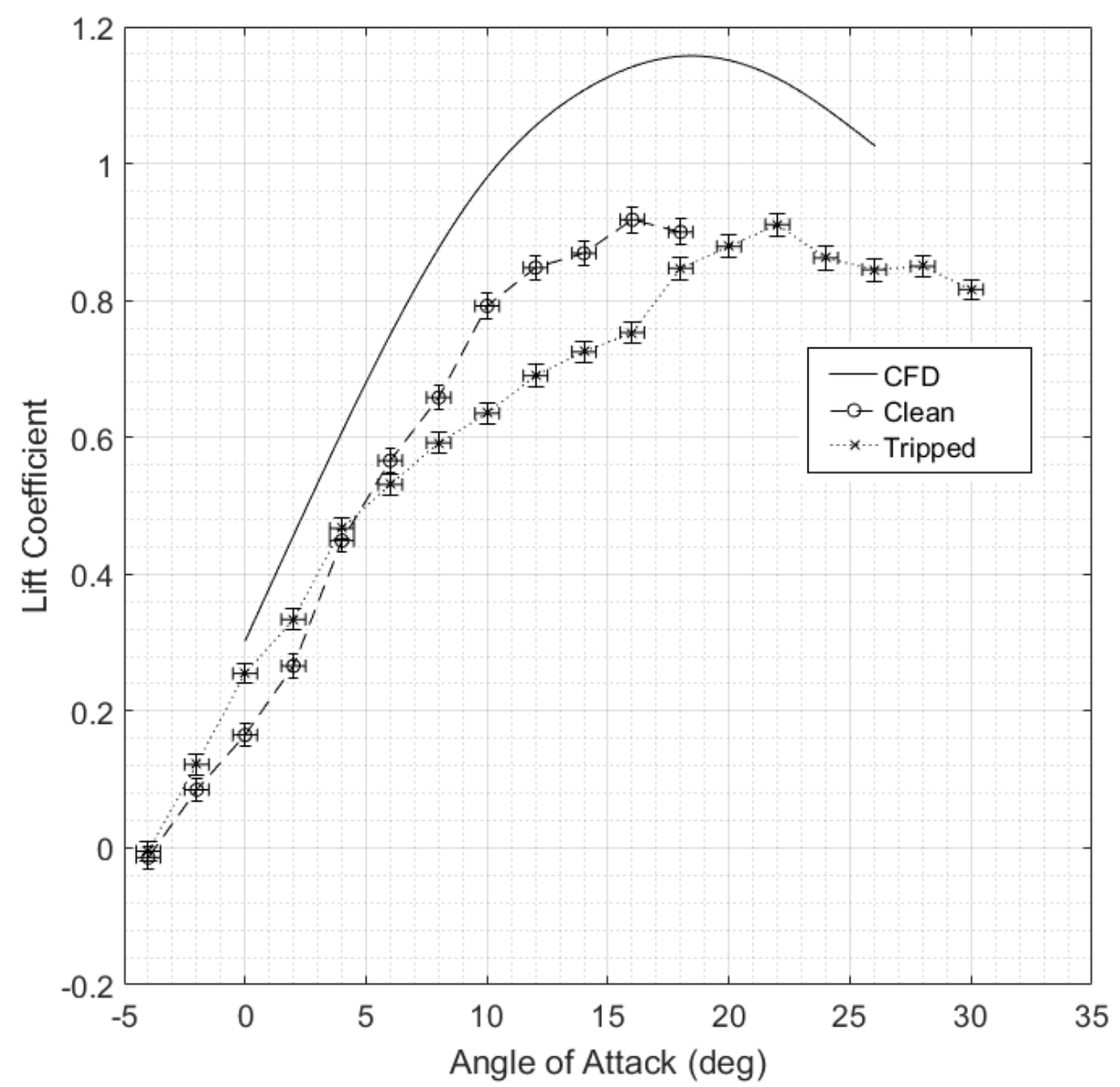

Figure 4.5: Wing-only test compared to the CFD results $-c_{L}$ vs. $\alpha$.

The simulation was run for angles of attack ranging from $0^{\circ}$ to $26^{\circ}$. The lift curve slope, $c_{L_{\alpha}}$, for the CFD result is greater than the slope for both experimental results as seen in Table 4.1. Furthermore, all results show that non-zero lift is generated at zero angle of attack. Through the experimental results, it can be observed that zero lift is produced at an angle of attack of approximately $-4^{\circ}$. By extrapolating from the CFD simulation for negative angles of attack values within the linear region, a $-4.5^{\circ}$ zero lift angle of attack is obtained. A maximum lift coefficient of 1.16 is also observed in the simulation; this is approximately $23 \%$ greater than the maximum 
lift coefficient observed during experimentation. Due to the simulation running at greater Reynolds number, a greater maximum lift coefficient is expected $[10,20,43$.

Table 4.1: Computational and experimental lift curve slope results

\begin{tabular}{|c|c|c|}
\cline { 2 - 3 } \multicolumn{1}{c|}{} & $c_{L_{\alpha}}\left(\mathrm{rad}^{-1}\right)$ & Percentage Difference \\
\hline CFD Result & 3.96 & \\
\hline Clean Configuration & 3.37 & $16 \%$ \\
\hline Tripped Configuration & 3.11 & $24 \%$ \\
\hline
\end{tabular}

Ahmed also compared the CFD result for $c_{L_{\alpha}}$ to theoretical result for a finite wing obtained from thin airfoil theory and by assuming the theoretical maximum two-dimensional lift slope of $2 \pi[10,27]$. This theoretical value is determined to be $3.38 \mathrm{rad}^{-1}$ 27]. This result is similar to the experimental result obtained for the clean configuration case. It should be noted that an Oswald efficiency factor of 0.75 was assumed when developing this theoretical result; this value will likely be lower due to the wing's moderate aspect ratio [44]. Moreover, the use of the maximum two-dimensional slope is an idealized case. Therefore, the results for $c_{L_{\alpha}}$ would likely see further reduction.

\section{Drag Coefficient}

An additional experiment was undertaken with only the sphere that interfaces with the wing and its supporting rods in the wind tunnel. The effect of drag cause by the sphere and connecting rods was subtracted from the total measured drag. Figure 4.6 illustrates the measured drag coefficient for both clean and tripped configurations. A significant increase in drag is observed for the tripped wing due to the added 
grit. Within the linear region of drag increase $\left(6^{\circ}\right.$ to $\left.18^{\circ}\right)$, the average increase in drag coefficient is approximately 0.087 . The tripped configuration also experiences a notable increase in drag at an angle of attack greater than $22^{\circ}$. When comparing the lift performance (Figure 4.4) to the drag at this angle of attack, it can be observed that the onset of stall occurs after this point.

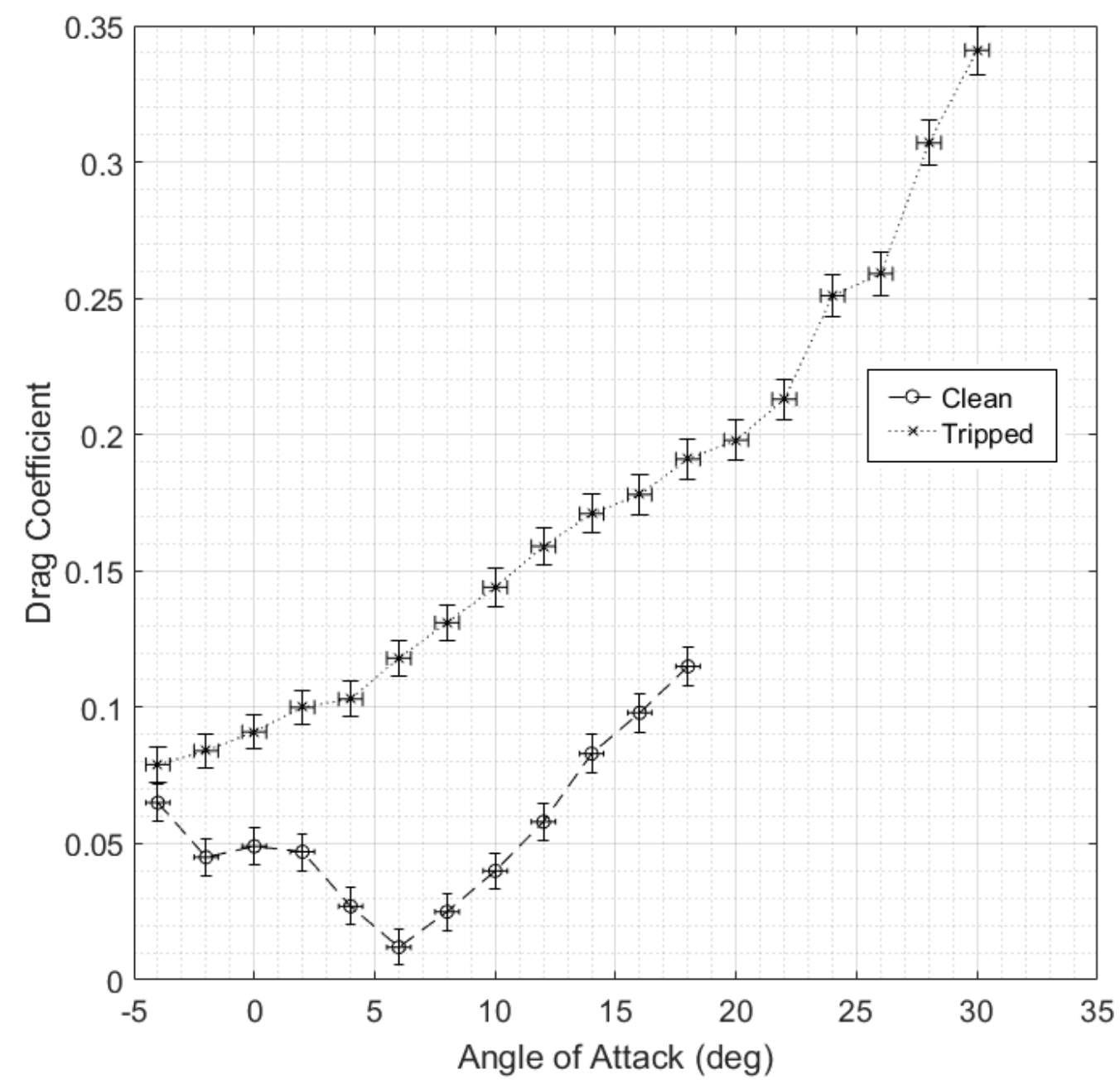

Figure 4.6: Wing-only test $-c_{D}$ vs. $\alpha$.

The overall change in the drag coefficient of the wing is relatively small over the range of angles of attack; this is an expected result due to the aerodynamically 
streamlined nature of the wing. A maximum drag coefficient increase of approximately 0.1 and 0.25 is observed for the clean and tripped wing respectively. Cook experimentally measured drag characteristics of a hang glider wing and observed minimal changes in the drag coefficient [8]. This becomes more apparent as speed increases. Cook concluded that this aerodynamic parameter is a pilot parameter in hang glider analysis [8]. In the case of ultra-light aircraft, it can be considered a pilot and carriage parameter.

From Figure 4.6, the small changes in $c_{D}$ are not evident. When these experimental results are shown in conjunction with the CFD results, the magnitude of the change in the drag coefficient becomes more apparent. The CFD results illustrate a quasi-linear increase in drag coefficient from $6^{\circ}$ to $18^{\circ}$ with a slope that is an order of magnitude greater than the slope for the experimental results. When comparing a two-dimensional wing to a finite wing, factors such as wing sweep, taper, span, and dihedral will all have an impact on the overall induced drag. However, these factors will not have as significant of an impact on the drag coefficient as those shown in the CFD simulation [45]. Furthermore, a negative drag value is observed for a zero degree angle of attack in the simulation; this implies that there is a net resultant force in the parallel and opposite direction of the oncoming flow. This result is distinctly erroneous.

From the lift and drag comparisons illustrated in Figures 4.5 and 4.6 , it is clear that the CFD simulation is not valid and must be revisited. The drag coefficient should not see such significant increase as angle of attack increases. Numerous experiments were conducted on hang glider wings, delta wings, and airfoils and no $16,41,45,46$ and such large drag coefficients were not observed. 


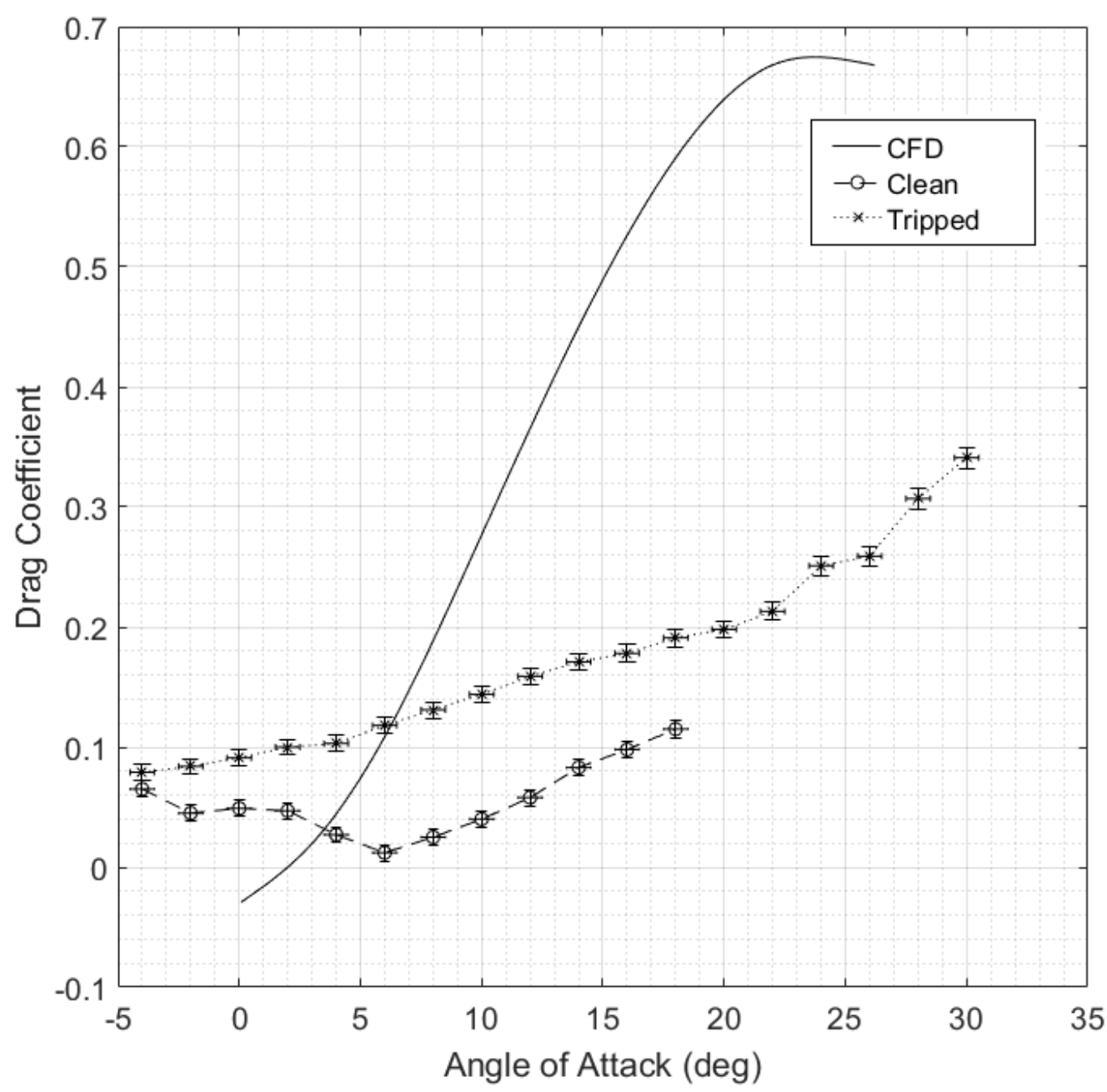

Figure 4.7: Wing-only test compared to the CFD results $-c_{D}$ vs. $\alpha$.

The fuselage and pilot are the source of the majority of the drag coefficient and therefore drag characteristics will be analyzed in more detail in the next section when the fuselage is included in the experimental investigation. 


\section{Pitching Moment Coefficient}

With respect to the pitching moment of the wing, no CFD analysis was undertaken. Therefore, the comparisons that are made in Figure 4.8 are exclusively between the clean and tripped wing configuration wind-tunnel testing. It can be seen that the wing possesses a positive pitch stiffness $\left(c_{M_{\alpha}}<0\right)$ which meets the longitudinal stability criteria. Therefore, it has a tendency to return to its trimmed position regardless of its angle of attack. The results for the pitch stiffness slope, zero angle of attack pitching moment coefficient, and the trimmed angle of attack are shown in Table 4.2,

Table 4.2: Pitch stiffness slope, zero angle of attack pitching moment, and trim angle of attack for wing-only experiments.

\begin{tabular}{|c|c|c|}
\cline { 2 - 3 } \multicolumn{1}{c|}{} & Clean & Tripped \\
\hline$c_{M_{\alpha}}\left(\mathrm{rad}^{-1}\right)$ & -9.17 & -8.38 \\
\hline$c_{M_{0}}$ & 0.67 & 0.34 \\
\hline$\alpha_{\text {trim }}(\mathrm{deg})$ & 8.8 & 10.6 \\
\hline
\end{tabular}




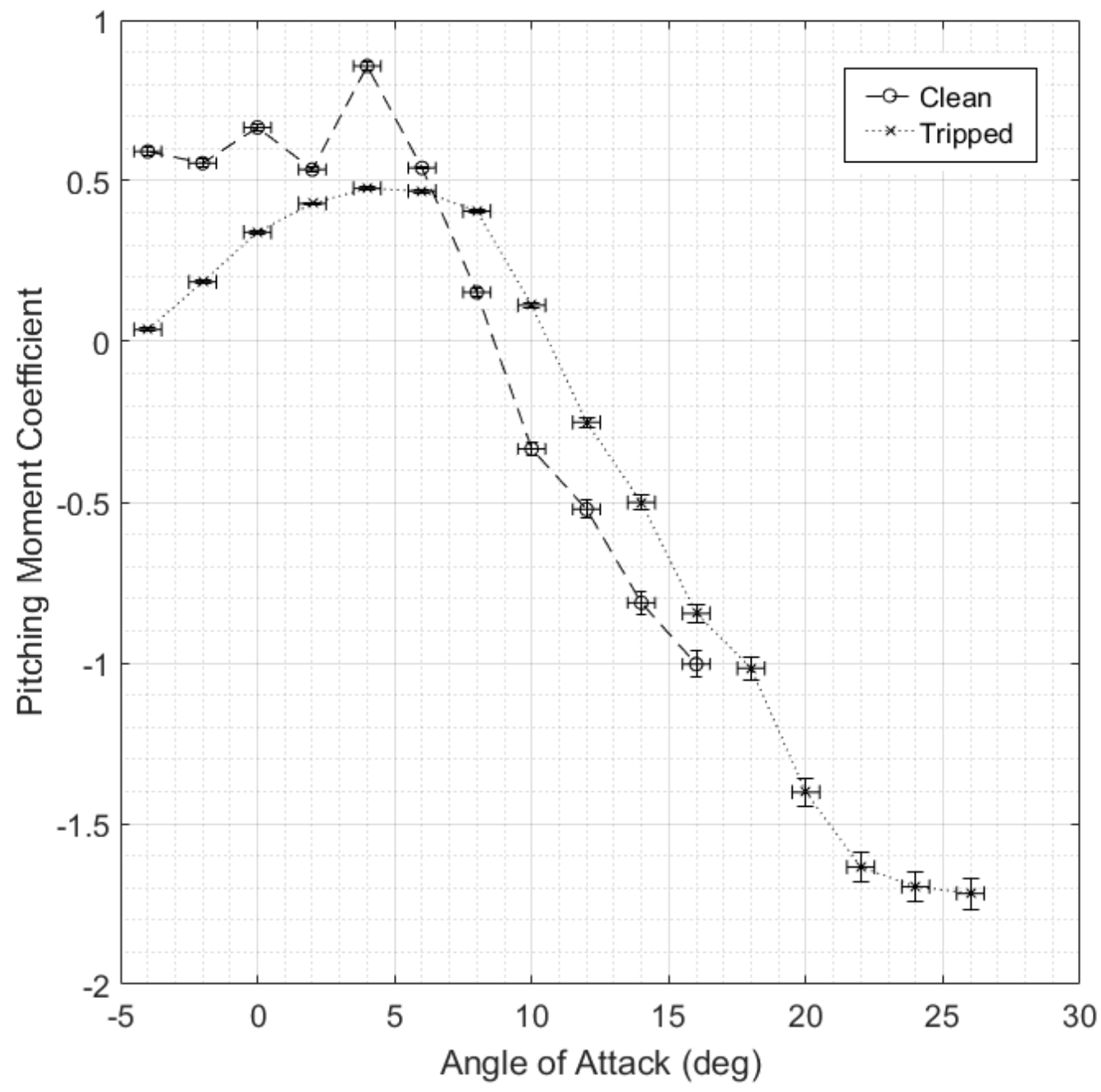

Figure 4.8: Wing-only test $-c_{M}$ vs. $\alpha$.

The results obtained for the trim angle of attack for this the wing are in line with the typical results obtained from full scale experimentation [8,41]. Previous experimental results also suggest that the effects of the pitching moment with respect to the angle of attack are expected to be non-linear [8, 16, 41]. From the results obtained from wind tunnel testing, these non-linearities are apparent for lower angles of attack $\left(\alpha<4^{\circ}\right)$. The parabolic trend at lower angles of attack have been 
observed in both experimentation and through mathematical modelling [8, 16, 41, 47. A key difference that is observed in the results in Figure 4.8 is the linear trend that occurs as the angle of attack increases. The pitching moment coefficient becomes highly negative and the magnitude of the $c_{M}$ values become notably large. The magnitudes of these pitching moment coefficients are significant compared to existing experimental results $8,16,41$. These existing results do not show hang glider wings attain pitching moment coefficients greater than -0.5 whereas the experimental results in Figure 4.8 show pitching moment coefficients of approximately -1.75 . The rigid nature of the scale model wing likely has an impact on these results. Wing twist will cause less lift to be generated near the wing tip. Given that a portion of the wing is located behind the centre of gravity, the magnitude of the pitch stiffness will decrease if the wing is flexible.

It should also be noted that the full scale experiments undertaken by Cook and Kilkenny were conducted over 30 years ago on single surface hang glider wings at relatively lower speeds $8,16,41]$. The flexibility of these wings, particularly at lower speeds, will have a significant impact on the pitching behaviour of the aircraft 41 . There have been a number of single and double surface wings that have been developed specifically for ultra-light trikes in recent years [6].

\subsection{Ultra-light Model}

\subsubsection{Test Case 1}

The results from test case 1 provide information regarding the aircraft's lift curve, drag polar, pitching moment and an indication of its degree of longitudinal static stability. 


\section{Lift Coefficient}

The experimental results of the aircraft's lift curve are illustrated in Figure 4.9 From this curve, it is apparent that the addition of the trip strip will increase the maximum lift generated. This was not the case for the wing-only configuration and it is not an expected results compared to previous work [32, 48]. Although the trip strip delayed stall when testing the wing in isolation, it came at a penalty in lift generation. With regards to the ultra-light model, the test without the trip strip begins to show the onset of stall at an angle of attack of approximately $25^{\circ}$ whereas the trip strip will delay stall passed $30^{\circ}$ angle of attack. Similar to the wing-only configuration, it is likely that the boundary layer will separate from the surface leading to a loss in lift without the addition of a trip strip. The stall characteristics of the ultra-light model were not obtained due to the constraints of the test section.

The linear region of the lift curve at low angles of attack for both tripped and clean configuration illustrate that the slope remains similar for both configurations. At an angle of approximately $10^{\circ}$, the magnitude of the slope of the lift curve for the clean configuration begins to drop whereas the tripped wing only experiences small signs of reduction in $c_{L_{\alpha}}$. 


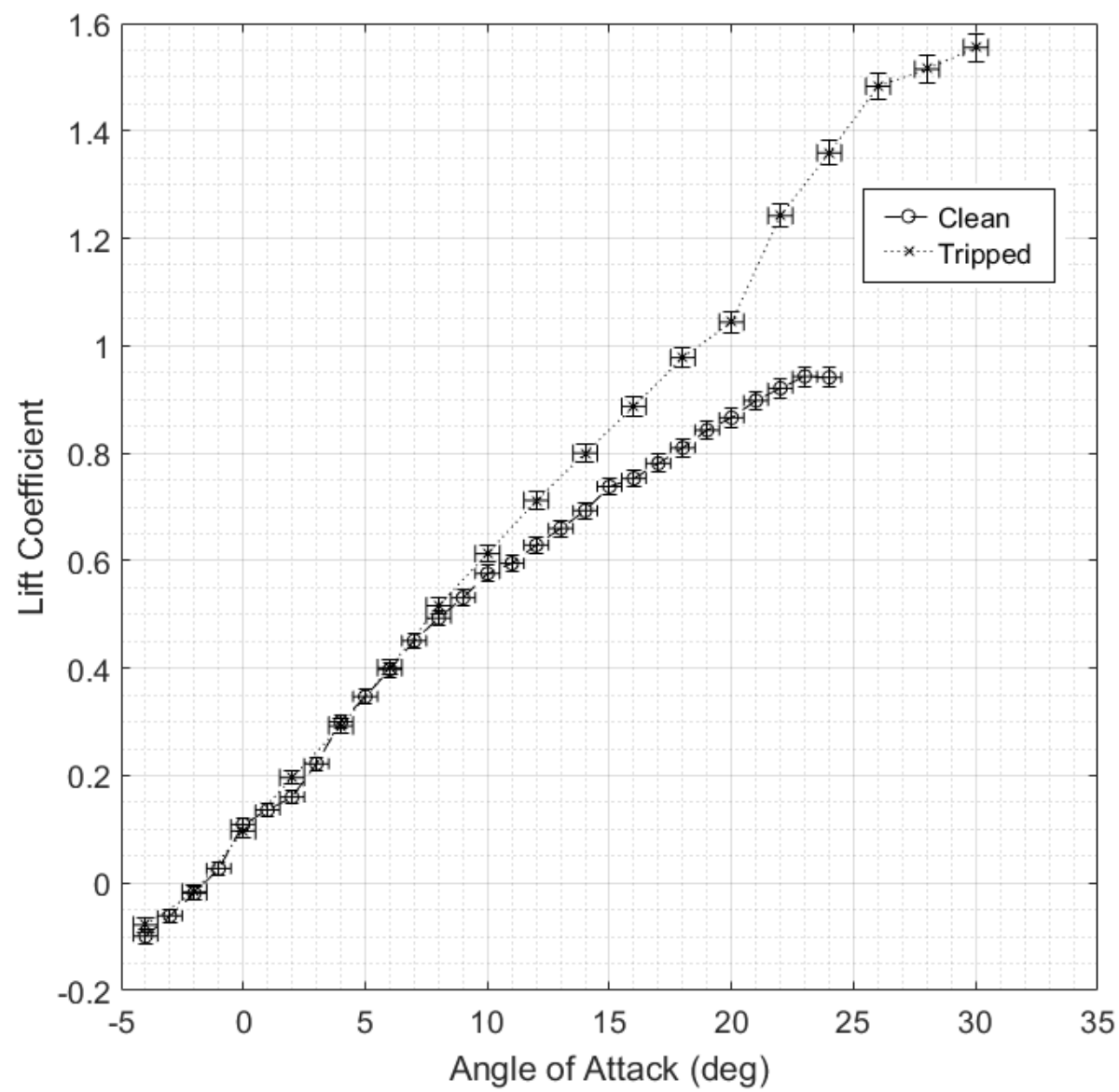

Figure 4.9: Ultra-light model test $-c_{L}$ vs. $\alpha$.

A comparison of the lift characteristics between the wing-only and ultra-light model are made in Figure 4.10 in order to quantify the aerodynamic loading effects due to the modifications to the wing's trailing edge at its root. These comparisons are made in the clean configuration for both models in order to limit the number of variables that have been changed. Although the ultra-light model includes the fuselage, it is assumed that the fuselage is a negligible lifting surface and that the majority of the lift is generated by the wing. To accurately quantify the lifting effect of the fuselage, a wind-tunnel test should be run for the fuselage in isolation. Considering that the fuselage is still in its preliminary design phase, it is 
recommended to quantify the aerodynamic performance of the fuselage in order to improve on its design.

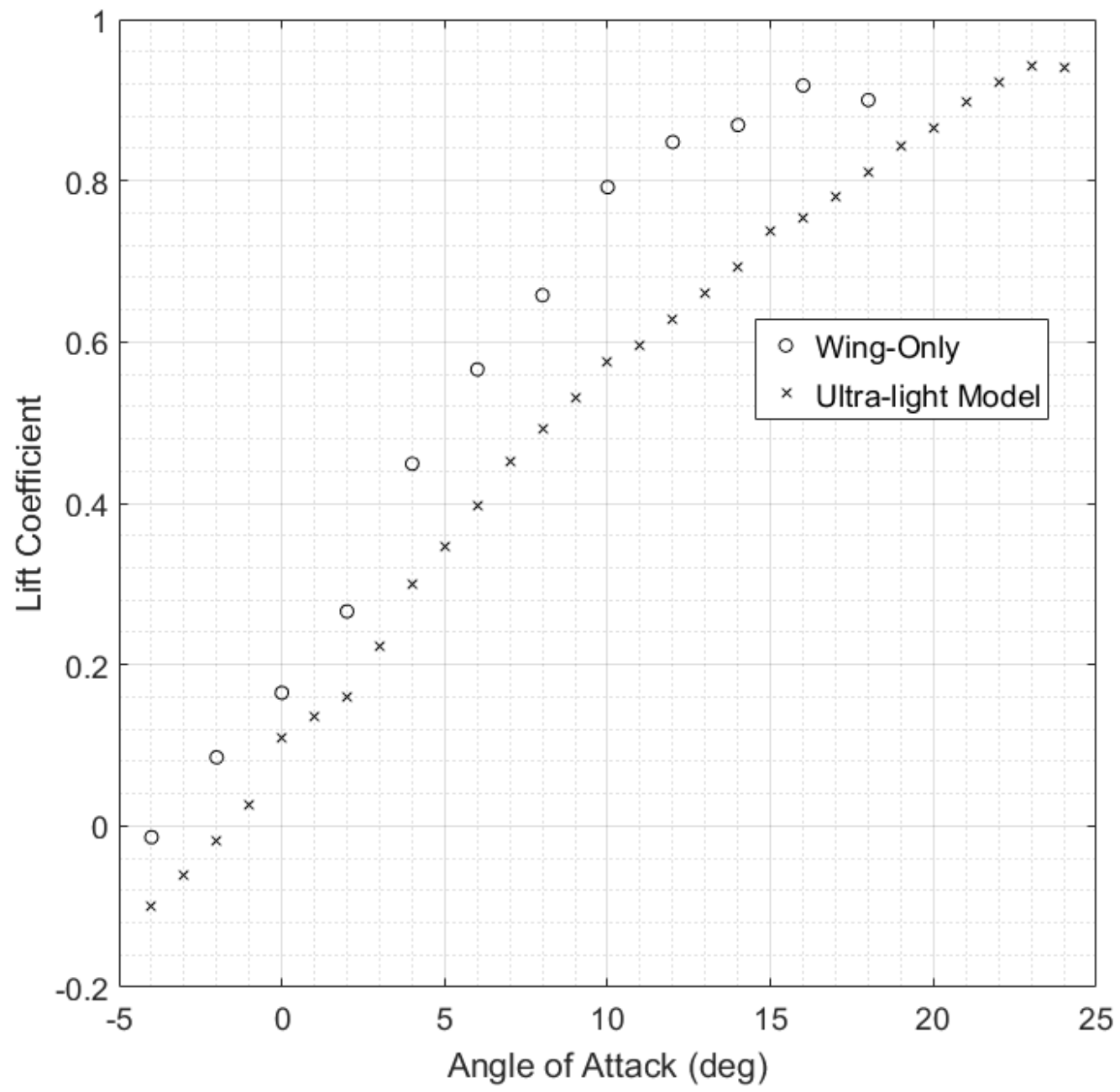

Figure 4.10: Wing-only and ultra-light model $-c_{L}$ vs. $\alpha$.

When comparing the lift coefficient results obtained for both the wing-only and ultra-light model, there are certain distinctions that become apparent due to the modification of the wing. The lift-curve slope shows an $11 \%$ decrease for the ultra-light model as well as an overall reduction in its lift coefficient for equivalent 
angles of attack within the linear region $\left(\alpha<10^{\circ}\right)$. The ultra-light model experiences a delayed stall along with an increase maximum lift coefficient. The onset of stall for the ultra-light model is observed at an angle of attack of $23^{\circ}$. Furthermore, an increase of $4 \%$ is seen in the maximum lift coefficient. From these results, it is important to note that small changes to the physical geometry of the wing could produce significant changes to the aircraft's performance.

\section{Drag Coefficient}

The drag polar for the aircraft is illustrated Figure 4.11. The total drag acting on the aircraft is divided into parasitic drag, $c_{D_{0}}$, and lift-induced drag. Assuming the aircraft has a typical parabolic drag polar where the lift-induced drag is a function of $c_{L}^{2}$, the drag expression is given by [49]:

$$
c_{D}=c_{D_{0}}+\frac{c_{L}^{2}}{\pi A R e}
$$

The parasitic drag is the net drag when the aircraft is at its zero-lift angle of attack (i.e. $c_{L}=0$ ). From Figure 4.11, the parabolic shape is observed at small $c_{L}$ and $c_{D}$ values. However, as the lift coefficient increases, the drag appears to increase linearly rather than parabolically. There are some explanations as to why the drag polar does not conform to the typical parabolic shape. Equation 4.1 assumes that the parasitic drag remains constant. However, due to the bluff shape of the fuselage, as the angle of attack increases, more surface area of the fuselage is exposed to the oncoming flow. This, in turn, will cause the parasitic drag to increase. Furthermore,

for hang glider wings, Kroo found that the drag relation shown in Equation 4.1 is unsuitable [15]. The parasitic drag for both the fuselage and wing is related to the friction drag and pressure drag due to separation [38,50]. As the angle of 
attack increases, separation is like to occur on the fuselage causing an increase in drag. Furthermore, previous experimental results show that the friction drag has a significant dependency on the lift force [15, 48,51]. Given that the Reynolds number is the ratio of inertial to viscous forces, the relatively lower Reynolds number will also contribute to a drag increase [51]. Other factors such as the surface roughness on the wing also potentially affect the shape of the drag polar 48].

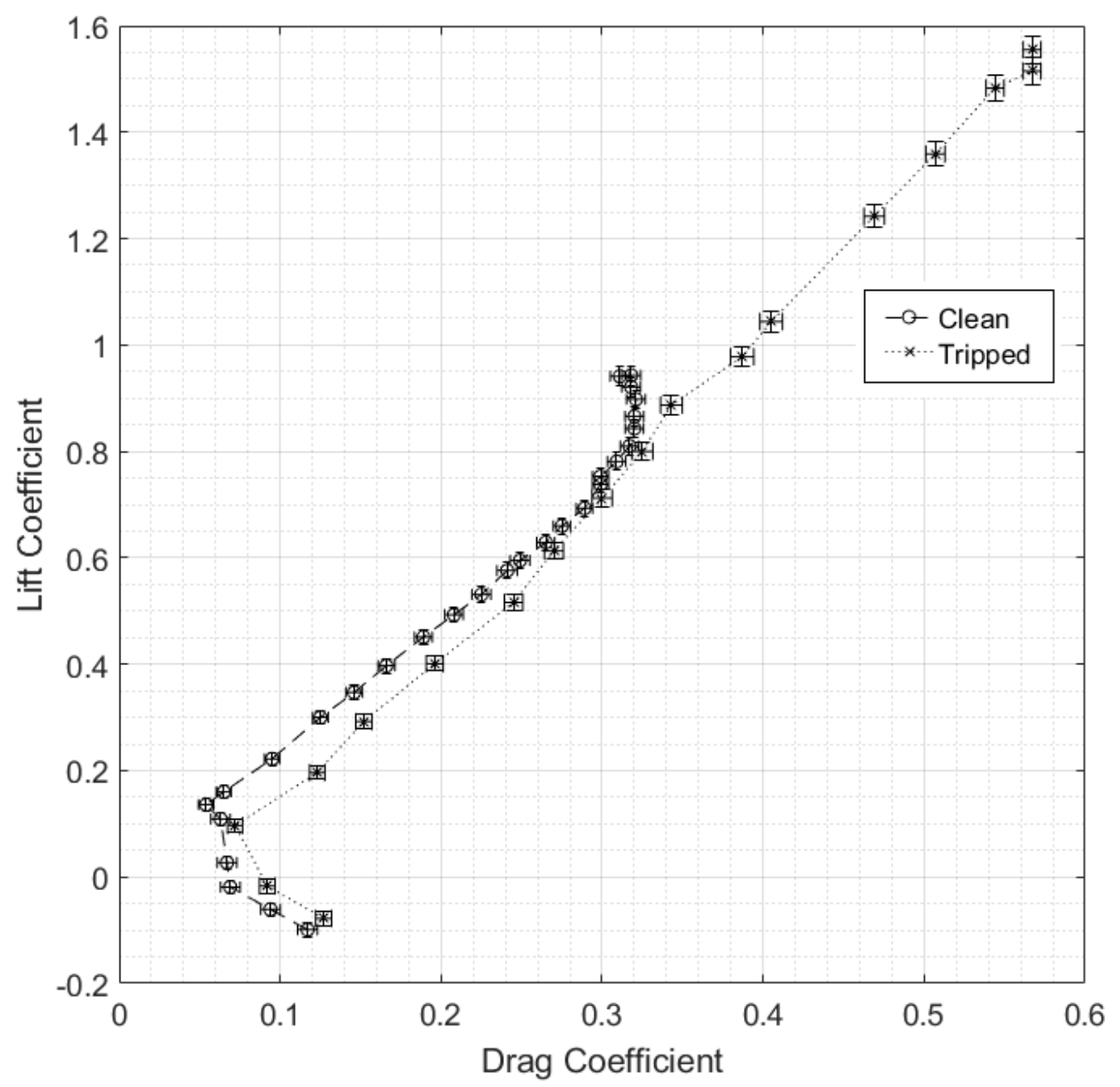

Figure 4.11: Drag polar of the ultra-light model test $-c_{L}$ vs. $c_{D}$ 
Figure 4.12 compares the drag polar of the wing-only test to the wing and fuselage test. From these results, it can be seen that both wing-only configurations (clean and tripped) exhibit typical drag polar behaviour. Therefore, it is clear that the interaction between the wing and fuselage will result in an increase in drag that cannot be only defined by Equation 4.1 .

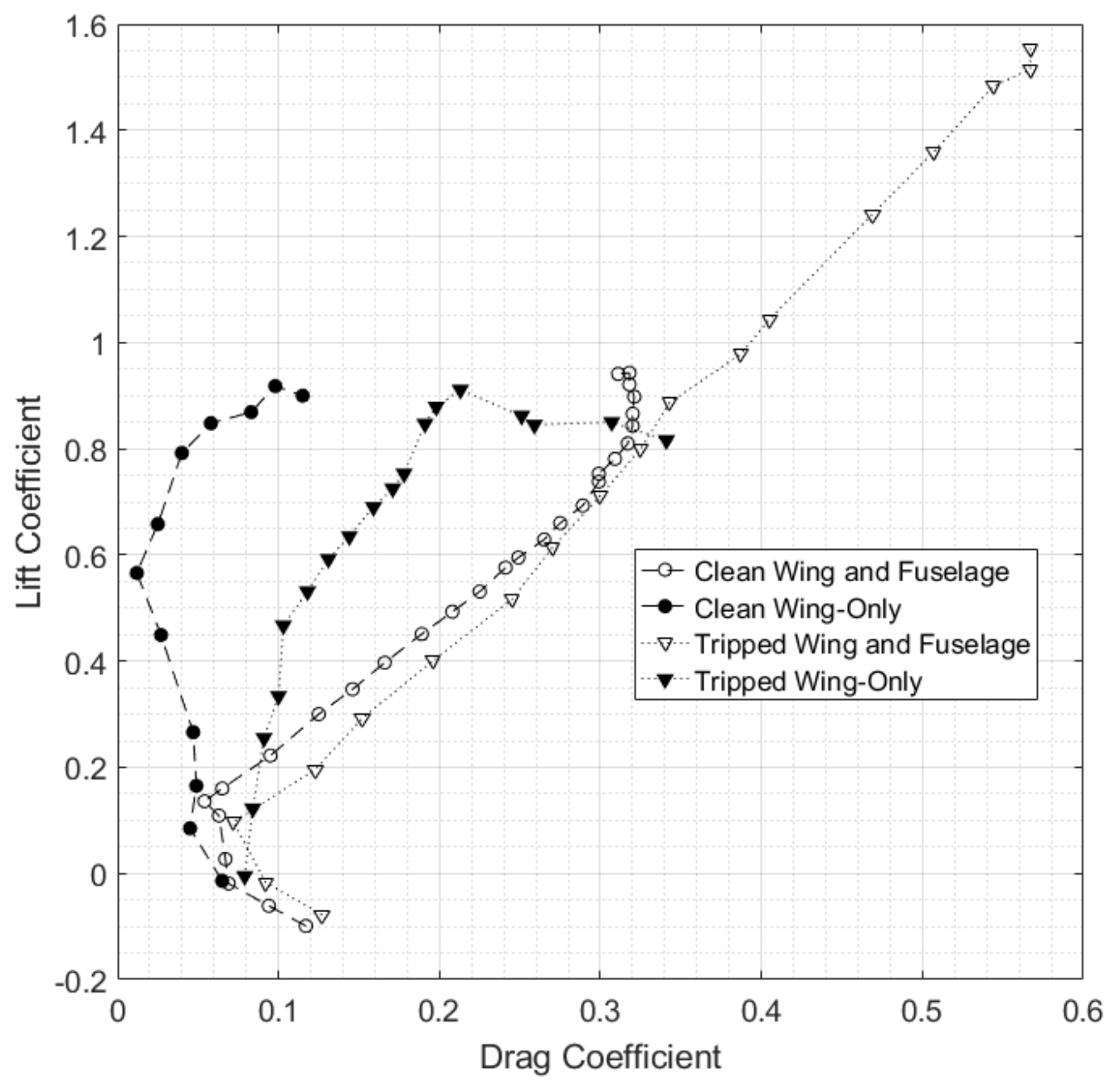

Figure 4.12: Drag Polar of the ultra-light model and ultra-light wing $-c_{L}$ vs. $c_{D}$

Raymer developed an expression for the parasitic drag in subsonic conditions as a function of all components of the aircraft, their interference factor (points of 
intersection between two components), $Q$, the skin friction drag $c_{f}$, pressure drag caused by separation (also referred to as form factor, $F F$ ) [38]:

$$
c_{D_{0}}=\frac{\sum_{\mathrm{i}=1}^{n}\left(c_{f} F F Q S_{\mathrm{wet}}\right)_{\mathrm{i}}}{S}+c_{D_{\mathrm{misc}}}+c_{D_{\mathrm{L \& P}}}
$$

where $n$ is the number of components, $S_{\text {wet }}$ is the wetted area of the aircraft, $c_{D_{\text {misc }}}$ is miscellaneous drag due to factors such as unswept aft fuselage, and $c_{D_{\mathrm{L} \& \mathrm{P}}}$ is the drag due to leakages and protuberances.

The parasitic drag of the clean configuration model is seen to be smaller than that of the tripped model. This is an expected result due increased drag caused by the addition of surface roughness on the tripped model.

The performance characteristics of the aircraft are important when undertaking the design of the fuselage. It allows for the ability to change the design in order to improve the overall lift-to-drag ratio of the aircraft.

\section{Pitching Moment Coefficient}

The pitching moment coefficients for the ultra-light model are similar to those seen in the wing-only experiments. From Table 4.3, with regards to the pitch stiffness, $c_{M_{\alpha}}$, the values are comparable to those of the wing-only configuration. From this, it can be inferred that the magnitude of the pitch stiffness is dominated by the wing rather than the fuselage. Moreover, the trip strip also causes a reduction in the pitching moment coefficient. The most notable change is the zero angle of attack pitching moment coefficient and the trim angle of attack. The addition of a fuselage on the model will place the aircraft's centre of gravity at a location between the fuselage and wing as illustrated in Cook and Spottiswoode's hang glider model (Fig 
2.10 [16]. Therefore, an additional nose-down pitching moment about the aircraft's centre of gravity will be induced due to the considerable drag caused by the fuselage.

Table 4.3: Pitch stiffness slope, zero angle of attack pitching moment, and trim angle of attack: ultra-light model.

\begin{tabular}{|c|c|c|}
\cline { 2 - 3 } \multicolumn{1}{c|}{} & Clean & Tripped \\
\hline$c_{M_{\alpha}}\left(\mathrm{rad}^{-1}\right)$ & -9.26 & -8.33 \\
\hline$c_{M_{0}}$ & 0.183 & 0.206 \\
\hline$\alpha_{\text {trim }}(\mathrm{deg})$ & 1.6 & 1.8 \\
\hline
\end{tabular}

From the results shown in Figure 4.13 , the linearity as angle of attack increase is apparent. As the angle of attack increases, the pitching moment coefficient increases in magnitude rapidly. The load cell measuring these pitching moments attained its maximum capacity at an angle of attack of approximately $11^{\circ}$. When comparing these results to full scale experimental results on hang glider wings, the typical $c_{M}$ vs. $\alpha$ graphs will see high non-linearities due to deformable nature of the wing [8, 15, 52. The rigid wing exhibits more linear pitching moment characteristics at greater angles of attack as compared to flexible wings 15, 16, 53. 


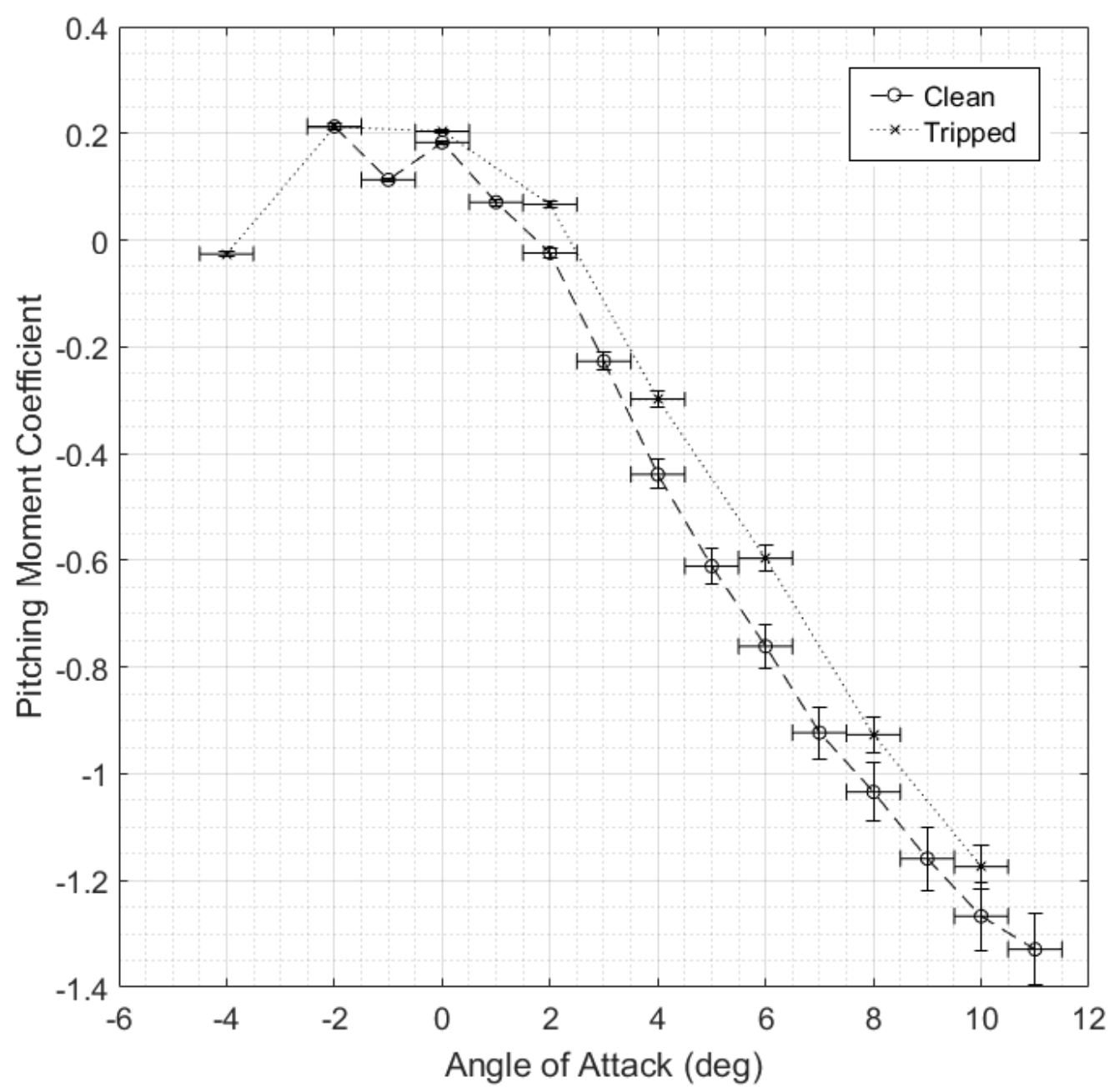

Figure 4.13: Ultra-light model test $-c_{M}$ vs. $\alpha$.

It should be noted that the aforementioned experiments on full scale hang glider wings were undertaken between 1970 and 1990. Modern wings are designed with more pitching moment stability 15 . 


\subsubsection{Test Case 2}

Test case 2 evaluated the lateral static stability in yaw for the ultra-light model. A test was only undertaken on the model with the trip configuration. The side force, $c_{Y}$, that acts along the aircraft's lateral axes and the yaw moment, $c_{N}$, that acts about the aircraft's normal axes are influenced by the aircraft's sideslip angle and the resulting drag. Due to the ultra-light model not having a vertical tail surface, the component of the drag vector acting along the lateral axis of the aircraft will generate the side force. The lift vector does not impact the yaw stability as it acts along the axes of rotation. Therefore, the trip strip's affect on the yaw stability is purely due to the additional drag that it generates. Moreover, the wing's contribution to the overall side force and yaw moment is negligible with respect to the fuselage [11].

\section{Yaw Moment Coefficient}

The yaw moment was measured with respect to the aircraft's sideslip angle in order to determine wether the aircraft is statically stable in yaw (also referred to as weathercock stability $[12$ ). The results of the experiment are illustrated in Figure 4.14. 


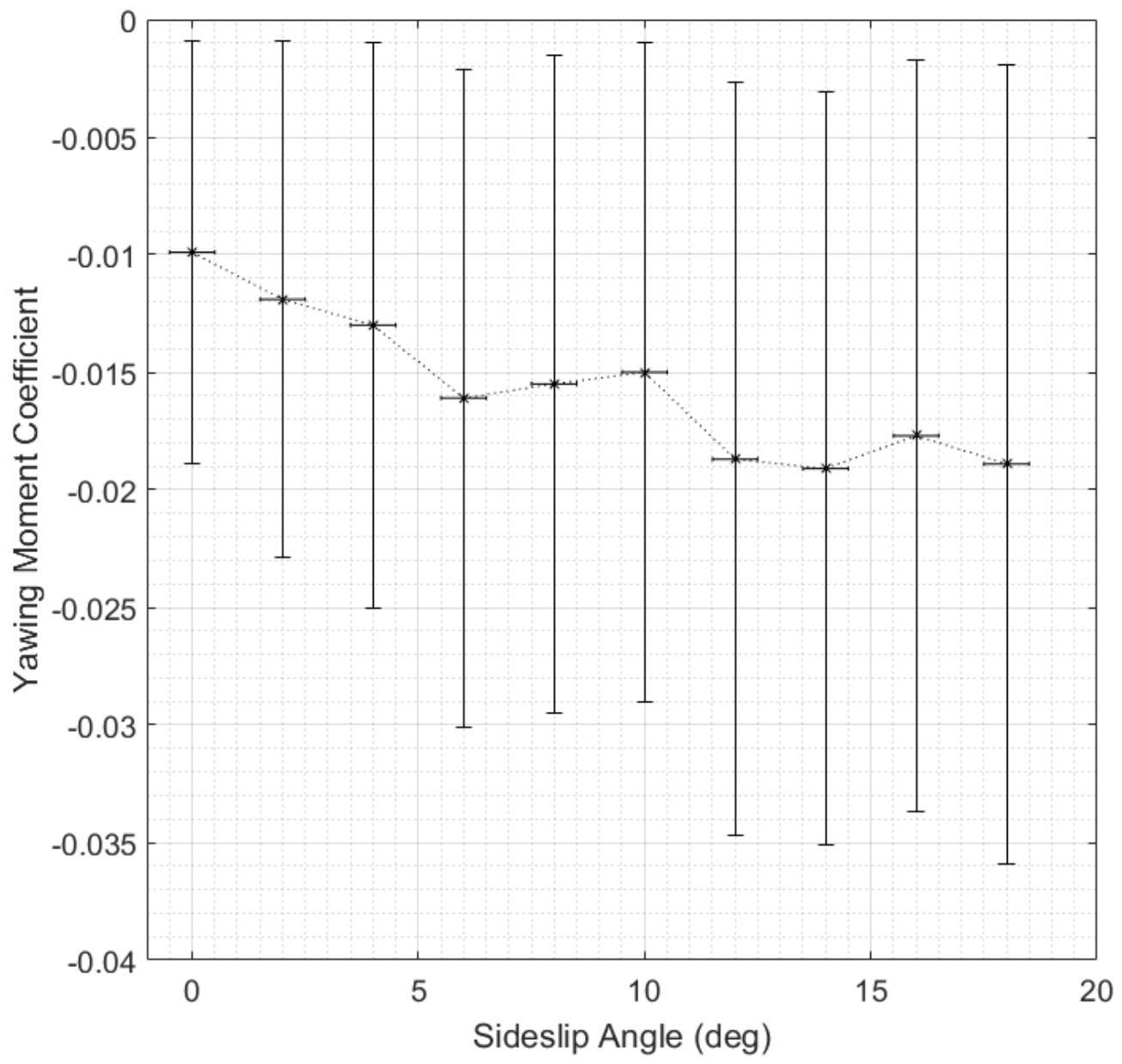

Figure 4.14: Ultra-light model test $-c_{N}$ vs. $\beta$.

As per the definition given in Equation 2.1, $c_{N_{\beta}}$ must be greater than 0 to satisfy weathercock stability. From Figure 4.14, it can be inferred that this condition is not satisfied. It is likely that the aircraft is not stable in yaw due to the lack of a vertical tail fin. A tail place behind the aircraft's centre of gravity will result in a lateral force that attempts to yaw the aircraft toward the relative flow direction [11, 12]. It is important to note that the magnitude of the yaw moment coefficient is small. The same load cell that measures the change in pitching moment on the order of $3.5 \mathrm{~N} \cdot \mathrm{m}$ was used to measure the yaw moment. The overall recorded change in yaw moment 
in this experiment is approximately $0.05 \mathrm{~N} \cdot \mathrm{m}$ (approximately $1.4 \%$ of maximum load cell capacity) and therefore accuracy issues are evident as seen by the large error bars.

Although the experiment lacks accuracy, comparing the yawing moment due to sideslip to existing experimental results [15,54, it can be seen that $c_{N_{\beta}}$, although negative, is relatively small and therefore the instability effects can corrected by pilot inputs or additional fins on the carriage [6].

Another important observation is that the resulting trendline does not cross the origin; this would naturally be the case in symmetric flight conditions due to the aerodynamic loads being equally distributed along both halves of the aircraft. The same issue is observed in the side force measurements and potential causes will be discussed at the end of the next section.

\section{Side Force Coefficient}

When observing the results for the side force loading with respect to the change in sideslip angle (Figure 4.15), the side force vector appears to change from the positive $\mathrm{y}$-direction to the negative $\mathrm{y}$-direction and a negative $c_{Y_{\beta}}$ slope is observed. Historical trends would indicate that negative slopes are expected for both hang gliders and conventional aircraft [15,54]. With regards to $c_{Y}$, when the aircraft is in a sideslip, the net resulting force on the aircraft in the lateral direction would only be the component of the drag vector along that axis; this would therefore lead to negative side forces (with respect to the body-axes convention) that will increase in magnitude as the area of the fuselage becomes more exposed to the oncoming flow. Thus, $c_{Y}$ should be negative for any positive sideslip angle and should be zero at $\beta=0$. The results are not indicative of this type of behavior. 


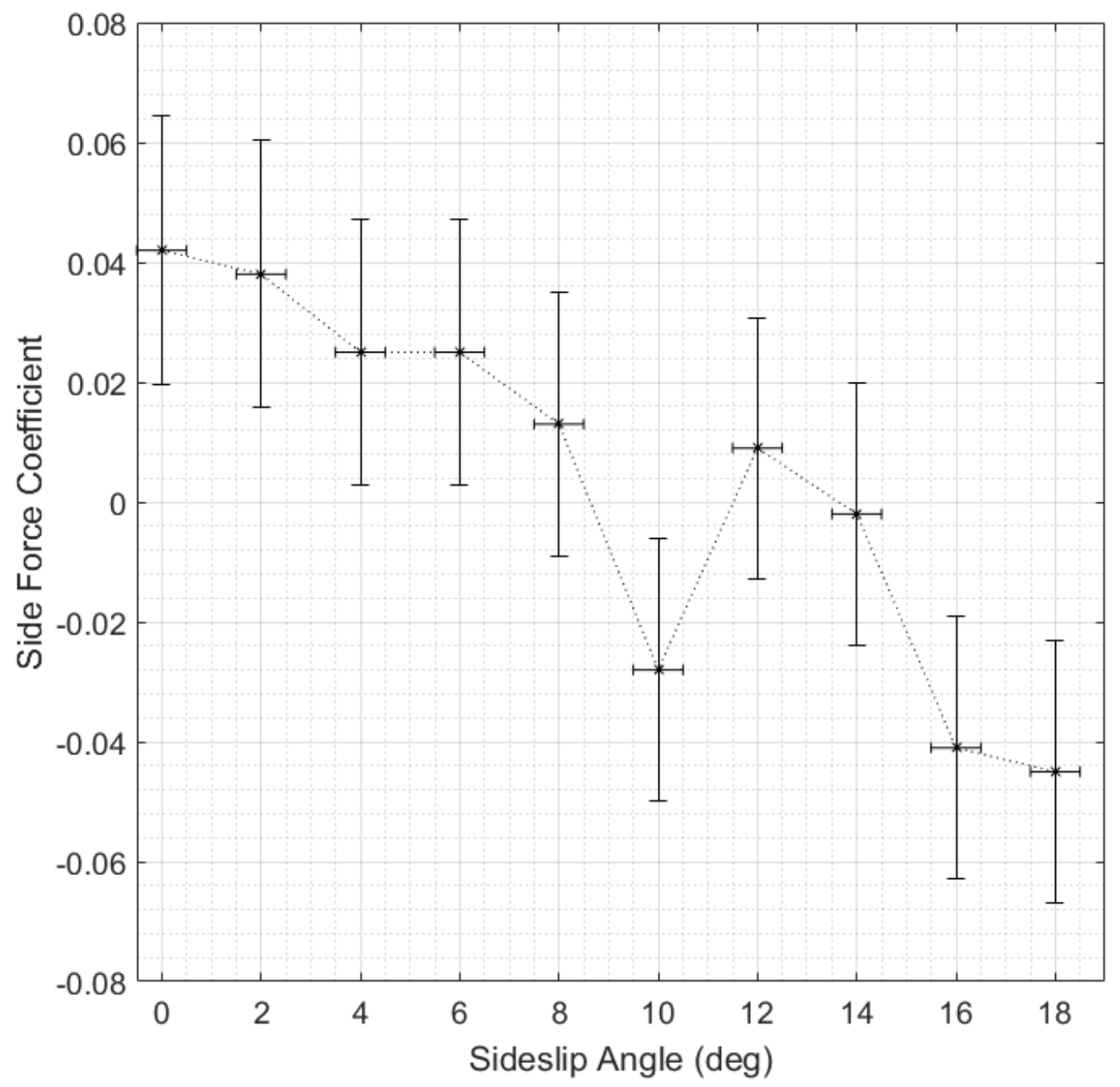

Figure 4.15: Ultra-light model test $-c_{Y}$ vs. $\beta$.

Similar to $c_{N}$, the side force coefficient has orders of magnitude that are significantly smaller than the drag and lift coefficients developed in test case 1 . The load cells that were used to measure these aerodynamic loads were also used to resolve $c_{Y}$ by transforming the measured loads from wind-axes to body-axes (See Appendix A for transformations between axes systems). Measured lift and drag forces were up to 2 orders of magnitude greater than the side force. The overall recorded change in side force was approximately $0.6 \mathrm{~N}$ ( or $60 \mathrm{~g}$ ). This implies that the slightest changes in 
the balance or drift in the voltage (on the order of 1/1000 V) could lead to spurious results. Therefore, the error bars are significant. From these types of tests, linear changes in the side force are to be expected but it is clear that the resolution is not adequate for accurate readings.

A notable issue that affected the results for both $c_{N}$ and $c_{Y}$ was the zeroing of the balance. The balance was zeroed prior to powering up the wind-tunnel. It is likely that mechanical stresses that were applied on the balance as the wind-tunnel velocity was increased to the required test conditions were responsible for a non-zero loading in the wind-axes normal direction as well as a non-zero yawing moment at $\beta=0$. If the recorded voltages at zero sideslip where to be set as the zeroed values, both $c_{N}$ and $c_{Y}$ would be zero. Thus, the results would appear to be more representative of actual yaw moments and side forces. These comparisons can be seen in Figures 4.16 and 4.17. From these corrections, it can be seen that both trends pass through the origin and all side force coefficients are now negative as would be expected. 


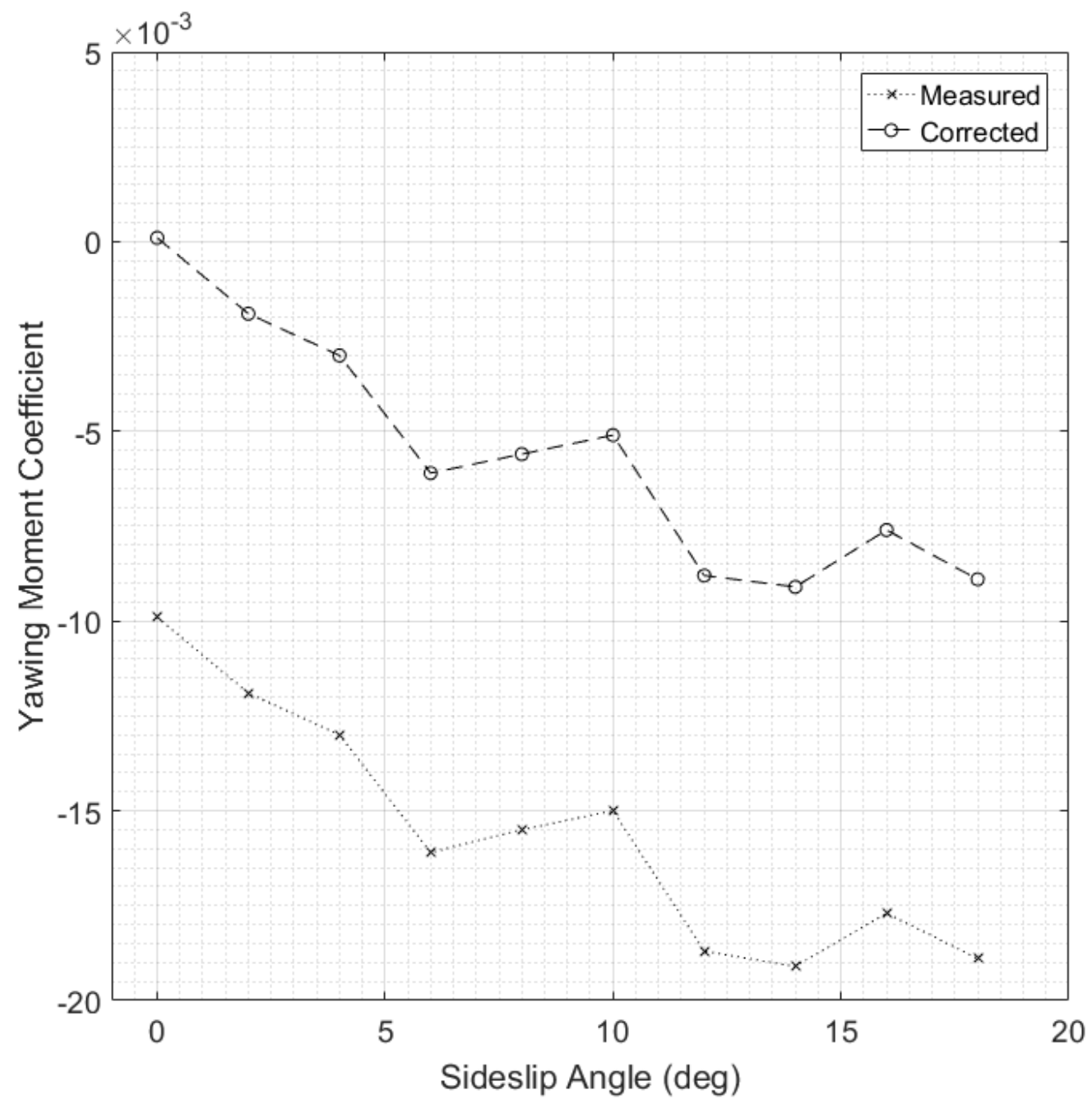

Figure 4.16: Ultra-light model test $-c_{N}$ vs. $\beta$ (corrected). 


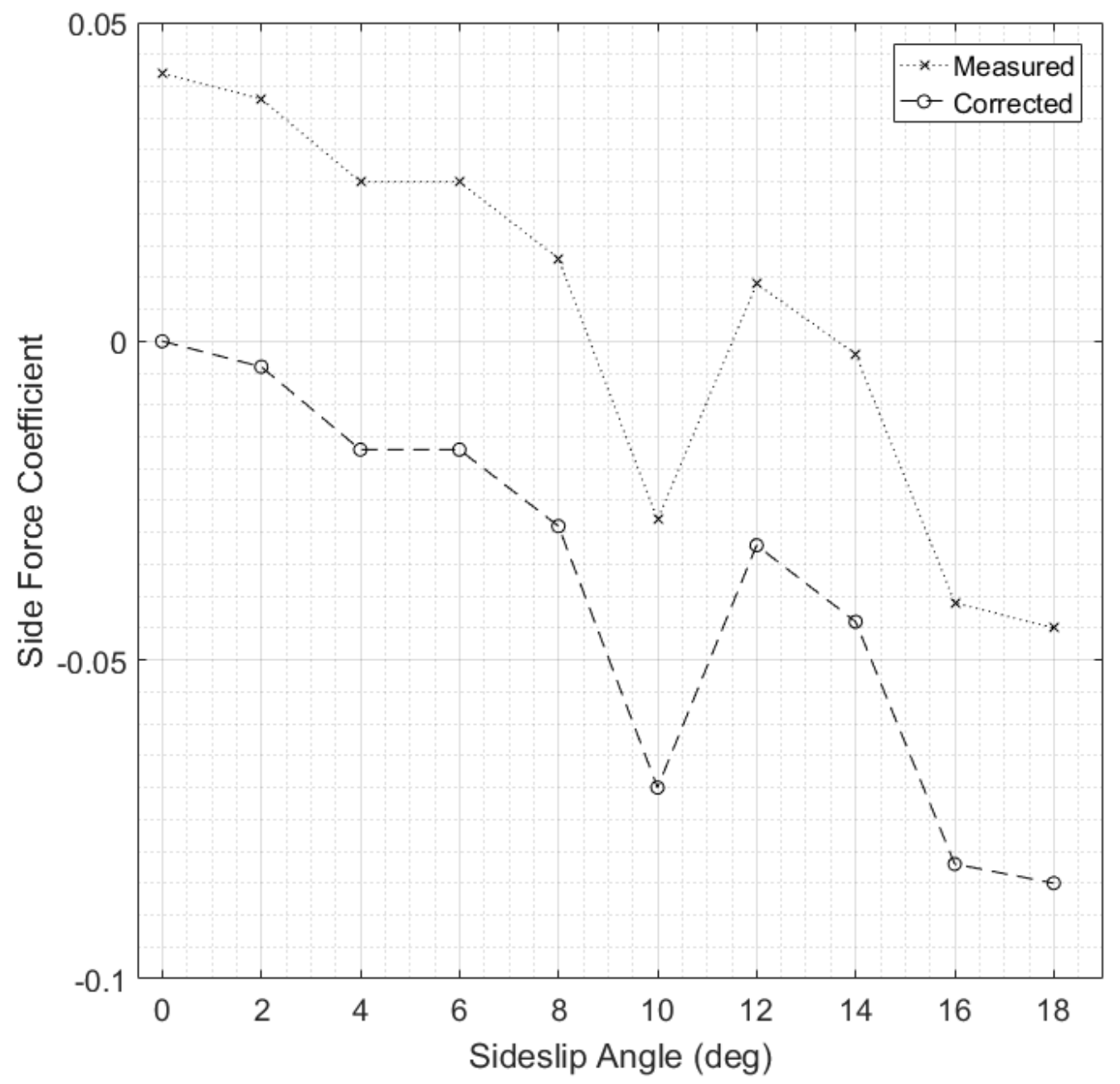

Figure 4.17: Ultra-light model test $-c_{Y}$ vs. $\beta$ (corrected).

As an alternative, the side force may also be resolved by taking the measured yaw moment about the centre of gravity and solving for the side force:

$$
N=Y \cdot d
$$

where $d$ is the distance from the fuselage's centre of gravity to the aircraft's centre of gravity. This assumption implies that the wing's effect on the side force is negligible and that the fuselage is the only factor responsible for this side force. 
In reality, this will not be the case as the wing will have some contribution to the overall side force. Due to the magnitude of the applied loads, this assumption allows the side force to be calculated in a simpler fashion by putting emphasis on the fuselage.

Equation 4.3 can be rewritten in terms of yaw and side force coefficients from the equations listed in Table 2.1.

$$
\frac{N}{\frac{1}{2} \rho V^{2} S b}=\frac{Y}{\frac{1}{2} \rho V^{2} S} \cdot \frac{d}{b}
$$

Expression 4.4 will then simplify to:

$$
c_{N}=c_{Y} \cdot \frac{d}{b}
$$

The results of obtained from solving Equation 4.5 for $c_{Y}$ given the measured $c_{N}$ values are shown in Figure 4.18. From these results, it it can be observed that the trend and magnitude of the calculated side force values are similar to measured values. The results will still lack accuracy and it is evident that there are inconsistencies as the trends are not linear. This is most notable at sideslip angles of $10^{\circ}, 16^{\circ}$, and $18^{\circ}$. Small increases in voltage on the order of $30 \mathrm{mV}$ were recorded by the flow-normal load cell. Due to the dominance of the flow-normal load cell when resolving the side force (cosine of small angles), any recorded changes will have a noticeable impact on the calculated side force. The spurious results seen at the aforementioned sideslip angles may be a result of stress relaxation in the external balance connection points as sideslip angle changes; it may also be caused by interactions between the support rods and the upper wall of the test section. It should be re-iterated that measurements taken are on the order of 10 grams and thus measurement errors are likely to occur. 


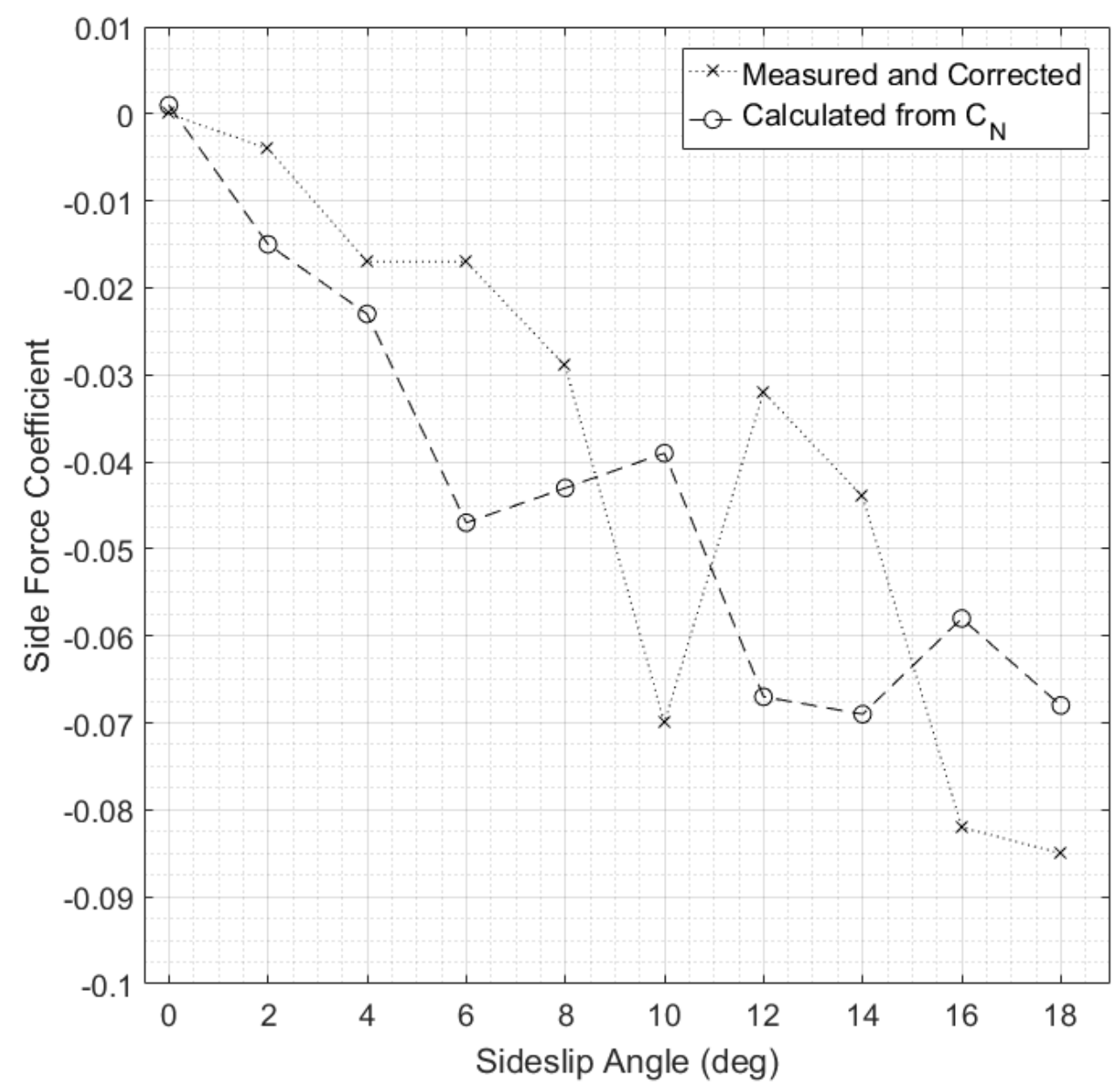

Figure 4.18: Ultra-light model test $-c_{Y}$ vs. $\beta$ (measured and calculated).

As mentioned above, the drag on the aircraft is the only force that has an impact on the side force; the drag is measured in the flow-parallel (or axial) direction. Therefore, flow-normal loads should be zero. For all sideslip angles except $10^{\circ}, 16^{\circ}$, and $18^{\circ}$, this holds true. Thus, another potential correction that can be made with regards to the side force is the removal of the loads measured by the flow-normal load cell as they should ultimately be zero. The results of this correction is shown in Figure 4.19. 


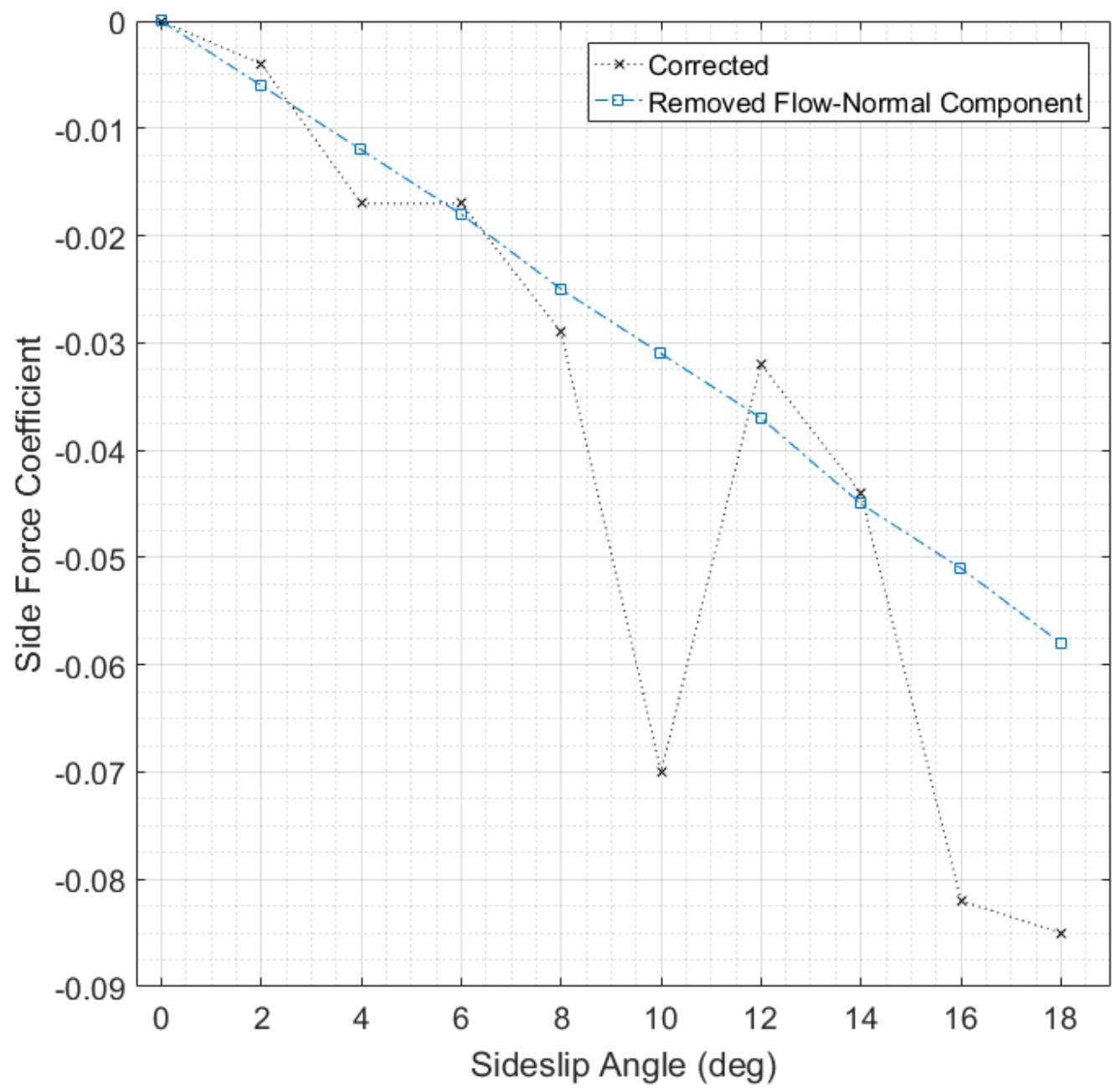

Figure 4.19: Ultra-light model test $-c_{Y}$ vs. $\beta$ (removed flow-normal load cell measurements).

From these corrections, it can be seen that when only considering the effect of the axial load cell, an expected linear trend is seen. Furthermore, when comparing these results to the measured and corrected values, it can be seen that the trends are similar for both results except for the aforementioned sideslip angles at which discrepancies are observed. The key results of the lateral static stability experiments are summarized in the Table 4.4 
Table 4.4: Summary of the results for the static stability derivatives $c_{N_{\beta}}$ and $c_{Y_{\beta}}$ $\left[\mathrm{rad}^{-1}\right]$

\begin{tabular}{|l|l|}
\hline$c_{N_{\beta}}-$ Measured & -0.0274 \\
\hline$c_{N_{\beta}}-$ Removing Flow-Normal Load & -0.0312 \\
\hline$c_{Y_{\beta}}-$ Measured & -0.265 \\
\hline$c_{Y_{\beta}}-$ Calculated from $c_{N}$ & -0.209 \\
\hline$c_{Y_{\beta}}-$ Removing Flow-Normal Load & -0.186 \\
\hline
\end{tabular}

With regards to the second test case, it may have been a possibility that the aircraft's angle of attack could have been affected by the manner in which it is mounted in the wind-tunnel. Fournier tested the impact of the angle of attack on lateral static stability for a highly tapered wing with several body and tail arrangements. It was found that the effect of the angle of attack on both $C_{N_{\beta}}$ and $C_{Y_{\beta}}$ is negligible for $-5^{\circ}$ $<\alpha<5^{\circ}[54$. 


\section{Chapter 5}

\section{Conclusions, Limitations, and}

\section{Recommendations for Future Work}

\subsection{Conclusions}

Wind-tunnel experiments were successfully conducted on an ultra-light wing and ultra-light aircraft. The results of the ultra-light wing test were compared to an existing CFD simulation of this wing. The comparisons indicated that the results of the simulation did not agree with those from experimentation; it is most notably observed in the drag coefficient results as they are clearly erroneous and further work is required on developing a validated simulation. Furthermore, the addition of a boundary layer trip strip on the wing lead to a reduction in lift, an increase in drag, and a delay in the onset of stall.

In regards to the ultra-light model as a whole, the model was found to be statically stable in pitch. The degree of static stability is large when compared to the historical results obtained from other hang glider wings (i.e. the magnitude of $c_{m_{\alpha}}$ is considerably large). In terms of weathercock stability, the wind-tunnel results have shown that the aircraft was not statically stable in yaw. Similar to the wing-only 
tests, the addition of a trip strip on the wing and fuselage delayed the onset of stall. Conversely, a reduction in lift was not observed on the wing and fuselage model due to the trip strip. The height of the trip strip is relatively large when compared to the maximum thickness of the wing. The thickness of the trip strip is commonly less than $1 \%$ of the maximum thickness of the wing. This was not the case for these experiments based on the height selection methodology used. A trip strip with a large thickness will alter the camber of the airfoil leading to entirely different airfoil characteristics. Moreover, the ultra-light model's drag polar did not conform to a conventional aircraft drag polar. The assumption that the parasitic drag does not vary as the angle of attack changes may not be suitable for ultra-light aircraft. Furthermore, the interaction between the fuselage and wing at high angles of attack may also be a factor that has an influence on the performance.

\section{$5.2 \quad$ Limitations}

There were limitations due to ultra-light model, the nature of the external balance, and wind-tunnel that did not allow for a complete static stability and performance analysis. First, dynamic similarity was difficult to achieve due to the size of the model and the maximum attainable speeds in the wind-tunnel. Furthermore, the rigid body model of the wing is not entirely representative of the full scale ultra-light wing due to the flexibility of the wing being neglected. The wing was modeled from a full scale wing in a static ground configuration with no aerodynamic loading. In cruise, the aerodynamic loading will considerably deform the wing to a new static state. In regards to the external balance, it is not possible to resolve the rolling moment stability in yaw of the aircraft given the current configuration of the external balance. Furthermore, the stall characteristics of the aircraft were not attainable due to the physical restrictions of the balance and test section. As the angle of attack increases, 
the model approaches the wind tunnel ceiling. Once the model enters the tunnel's boundary layer, the results cannot be used. Finally, the accuracy of the load cells were not suitable for side-force measurements and the uncertainty of these results were large.

\subsection{Recommendations}

There are improvements and modifications that can be made in order simplify the experiment and to more accurately represent an ultra-light trike. Some suggestions for future work are listed below:

- While taking blockage effects into consideration, 3D printing a larger wing and fuselage would allow for greater Reynolds numbers to be achieved. The results of these experiments could also provide validation of the current results.

- It is recommended that a 3D model of the ultra-light wing be developed in its cruise condition (i.e. when lift $=$ weight and thrust $=$ drag); this would be more representative of real flight conditions.

- Cost permitting, the incorporation of a six-axis load cell would allow for a more complete representation of the aerodynamic loads acting on the aircraft. This would also allow for an analysis on roll moment stability in yaw.

- The location and height of the trip strip were selected from a fundamental methodology that has been fine-tuned in recent years. It is recommended that further tests be undertaken on trip strip placement and height. A sensitivity study may be undertaken to study the effect of trip strip position and height. The purpose of this exercise is to match boundary layer transition as accurately as possible with respect to the full scale wing while reducing the trip strip's effect on the aircraft's performance. 
- It is recommended that an automated data acquisition system be developed. During experimentation, each set of prongs needed to be consecutively inserted into the multimeter to measure the voltage at each angle of attack. Furthermore, the temperature and manometer readings had to be read and recorded manually for each data point. The incorporation of a digital pressure transducer, a digital thermometer, and automated data acquisition system could shorten testing time and increase accuracy.

- Turbulence intensity could have an impact on boundary layer transition and therefore it should be measured with the use of a hot wire anemometer or a cobra probe in the permissable testing location. 


\section{References}

[1] Transport Canada. Ultra-light Aeroplane Transition Strategy. Government of Canada, 1996.

[2] Knauff, T. The Glider Flying Handbook. BookBaby, 1st Edition, 2012.

[3] North Wing (2017). Sport X2 Apache Trike. http://www.northwing.com, accessed March 27, 2017.

[4] Pipistrel Aircraft Canada Inc. (2016). Virus Short Wing. http://www.pipistrel.ca, accessed March 27, 2017.

[5] LAMAC. Design Standards for Advanced Ultra-light Aeroplanes. Light Aircraft Manufacturers Association of Canada, 2001.

[6] Airman Testing Standards Branch United States Department of Transportation, Federal Aviation Administration. Weight-Shift Control Aircraft Flying Handbook. 2008.

[7] Gratton, G.B. The weightshift-controlled microlight aeroplane. Proceedings of the Institution of Mechanical Engineers, Part G: Journal of Aerospace Engineering, 215(3), 2001.

[8] Cook, M.V. A Summary Report on an Experimental Investigation Into Methods for Quantifying Hang Glider Airworthiness Parameters. College of Aeronautics report. Cranfield Institute of Technology, 1987.

[9] Pope, A. Basic Wing and Airfoil Theory. Dover Books on Aeronautical Engineering Series. Dover Publications, 2009.

[10] Anderson, J.D. Fundamentals of Aerodynamics. McGraw-Hill Series in Aeronautical and Aerospace Engineering. McGraw-Hill, 2011.

[11] Etkin, B. and Reid, L.D. Dynamics of flight. Wiley, 1996. 
[12] Nelson, R.C. Flight Stability and Automatic Control. Aerospace Science \& Technology. McGraw-Hill International Editions, 1998.

[13] Raol, J.R. and Singh, J. Flight Mechanics Modeling and Analysis. CRC Press, 1st Edition, 2009.

[14] Trimmer, L.L. and Clark, E.L. Transformation of Axes Systems by Matrix Methods and Application to Wind Tunnel Reduction - Technical Report. United States Air Force, 1963.

[15] Kroo, I.M. Aerodynamics, Aeroelasticity, and Stability of Hang Gliders - Experimental Results. National Aeronautics and Space Administration. NASA TM81269, 1981.

[16] Cook, M.V. and Spottiswoode, M. Modelling the flight dynamics of the hang glider. The Aeronautical Journal, 109(1102), 2005.

[17] Ochi, Y. Modelling of the longitudinal dynamics of a hang glider. AIAA Modeling and Simulation Technologies Conference, 2015.

[18] Cook, M.V. Flight Dynamics Principles. Butterworth-Heinemann, 2nd Edition, 2007.

[19] White, F.M. Fluid Mechanics. McGraw-Hill Series in Mechanical Engineering. McGraw-Hill, 2008.

[20] Barlow, J.B., Rae, W.H., and Pope, A. Low-speed Wind Tunnel Testing. Wiley, 1999.

[21] Goldstein, R.J. Fluid Mechanics Measurements. Taylor and Francis, 2nd Edition, 1996.

[22] Tavoularis, S. Measurement in Fluid Mechanics. Cambridge University Press, 2009.

[23] Willard, R.S. and Kranzler, S.K. Improved Wind Tunnel Data Reduction Procedure - Technical Report. Sight, Sound, and Motion, 1996.

[24] Chatterjee, S. and Hadi, A.S. Regression Analysis by Example. WileyInterscience, 4 edition, 2006.

[25] AEROS Ltd. Trike Wing 'PROFI TL' Manual, Accessed November 2016. 
[26] Stratasys. ABSplus. http://www.stratasys.com/materials/fdm/absplus. Accessed March 2017.

[27] Ahmed, F. Initial CFD Model of Wing - Final Report. Carleton University Department of Mechanical and Aerospace Engineering, 2016.

[28] Katz, J. and Walters, R. Effects of Large Blockage in Wind-tunnel Testing. Journal of Aircraft, Vol. 32, No. 5, pp. 1149-1152, 1995.

[29] Haque, A.U., Asrar, W., Omar, A.A., Sulaeman, E., and Ali, M.J.S. Comparison of Data Correction Methods for Blockage Effects in Semispan Wing Model Testing. EDP Sciences, EPJ Web of Conferences, 114 02129, 2016.

[30] Maskell, E.C. A Theory of the Blockage Effects on Bluff Bodies and Stalled Wings in a Closed Wind Tunnel. Aeronautical Research Council, Reports and Memoranda No. 3400, 1965.

[31] Schlichting, H. and Gersten, K. Boundary-Layer Theory. Springer-Verlag, 2016.

[32] Traub, L.W. Experimental Investigation of the Effect of Trip Strips at Low Reynolds Number. 2011.

[33] Rona, A. and Soueid, H. Boundary Layer Trips for Low Reynolds Number Wind Tunnel Tests. 48th AIAA Aerospace Sciences Meeting Including the New Horizons Forum and Aerospace Exposition, 2010.

[34] Seddighi, M. and Soltani, M.R. Effect of Surface Roughness on an Airfoil in Pitching Motion. 36th AIAA Fluid Dynamics Conference and Exhibit, Fluid Dynamics and Co-located Conferences, 2006.

[35] Choi, D.H. and Lee, E.H. Prediction of Separation Bubbles Using Improved Transition Criterion with Two-Equation Turbulence Model. AIAA Journal, Vol. 33, No. 8, pp. 1512-1514., 1995.

[36] Drela, M. Flight Vehicle Aerodynamics. MIT Press, 1st Edition, 2014.

[37] Currie, I.G. Fundamental Mechanics of Fluids. Marcel Dekker, 1st Edition, 2003.

[38] Raymer, D.P. Aircraft Design: A Conceptual Approach. American Institute of Aeronautics and Astronautics, 5th Edition, 2012.

[39] Fluke. Fluke 175, 177, 179 True-RMS Multimeters - Users Manual (2015), accessed July 7, 2016. 
[40] Down, M., Czubank, F., Gruska, G., Stahley, S., and Benham, D. Measurement System Analysis. Chrysler Group LLC, Ford Motor Company, and General Motors Corporation, 2010.

[41] Kilkenny, E.A. An Experimental Study of the Longitudinal Aerodynamic and Static Stability Characteristics of Hang Gliders. PhD thesis. College of Aeronautics, Cranfield Institute of Technology, 1986.

[42] Jacobs, E.N. and Sherman, A. Airfoil Section Characteristics as Affected by Variations of the Reynolds Number. NACA Report-586, 1937.

[43] Mokhtar, W. A Numerical Parametric Study of High-Lift Low Reynolds Number Airfoils. 2005.

[44] Ananda G.K., Sukumar P.P., and Selig M.S. Measured Aerodynamic Characteristics of Wings at Low Reynolds Numbers. Aerospace Science and Technology, Vol. 42, pp. 392-406, 2015.

[45] Abbott, I.H., von Doenhoff, A.E., and Stivers Jr, L.S. Summary of Airfoil Data. NACA Report-824, 1945.

[46] Kirby, D.A. Low-Speed Wind-Tunnle3 Measurements of the Lift, Drag and Pitching Moment of a Series of Cropped Delta Wings. Aeronautical Research Council, Reports and Memoranda No. 3744, 1974.

[47] Wainfan, B. Wind tunnel - trikes. KITPLANES, pages 98-100, December 2016.

[48] Schmitz, F. Aerodynamics of the Model Airplane. Part I. Airfoil Measurements. 1942.

[49] Roskam, J. Airplane Design. DARcorporation, 1st Edition, 1985.

[50] Anderson, J.D. Introduction to Flight. McGraw-Hill, 3rd Edition, 1989.

[51] Hoerner, S.F. Fluid Dynamic Drag. Published by Author, 1965.

[52] Ormiston, R.A. Wind Tunnel Tests of Four Flexible Wing Ultralight Gliders. National Aeronautics and Space Administration. NASA CP-2085, 1979.

[53] de Matteis, G. Dynamics of Hang Gliders. Journal of Guidance, Control, and Dynamics, Vol. 14, No.6, pp.1145-1152, 1991. 
[54] Fournier, P.G. Low-Speed Investigation of Static Longitudinal and Lateral Stability Characteristics of an Airplane Configuration with a Highly Tapered Wing and Several Body and Tail Arrangements. National Advisory Committee for Aeronautics, NACA RM-L57A08, 1957. 


\section{Appendix A}

\section{Transformation Between Axes Systems}

The following equations describe the transformation of aerodynamic loads from a wind axes $(w)$ system to a body axes system $(b)$ :

$$
\begin{gathered}
L_{b}=n_{w} \\
D_{b}=a_{w} \\
Y_{b}=n_{w} \cos (\beta)-a_{w} \sin (\beta) \\
M_{b}=M_{w} \cos (\beta)+\mathscr{L}_{w} \sin (\beta) \\
\mathscr{L}_{b}=-M_{w} \sin (\beta) \cos (\alpha)+\mathscr{L}_{w} \cos (\beta) \cos (\alpha)-N_{w} \sin (\alpha) \\
N_{b}=-M_{w} \sin (\beta) \sin (\alpha)+\mathscr{L}_{w} \cos (\beta) \sin (\alpha)+N_{w} \cos (\alpha)
\end{gathered}
$$

Transforming the aerodynamic loads from wind axes to stability axes is achieved by setting $\alpha=0$ in the expressions above. 

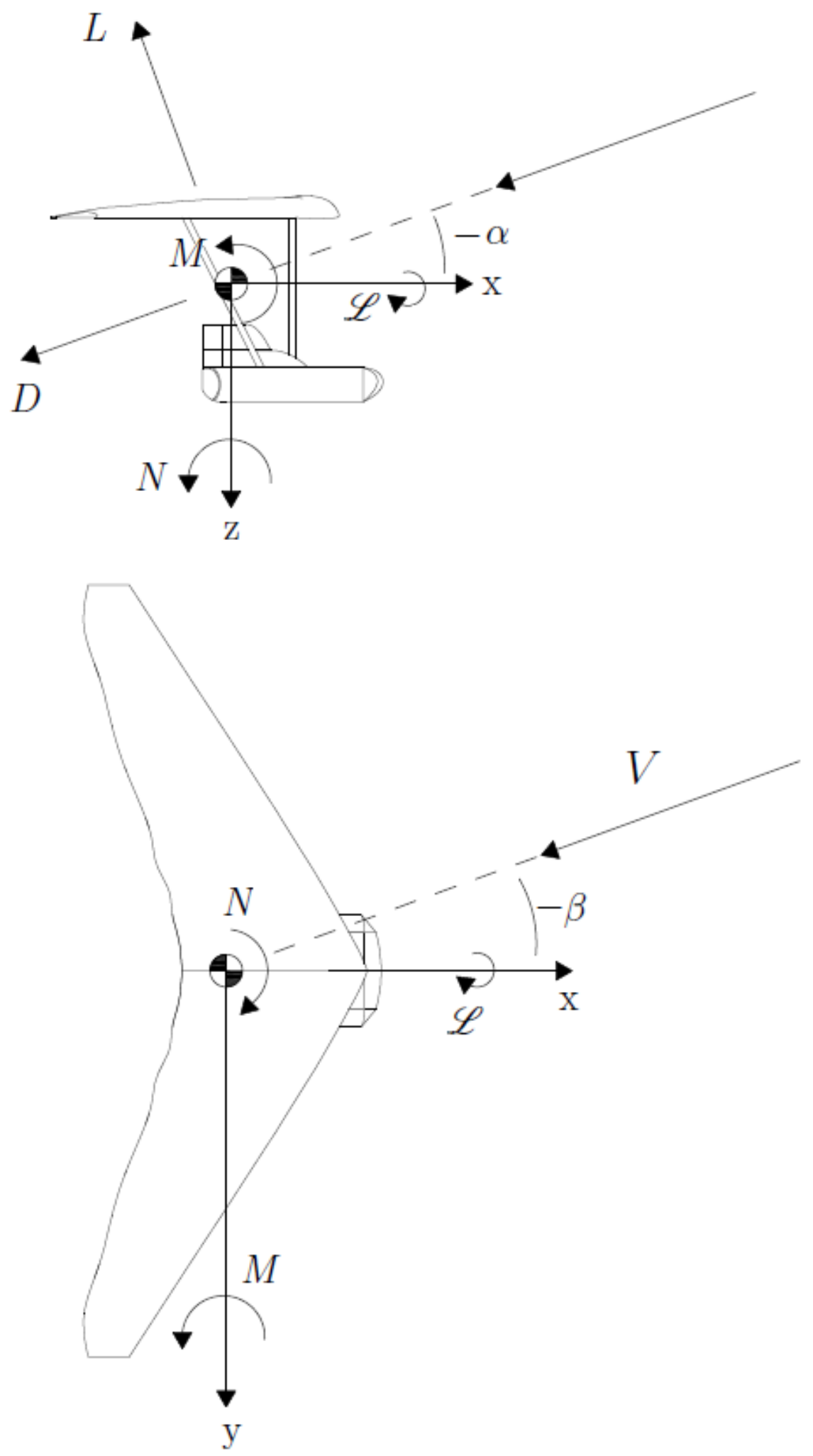

Figure A.1: Ultra-light trike body axes system. 


\title{
Appendix B
}

\section{Calibration Curves for External Balance}

\author{
Calibration Matrix
}

$$
\left[\begin{array}{l}
F_{r, N} \\
F_{r, A} \\
F_{r, M}
\end{array}\right]=\left[\begin{array}{ccc}
1.000 & 0.007 & 0.124 \\
0.002 & 1.000 & -0.001 \\
0.014 & -0.008 & 1.000
\end{array}\right]\left[\begin{array}{l}
F_{a l, N} \\
F_{a l, A} \\
F_{a l, M}
\end{array}\right]
$$

$$
\left[\begin{array}{l}
F_{a l, N} \\
F_{a l, A} \\
F_{a l, M}
\end{array}\right]=\left[\begin{array}{ccc}
1.002 & -0.008 & -0.124 \\
-0.002 & 1.000 & 0.001 \\
-0.014 & 0.008 & 1.002
\end{array}\right]\left[\begin{array}{l}
F_{r, N} \\
F_{r, A} \\
F_{r, M}
\end{array}\right]
$$




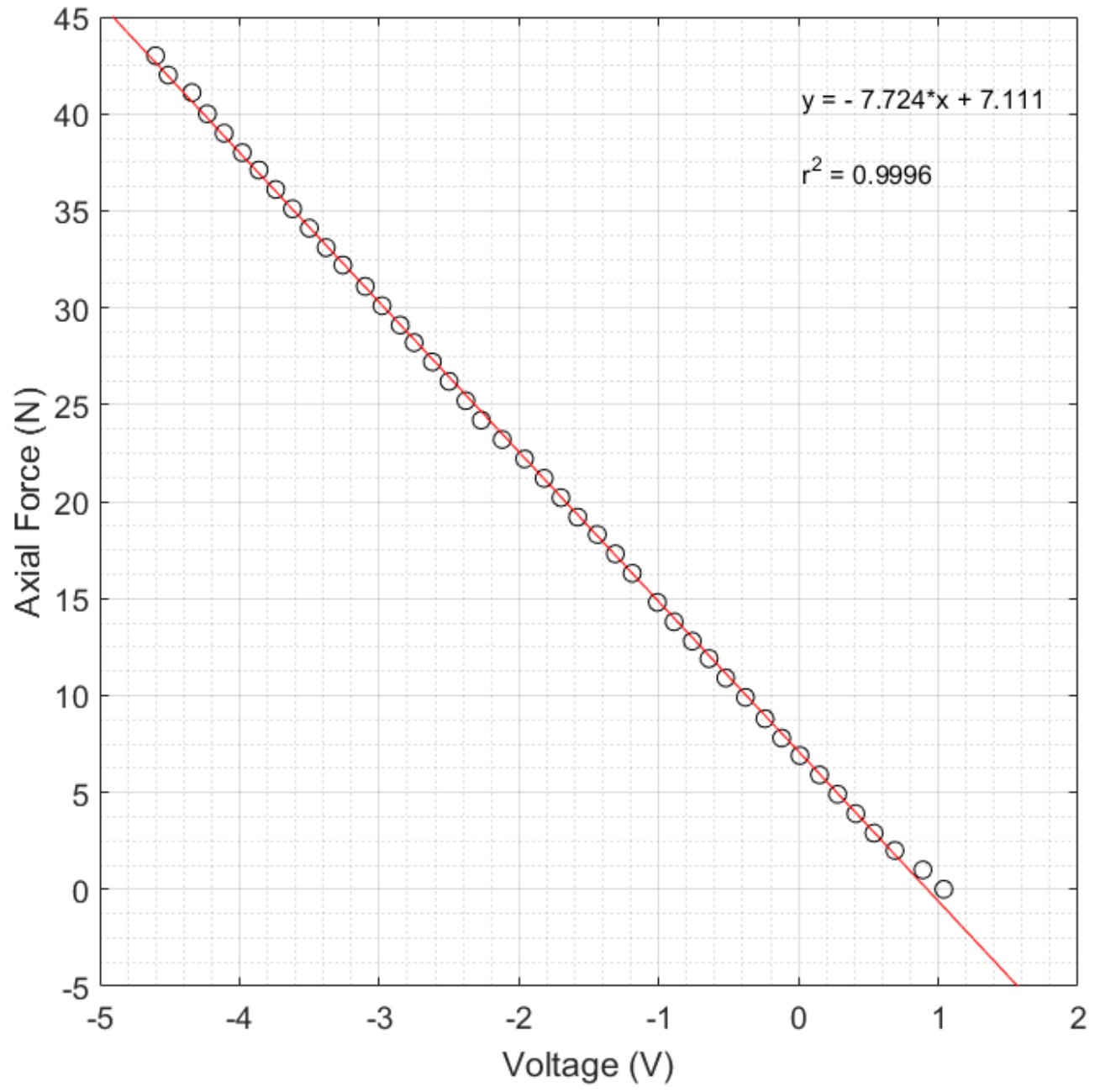

Figure B.1: Axial load cell calibration curve. 


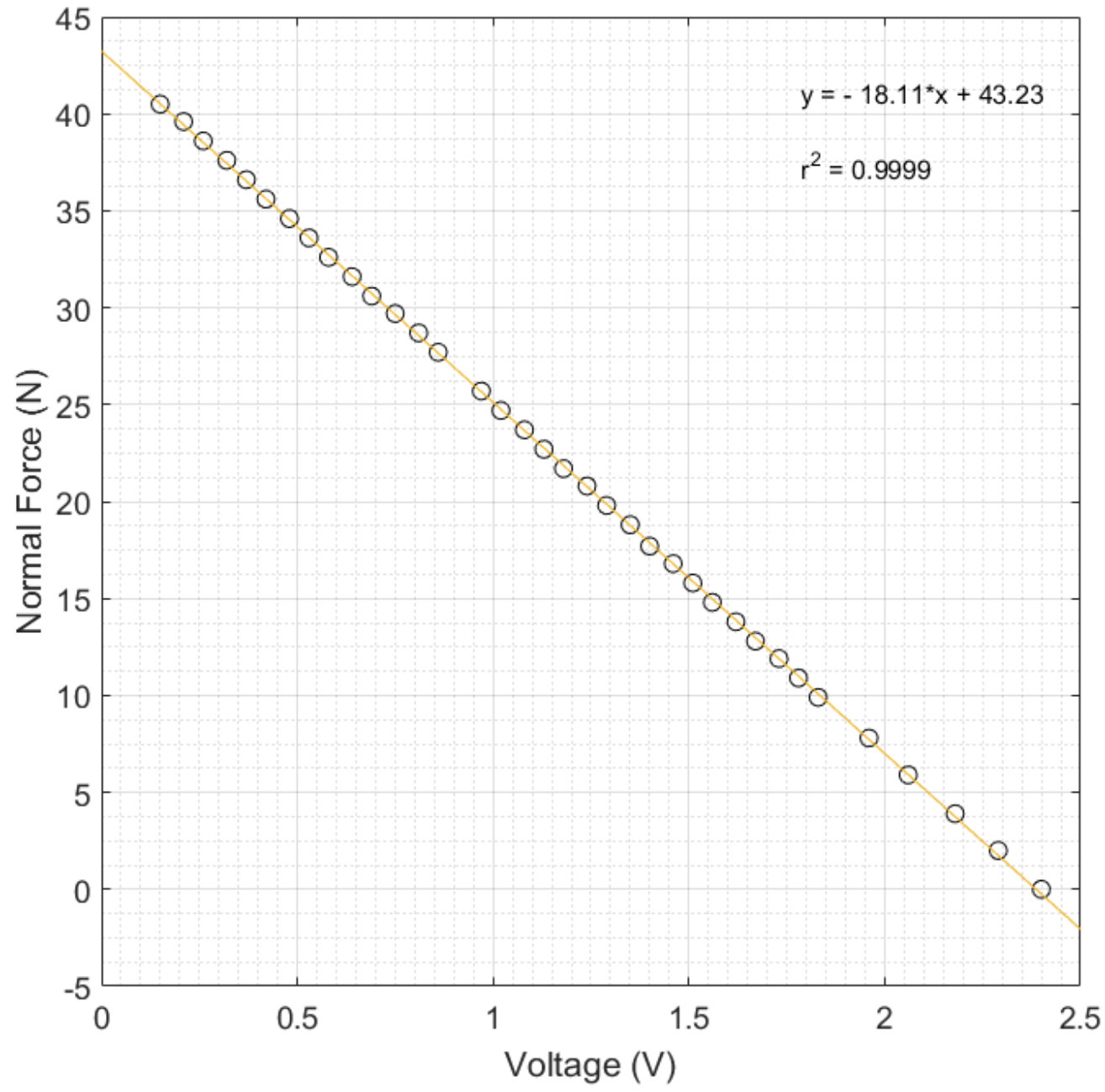

Figure B.2: Normal load cell calibration curve. 


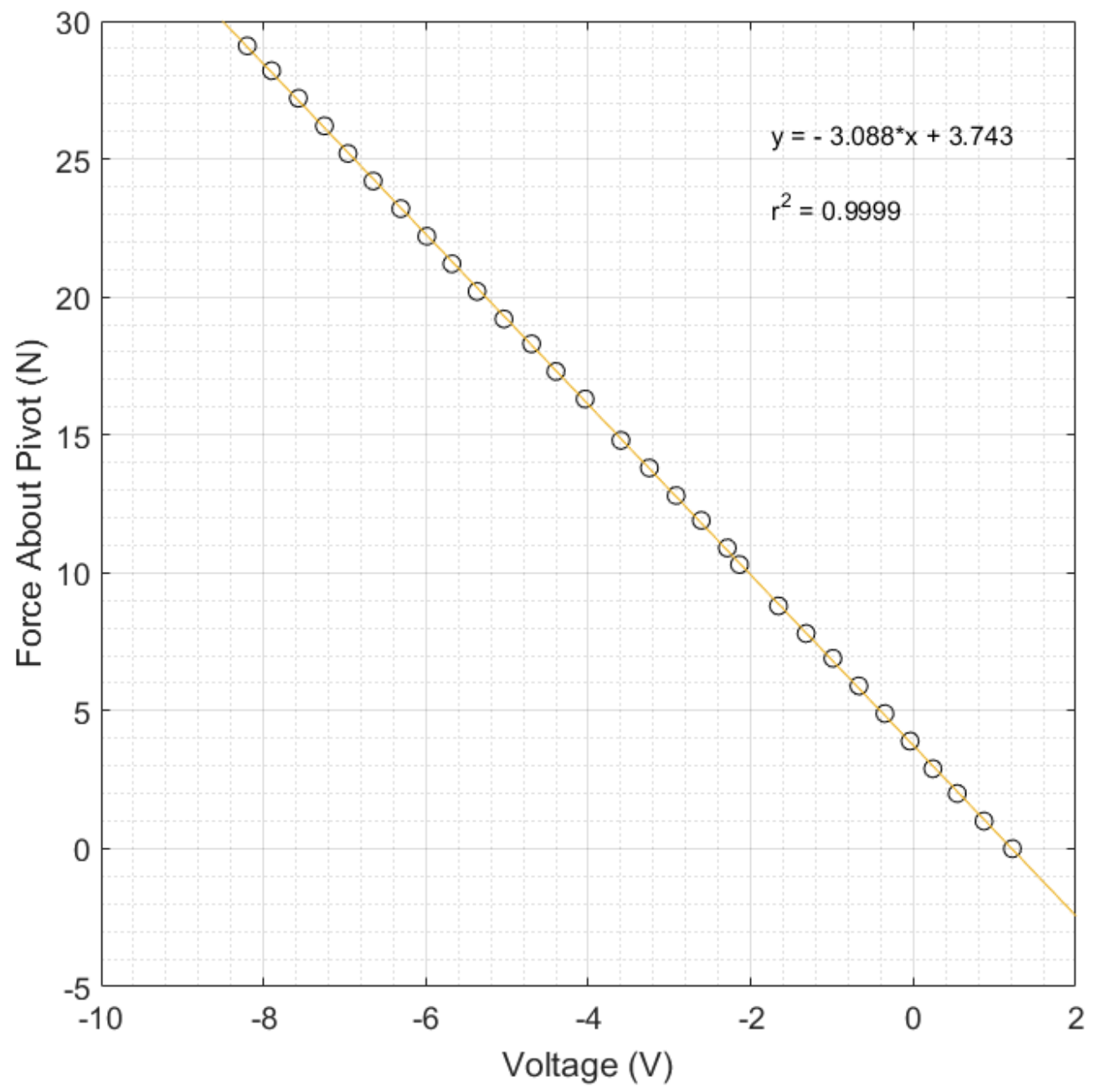

Figure B.3: Pivot load cell calibration curve. 


\section{Appendix $\mathrm{C}$}

\section{Ultra-light Trike Reynolds Number in Various Flight Conditions}

Table C.1: Typical Reynolds Number Range for Ultra-light Trikes

\begin{tabular}{|c|c|}
\hline Flight Condition & Reynolds Number \\
\hline Takeoff & $1.5 \cdot 10^{6}-2.5 \cdot 10^{6}$ \\
\hline Cruise & $3.5 \cdot 10^{6}-4.5 \cdot 10^{6}$ \\
\hline Landing & $2.0 \cdot 10^{6}-3.3 \cdot 10^{6}$ \\
\hline
\end{tabular}

